\title{
Combining local and global smoothing in multivariate density estimation
}

\author{
Adelchi Azzalini \\ University of Padua, Italy
}

15th June 2018

\begin{abstract}
Non-parametric estimation of a multivariate density estimation is tackled via a method which combines traditional local smoothing with a form of global smoothing but without imposing a rigid structure. Simulation work delivers encouraging indications on the effectiveness of the method. An application to density-based clustering illustrates a possible usage.
\end{abstract}

Keywords: non-parametric density estimation; log-linear models; kernel method; densitybased clustering.

\section{Local and global smoothing}

Consider estimation of the probability density function $f(\cdot)$ of a continuous random variable in cases when a parametric formulation for $f$ is not considered appropriate. Given a random sample drawn form $f$, a variety of non-parametric estimation methods are available. Most of these methods share the common feature of being 'fully non-parametric', meaning that the set of competing alternatives from which an estimate $\hat{f}$ must be selected is constituted by the entire set of possible densities, except for some conditions of mathematical regularity.

Limited work has been dedicated to methods which allow inclusion of some qualitative requirement about $f$. One problem which has attracted a fair amount of attention is estimation, in the univariate case, of a unimodal density or, more generally, of a density with a pre-assigned number of modes, like in Hall \& Huang (2002). Other qualitative requirements on $f$ seem to have received less consideration.

We shall be dealing with estimation of a density $f(\cdot)$ on $\mathbb{R}^{d}$, or possibly a subset of it, with $d>1$. For reasons which will become clear shortly, the case $d=2$ is technically possible, but both uninteresting and nearly degenerate in our framework; therefore $d \geq 3$ is the situation really considered.

It is well-known that, as $d$ increases, non-parametric methods, and in particular those for density estimation, degrade in performance, eventually running into the so-called problem of 'curse of dimensionality' when $d$ is large. On the other hand, there is the widespread perception that, in many real situations, the dependence structure of a multivariate distribution is largely governed by the dependence among a smaller number of components. An explicit statement of this view has been expressed by Scott (1992, p.195): "Multivariate data in $\mathbb{R}^{d}$ are almost never $d$-dimensional. That is, the underlying structure of data in $\mathbb{R}^{d}$ is almost always of dimension lower than $d$ ". 
The present contribution examines an estimation method motivated by these considerations. Broadly speaking, we impose a 'light structure' on the density $f(\cdot)$, moving away from a fully non-parametric construction, but without imposing a detailed structure, such as parametric form. A bit more specifically, it is assumed that, at least in an approximate sense, the dependence is regulated by a structure based on $m$-dimensional subsets of the variables, with $m<d$. The introduction of this constraint leads to a form of global smoothing of the estimated distribution which can improve upon existing methods, in appropriate situations, by reducing variability connected to estimation of fine details of $f(\cdot)$ regulating high-order interactions among variables. It is plausible that, even if these high-order interactions are not exactly null, the reduction of variability of the estimate overcomes the bias so introduced; an assumption of this sort is ubiquitous in any modelling operation.

Clearly, the success of this scheme relies on the suitability of the imposed structure in a given situation. To exemplify by what here represents an extreme case, application of the stated criterion when $d=2$ would entail to introduce a joint distribution constituted only by marginals of dimension $m=1$, that is, assuming independence of the two component variables. In the majority of situations, the more interesting ones in fact, this extreme simplification would not be appropriate; this explains why earlier we have effectively restricted ourselves to the case $d \geq 3$.

Estimation of $f(\cdot)$ will still be carried out via a classical local smoothing method, such as the kernel estimator, but in a way which reflects the global smoothing imposed by the assumed structure of $f(\cdot)$. Therefore the final outcome of the procedure will reflect both the local and the global smoothing operations.

In the next two sections, we transfer this broad criterion into a specific operational formulation. This is then followed by numerical exploration to evaluate its practical working with simulated data and by its utilization within a density-based clustering process of some real data.

\section{Global smoothing via a log-linear model}

The criterion described only qualitatively so far can be translated into an operational procedure. Given the broad nature of the above formulation, there is not a unique prescribed way to define such a procedure. The route to be presented here is driven by simplicity and flexibility, since it can be used in conjunction with any local smoother which allows weighted observations with only simple adjustments of an existing method.

Assume that a sample $z_{1}, \ldots, z_{n}$ of observations drawn from $f(\cdot)$ is available, where $z_{i}=$ $\left(z_{i 1}, \ldots, z_{i d}\right)^{\top}$ for $i=1, \ldots, n$. Denote by $Z=\left(Z_{1}, \ldots, Z_{d}\right)^{\top}$ the parent random variable from which the $z_{i}$ 's are drawn, all independently from each other. We introduce subdivisions of the $d$ coordinates axes into disjoint $r_{1}, \ldots, r_{d}$ intervals, creating a partition of the sample space into $r=r_{1} \times \cdots \times r_{d}$ hyper-rectangles or cells. Correspondingly, there are probabilities $\pi_{1}, \ldots, \pi_{r}$ associated to the cells. The $j$ th element of this partition, denoted $R_{j}$, can be associated to a multidimensional subscript $j_{1}, j_{2}, \ldots, j_{d}$, but this is not of relevance at the moment.

Denote by $n_{j}$ the number of sample elements falling into $R_{j}$, for $j=1, \ldots, r$, so that $\sum_{j} n_{j}=n$. The basic estimate of $\pi_{j}=\mathbb{P}\left\{R_{j}\right\}$ is $n_{j} / n$ and, correspondingly, for a given point $x \in R_{j}$, a crude estimate of $f(x)$ is

$$
\frac{n_{j}}{n \operatorname{vol}\left(R_{j}\right)}
$$


where $\operatorname{vol}\left(R_{j}\right)$ is the geometric volume of $R_{j}$.

A key weakness of this scheme is that it implies $r-1$ distinct probabilities $\pi_{j}$ to be estimated, up to a constraint on their sum. If $d$ is not small and the $r_{1}, \ldots, r_{d}$ subdivisions are not coarse, $r$ can be large. To reduce the number of free parameters to be estimated, we introduce a log-linear model for the cells probabilities, $\pi_{j}$ 's, where interaction terms involving more than $m$ component variables are set to zero. For instance, if $d=3$ and we choose $m=2$, terms of the log-linear representation of the $\pi_{j}$ 's are retained up to pairwise interactions while all three-factor interaction terms are eliminated, reducing the number of underlying parameters by $\left(r_{1}-1\right)\left(r_{2}-1\right)\left(r_{3}-1\right)$ with respect to the saturated model. See Section 9.2.2 of Agresti (2013) for a detailed discussion of the pertaining log-linear model; there is only the difference that those expressions refer to the expected values of the frequencies instead of the probabilities, but this is irrelevant since the two sets of quantities are proportional to each other.

After the log-linear model has been fit to the observations, a set of expected frequencies is obtained, denoted $\hat{n}_{j}$, and corresponding estimated probabilities $\hat{\pi}_{j}(j=1, \ldots, s)$. Replacing $n_{j}$ by $\hat{n}_{j}$ in (11) provides a revised estimate.

The constraints enforced by the log-linear model refer to the cell probabilities, $\pi_{j}$, and so to the expected frequencies $\hat{n}_{j}$, but not to the density $f(x)$. However, provided the values $\operatorname{vol}\left(R_{j}\right)$ are not too disparate, at least in the region where most of distribution is located, the originally intended dependence structure will hold approximately. Furthermore, the local smoothing step to be presented shortly introduces an additional perturbation in this sense. Since the imposed dependence structure is motivated by practical considerations of improved estimation performance rather than exact model compliance, we are not concerned about these approximations.

\section{Non-parametric local smoothing}

In the second step of the procedure, the expected frequencies $\hat{n}_{j}$ obtained in the first step are used to assign weights to the observations, $z_{i}$, so that the estimate $\hat{f}$ produced by the subsequent local smoothing respects, approximately, the estimated cell probabilities $\hat{\pi}_{j}$.

To illustrate the procedure, we use the following simple form of the kernel density estimate. Take the kernel function to be the $d$-dimensional circular normal density with standardized components, denoted $\varphi_{d}(\cdot)$, and choose a vector $h_{1}, \ldots, h_{d}$ of smoothing parameters; then the classical kernel estimate at point $x \in \mathbb{R}^{d}$ is

$$
\frac{1}{n \operatorname{det}(h)} \sum_{i=1}^{n} \varphi_{d}\left(h^{-1}\left(x-z_{i}\right)\right)
$$

where $h=\operatorname{diag}\left(h_{1}, \ldots, h_{d}\right)$. This classical estimate is modified by weighting an observation in the $j$-th cell with $w_{j}=\hat{n}_{j} / n_{j}$, so that the overall weight of the $n_{j}$ observations in the $j$ th cell is $\hat{n}_{j}$ instead of $n_{j}$. The new estimate takes the form

$$
\hat{f}(x)=\frac{1}{n \operatorname{det}(h)} \sum_{i=1}^{n} w_{j(i)} \varphi_{d}\left(h^{-1}\left(x-z_{i}\right)\right)
$$

where $w_{j(i)}$ is the weight of the cell to which $z_{i}$ belongs. The type of perturbation of estimator (2) is denoted 'tilting' by Doosti \& Hall (2016).

Clearly several variants forms can be considered, such as replacing the normal kernel in (3) by some other multivariate kernel or using smoothing parameters which vary with the 
observations. Not only these variants are immediately accommodated, but we are not restricted to kernel-based methods. For instance, if we use instead a projection method based on an orthogonal series decomposition of $f$, the weight $w_{j(i)}$ is assigned to observation $z_{i}$ when the coefficients of the projection are estimated as sample averages of suitable data-dependent functions.

A complication arises with empty cells, where $n_{j}=0$, since the corresponding weights $w_{j}$ are not well defined. While for cells with $n_{j}>0$ the method works by suitably increasing or decreasing the weight of the observations belonging to that cell, no such adjustment is possible if the cell is empty.

The simplest approach to the problem is to just use (3), only replacing $n$ in the denominator by $n_{w}=\sum_{i} w_{j(i)}$; we are then effectively ignoring the 'empty cells' problem. This variant form is denoted 'Plain' (or P) later on. When $\hat{n}_{j}$ corresponding to $n_{j}=0$ is of non-negligible magnitude, possibly so for several cells, the above solution may appear too crude, hence supporting the alternative approach of introducing some fictitious data to fill the empty cells. Note that this implies that the overall number of data points exceeds $n$, although the sum of their weights remains $n$. However, while appealing in principle, it is hard to say how to pursue this route in a theoretically-motivated procedure. An heuristic method has been constructed, described in Appendix A. This variant form is denoted 'Fill' (or F) later on.

\section{Practical and computational aspects}

The practical implementation of the method requires to specify, in the first place, the subdivisions of the axes which identify the $r=r_{1} \times \cdots \times r_{d}$ cells. For each axis, we have started by applying the 'normal reference rule' proposed by Scott $(1992$, p. 82) for choosing the histogram bins, assuming joint normality of the multivariate distribution, that is, the $k$ th bin-width is initially taken equal to

$$
b_{k} \approx 3.5 \sigma_{k} n^{-1 /(2+d)}
$$

where $\sigma_{k}$ is the standard deviation of the $k$-th component variable, $Z_{k}$; in practice, $\sigma_{k}$ must be replaced by its sample value. Division of the range of observations $\left(z_{1 k}, \ldots, z_{n k}\right)$ by $b_{k}$, rounded to the nearest integer, lends the number of bins for the $k$-th component, $r_{k}$. The sample range of the $k$-th variable has then been subdivided into $r_{k}$ intervals, constructed as follows: first the quantiles of level $\left(0,1 / r_{k}, \ldots, r_{k-1} / r_{k}, 1\right)$ of the $\operatorname{Beta}(3 / 4,3 / 4)$ distribution have been computed and then the sample quantiles with level equal to these Beta quantiles have been used as the end-points of the intervals on the $k$-th axis. The underlying idea is to have the central intervals shorter than those near the margins of the sample range. This process is repeated for $k=1, \ldots, d$.

This procedure for choosing the end-points of the intervals appears somewhat arbitrary if examined from a formal viewpoint. The scheme must rather be regarded as a way of mimicking the non-automatic process followed when intervals are chosen by subjective judgement.

To compensate the possible effect of the choice of the number of subdivisions $r_{1}, \ldots, r_{d}$, a variant form of the procedure involved using three choices of the subdivisions: one as described above, one decreasing each $r_{k}$ by 1 and the third one increasing each $r_{k}$ by 1 . For each of these three grids, the log-linear fitting and computation of (3) were applied, followed by averaging of the three estimates. This variant is denoted 'Average' (or A) later on.

Once the grid of the $\mathbb{R}^{d}$ space has been fixed, the sample frequencies $n_{j}$ of the cells are identified. For a given value of $m$, we must fit a log-linear model as described in Section 2 , 


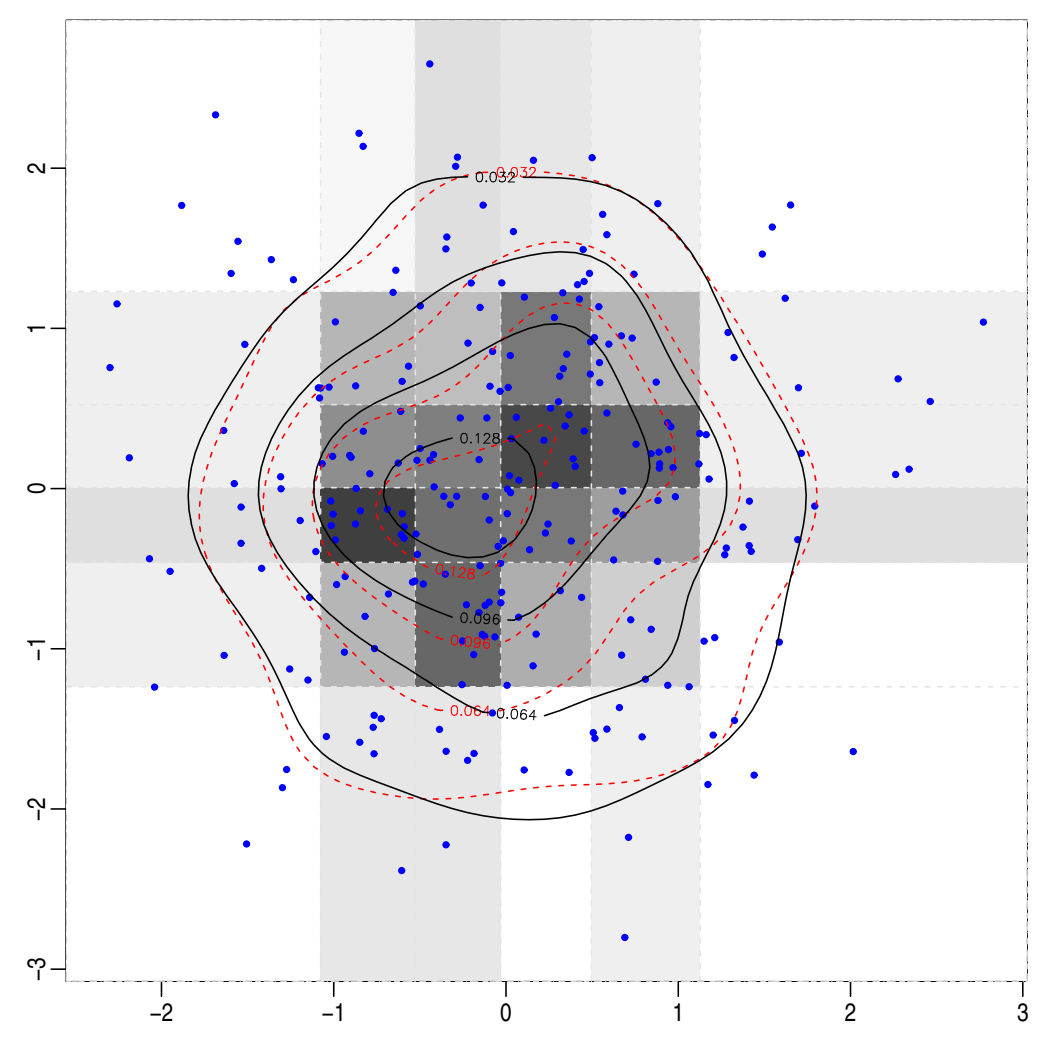

Figure 1: Illustrative example of the estimate in the simple case with $d=2$ and $m=1$

we temporarily leave aside the choice of $m$, to which we return later. In our problem the interest is only in the fitted frequencies, $\hat{n}_{j}$, not in the log-linear parameters. In this case the recommendation of Agresti $(2013, \S 9.7 .3)$ is to adopt the iterative proportional fitting algorithm, since it "converges to the ML [maximum likelihood] fit even when the likelihood is poorly behaved". A Fortran implementation of this algorithm has been provided by Haberman (1972), subsequently ported to the R computing environment with name loglin.

The final step is application of the the weighted estimator (3). In most of our numerical work, the diagonal smoothing matrix $h$ has been chosen by the multivariate version of the plug-in method of Wand and Jones (1994) available in the R package ks (Duong, 2015).

An illustration of the working of the procedure is provided in Figure 1, which refers to the simplest possible case, that is, with $d=2$ and $m=1$. As already explained, this situation is not of practical relevance, but it is appropriate for simple illustration. Specifically, the $n=250$ plotted points constitute a sample drawn from a circular bivariate normal distribution with standardized marginals. A rectangular area slightly wider than the range of the observed points has been selected and, using the above-described rule, a $6 \times 6$ grid has been identified; for all cells of this grid, $n_{j}>0$ was observed. The 36 rectangles have been shaded using a 25-level grey scale which discretizes the values of the crude estimate (11). The dashed red lines represent the contour level curves of the classical kernel estimate (2) while the continuous black curves refer to the weighted estimate (3); this estimate appears somewhat smoother than the unweighted one, with more limited departures from convexity, especially so in the central region. 


\section{Simulation work}

The performance of the proposed method has been examined in a number of cases, using simulated data from a range of distributions: normal, skew-normal, Student's $t$ and its skew version, and two-component mixtures of these distributions. The general expression of the distribution in use is

$$
f(x)=f(x)=\pi f_{1}(x)+(1-\pi) f_{2}(x), \quad x \in \mathbb{R}^{d},
$$

where $f_{1}$ and $f_{2}$ are of skew-normal (SN) or skew- $t$ (ST) type, which include the classical normal and $t$ distributions as special cases; $\pi \in(0,1]$ is the mixing proportion. The distributions $f_{1}$ and $f_{2}$ are specified by the following parameters: a $d$-vector location $\xi$, a $d \times d$ symmetric positive-definite scale matrix $\Omega$, a $d$-vector slant $\alpha$ and a positive real number $\nu$. The component $\nu$ exists only for the ST distribution; when $\nu=\infty$, or equivalently when it is not present, the distribution is of SN type. A detailed treatment of the multivariate SN and ST distributions is provided by Azzalini \& Capitanio (2014). When $\pi=1$, there is effectively no mixture mechanism and only the $f_{1}$ parameters are required. The parameters considered have been selected among the following options.

- If $\pi=1$, the location parameter is always $\xi=(0, \ldots, 0)^{\top}$. If $0<\pi<1$, the location of $f_{1}$ is $2 \times 1_{d}$ and the one of $f_{2}$ is $-2 \times 1_{d}$, where $1_{d}$ denotes the $d$-vector of all 1's.

- The scale matrix $\Omega$ has been chosen among the following options:

- the identity matrix $I_{d}$;

- a Toeplitz-type matrix with $(i, j)$-th entry $\rho^{|i-j|}$ where $\rho=3 / 4$, or equivalently with $\operatorname{AR}(1)$ correlation structure;

- an $\operatorname{ARMA}(2,1)$ correlation structure;

- a matrix with elements specified individually, in some instances with $d=3$.

- The components of the slant parameter $\alpha$ have been specified individually. When $\alpha=0$ and $\pi=1$, the distribution is a regular (symmetric) Gaussian or Student's $t$ distribution.

- The degrees of freedom $\nu$ was given a value among the following: $\infty$ (corresponding to the SN distribution), 5 or 2.

Distributions with dimension from $d=3$ to $d=5$ have been considered. The value $m$ employed in the log-linear model was $m=2$ and $m=3$, with the constraint $m<d$. In most cases, the sample size was $n=500$; a few experiments used either $n=250$ or $n=1000$. For each combination of parameter values and sample size, 2500 replicates have been considered and the following estimation methods have been tested:

1. the classical kernel method in (2), denoted 'kde';

2. the weighted kernel method in (3), in the Plain variant form described in Section 3 ,

3. the Average variant which averages three estimates computed from three grid subdivisions of the sample space;

4. the Fill variant which places constructed points in empty cells, as described at the end of Section 3 and more in detail in an Appendix. 
The vector of smoothing parameters for the kernel method and its variants was obtained by the function Hpi.diag of the R package ks described earlier.

The possible number of factor combinations so obtainable is enormous even if one selects only a few possible choices for each of the above-described parameters. Moreover the computation burden with certain parameters, especially for the Average variant, was appreciable. This prevents any attempt of running a full factorial experiment. Only a selection of factor combinations has been considered, driven by subjective judgement on the outcome of earlier experiments, paying more attentions to situations which appeared more interesting in some sense. For instance, for a certain combination of parameters, the value of $n$ could have been decreased to 250 or increased to 1000 to examine the effect of sample size alone, when this appeared to be an interesting case.

To evaluate the performance of the proposed estimate (3), in their variant forms, with respect to the classical estimate (2), the estimation error at $x \in \mathbb{R}^{d}$ has been expressed by

$$
e(x)=\frac{|f(x)-\hat{f}(x)|}{f(x)^{p}}
$$

where $p=1 / 2$ has been used in the outcomes presented below. Initial numerical work had also considered $p=0$ and $p=1$, but the general qualitative indication which emerged was not very different and $p=1 / 2$ may represent a reasonable compromise between absolute error and relative error. Two sets of points have been considered for evaluating (5): (i) a non-random grid of points spanning the area of non-negligible density of $f(x)$ and (ii) the sample values. The second option is relevant in certain applications like the one of Section 6 . A detailed description of the non-random grid of points is provided in Appendix B. For the (ii) case, only the real observations have been considered in the Fill variant, ignoring the fictitious observations which it involves.

Direct consideration of (5) for all the evaluation points, in either of the two considered sets, is not feasible. The quantiles of such sets of estimation errors have examined instead, at probability levels $(0.25,0.50,0.75,0.90,0.95,0.99)$. Even with this reduction, the amount of tabular material so produced is considerable; the full set of such tables is provided in Appendix B. A more compact summary exhibit of the overall outcome is provided by Figure 2, The values on the vertical axis represent

$$
R(p)=\frac{Q_{0}(p)-Q(p)}{Q_{0}(p)}
$$

where $Q(p)$ is the $p$-level quantile of the relative error (5), evaluated over a given set of points, for a the proposed method (in one of its variants) and $Q_{0}(p)$ is the similar quantity for the standard kernel density estimation. Therefore, $R(p)$ represents a measure of reduction of the estimation error with respect to the classical estimate, or a measure of its increase in case this quantity is negative. Figure 2 reports only the more noteworthy aspects of the full outcome, as described next.

Only variants Plain and Fill have been considered in Figure 2 since the Average form was essentially equivalent to the Plain one, with extra computing effort; it is however reassuring to know that the specific choice of the grid size is not critical. For each of the three panels of the figure, the left portion refers to the choice $m=2$ for the log-linear model, the right portion to $m=3$. Three values of $p$ entering (6) have been reported, namely $p=0.50,0.75,0.95$, from the full six values in the complete outcome. For each pair of $p$ and $m$, there are two vertical stripes of numbers; the left blue stripe refers to distributions which are mixtures, while the right red stripe refers to single-component distributions, that is, those having $\pi=1$ in (4). 

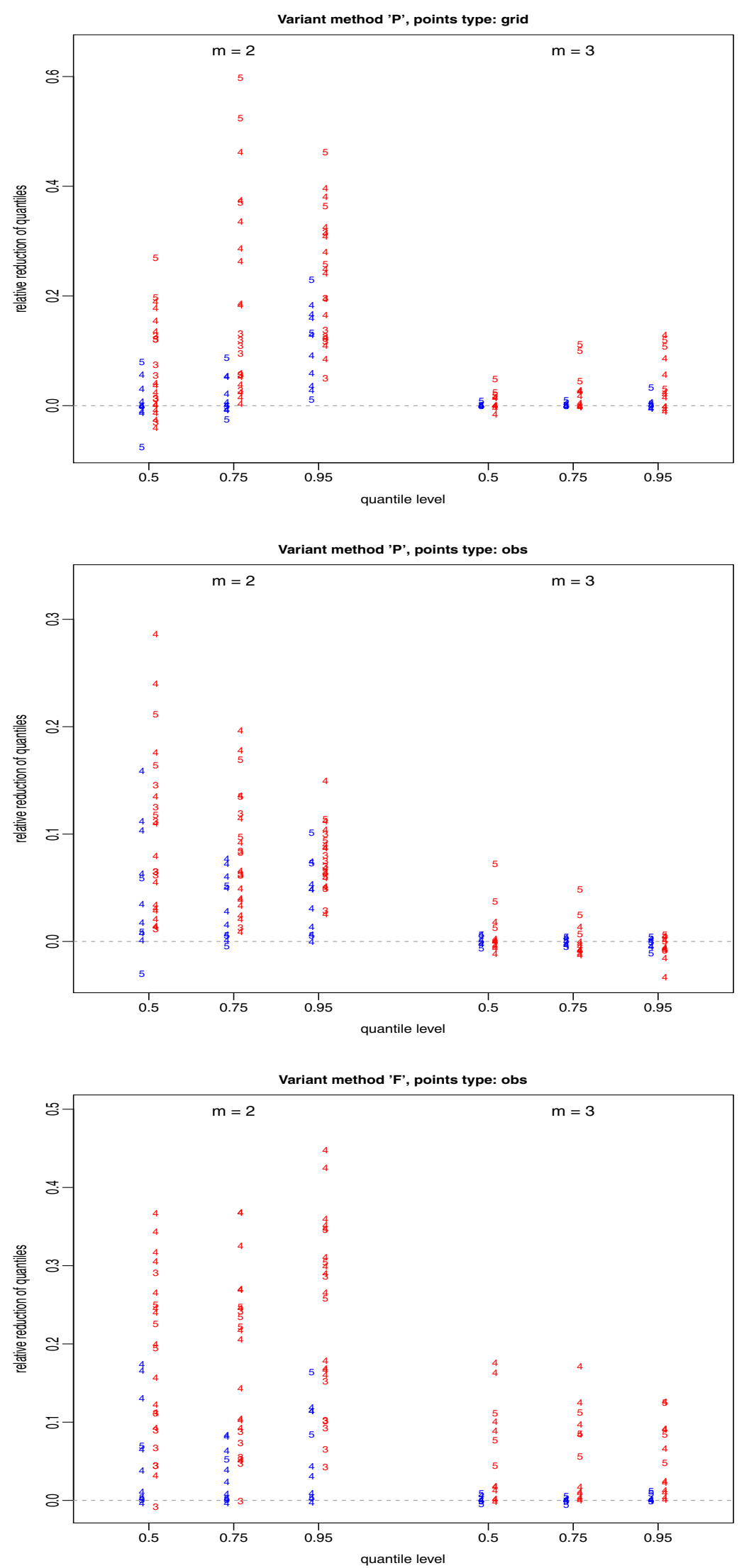

Figure 2: Relative reduction of estimation error quantiles of the proposed estimate versus the classical kernel estimate; see text for a detailed description. 
In all cases, the digit plotted at ordinate (6) denotes $d$. Of the three panels, two refer to the Plain variant of the method, with evaluation is performed either at a fixed grid of points or at the sample points. The third panel refers to the Fill variant, but only with evaluation at the sample points; evaluation at the grid points was markedly unsatisfactory.

The first message emerging from inspection of Figure 2 is that an improvement of the weighted kernel estimate over the classical one occurs in the majority of cases, often with an appreciable magnitude; the negative values are limited in number and in magnitude. This consideration is substantially reinforced if we confine attention to $m=2$, irrespectively of $d$; this explain why $m>3$ has not been considered in the simulations. Another indication is that the method, in all variants, performs better with a single component distribution than with a non-generate mixture.

Operationally, the following recommendations for use of the method can be extracted: (i) set $m=2$ in all cases; (ii) the Plain variant is preferable when the whole density surface must be estimated, while the Fill variant is preferable for evaluation at the observed data points; (iii) expect more improvement in case of a unimodal distribution than a multimodal one. These recommendations refer to the kernel estimate and smoothing matrix described above, and they may not necessarily hold for other forms of non-parametric estimation.

\section{Application to density-based clustering}

The proposed density estimate has been used in conjunction with the clustering method presented by Azzalini and Torelli (2007), implemented in the R package pdfCluster (Azzalini and Menardi, 2014). Since this clustering technique is firmly based on estimation of the density of the underlying $d$-dimensional random variable, it represents an ideal framework for application of the present proposal.

The real-data application presented by Azzalini and Torelli (2007, Section 4.3) concerned eight chemical components of $n=572$ specimens of olive-oil originating from various regions of Italy. We re-examine their clustering exercise whose aim was the reconstruction the production area of the specimens from the values of their chemical components. The data themselves are available in the pdfCluster package. A more detailed description of the data and of their preliminary transformations, which we also apply here, is provided by Azzalini and Torelli (2007). We only specify the undocumented detail that, in the additive log-ratio transform applied to the compositional data $p_{j}(j=1, \ldots, 8)$, namely $y_{j}=\log \left(p_{j} / p_{k}\right)$ for $j \neq k$, the choice $k=4$ was made because the values of $p_{4}$ are well separated from 0 ; this is also the choice of the original article. The first $d=5$ principal components of the $y_{j}$ 's constitute the variables used for the actual clustering step.

The pdfCluster package was applied to the five principal components just described both in its current public version (1.0-2, as available at the time of writing) and a modified version which replaces the classical kernel estimate (2) and the weighted kernel estimate (3) with $m=2$; all other ingredients have been kept at the default specification of the package. Table 1 displays the cross-classification table of the true geographical areas and the groups formed by clustering for the classical estimate (2) in the first three columns and the new proposed estimate in the last three columns refer. The latter estimate has actually been computed using both the Fill and the Plain variant, but the outcome was the same. The ARI values underneath each sub-table denote the 'adjusted Rand index' which constitutes a measure of agreement between the true and the reconstructed classification (Huber and Arabie, 1985). There is a clear improvement in using the new estimate, from consideration both of direct inspection of the table and by the ARI values. The left portion of Table 1 is 
slightly different from the table originally obtained by Azzalini and Torelli (2007), but the essential traits are the same and the ARI value was even smaller there, namely 0.792.

Table 1: Clustering of olive-oil data: true versus reconstructed groups using the current package pdfCluster and its modification with density estimation replaced by the new method.

\begin{tabular}{lrrrrrr}
\hline & \multicolumn{3}{c}{ classical estimate } & \multicolumn{3}{c}{ proposed estimate } \\
& 1 & 2 & 3 & 1 & 2 & 3 \\
\hline South & 321 & 0 & 2 & 323 & 0 & 0 \\
Sardinia & 0 & 98 & 0 & 0 & 98 & 0 \\
Centre-North & 0 & 45 & 106 & 0 & 22 & 129 \\
\hline ARI & & 0.873 & & & 0.937 &
\end{tabular}

The values in Table 1 have been obtained using the default smoothing parameter $h$ of pdfCluster, which is the asymptotically optimal bandwidth under normality, multiplied by a shrinkage factor. For completeness, we considered also the choice of $h$ produced by Hpi.diag, already used in the simulation work. In this case the shrinkage factor usually introduced by pdfCluster has been to the neutral value of 1 , since that shrinkage loses meaning with another choice of $h$. The ARI value of the new groupings decreases slightly to 0.910 for the proposed estimate, while where was a much worse degrade for the classical estimate, which lead to four groups instead of three, with an ARI of 0.817.

At first sight, it may look surprising that the use of estimate (3) produces such a noticeable improvement over (2), considering that Figure 2 indicates a limited improvement in connection with multimodal densities, which is the typical situation in a clustering context. One must however bear in mind that the procedure underlying pdfCluster involves two main stages: in the first stage, the density of the overall population is estimated, to locate the cluster cores associated to the subpopulations, while, in the second stage, the distribution of each identified cluster core is estimated separately. The densities of these sub-populations are naturally of unimodal type, where Figure 2 indicates a better performance. It is then reasonable to link the successful effect of the new estimate mainly to its role in the second stage of the procedure.

\section{$7 \quad$ Final remarks}

The numerical outcome, both from the simulation work and from the clustering application, provides quite clear evidence in support of the proposed method. However, there is still much room for improvement. For instance, a better motivated method for filling empty cells would be welcome. Even more importantly, some mathematically-argumented understanding of why the method works is lacking. Moreover, the global smoothing technique of Section 2 represents one possible route to implement the qualitative criterion stated in Section 1, but other routes may be considered.

An implementation of the proposed method will be made publicly available in the $\mathrm{R}$ package pdfCluster.

Acknowledgements The development of this work has much benefited from stimulating discussions with Giuliana Regoli. 


\section{References}

Agresti, A. (2013). Categorical Data Analysis, 3rd edition. Wiley, New York.

Azzalini, A. with the collaboration of Capitanio, A. (2014). The Skew-Normal and Related Families. Cambridge University Press, IMS monographs.

Azzalini, A. and Menardi, G. (2014). Clustering via nonparametric density estimation: The R package pdfCluster. J. Stat. Software, 57(11), 1-26. http://www.jstatsoft.org/v57/i11/

Azzalini, A and Torelli, N. (2007). Clustering via nonparametric density estimation. Stat. Comput., 17, 71-80.

Doosti, H. and Hall, P. (2016) Making a non-parametric density estimator more attractive, and more accurate, by data perturbation. J. R. Stat. Soc, series B, 78, 445-462.

Haberman, S. J. (1972). Algorithm AS 51: Log-linear fit for contingency tables. Appl. Stat., 21, 218-225.

Hall, P. and Huang, L. S. (2002). Unimodal density estimation using kernel methods. Stat. Sinica, 12, 965-990.

Scott D. (1992). Multivariate Density Estimation. John Wiley \& Sons, New York.

Duong, T. (2015). ks: Kernel Smoothing. R package version 1.10.0. https://CRAN.R-project.org/package=

Wand, M. P. and Jones, M. C. (1994). Multivariate plugin bandwidth selection. Comp. Stat. 9, $97-116$. 


\section{Appendix}

\section{A. Filling empty cells}

As explained in Section 3, empty cells having $n_{j}=0$ are problematic. One approach is to fill them with some fictitious data before applying the weighted kernel estimate (3). Unfortunately, the construction of such data by some theoretically-supported procedure appears to be a challenging problem. We describe instead a fairly simple heuristic procedure.

Consider a given cell $R_{j}$ with $n_{j}=0$ but $\hat{n}_{j}>0$ and denote by $\bar{n}_{j}=\left\lceil n_{j}\right\rceil$ the smallest integer value larger than or equal to $\hat{n}_{j}$. The aim is to choose $\bar{n}_{j}$ fictitious points in $R_{j}$; recall that the points will be suitably weighted so that the overall weights of the cell will be $\hat{n}_{j}$.

An instinctive idea is to consider a component-wise average of the coordinates of some neighbouring observations falling in adjacent cells, but this may easily produce points outside the cell $R_{j}$. To avoid this problem, we consider instead an average of the coordinates of the corners of $R_{j}$, giving more weight to the corners closer to nearby observations. The specific procedure is as follows:

- the set of Euclidean distances of each observations from the centre of $R_{j}$ are computed and sorted in increasing order;

- construct a fictitious observation from the following two steps:

1. compute the Euclidean distances between the first element of the available observations (in the sorted list just constructed) and the $2^{d}$ corner points of $R_{j}$, and assign to each corner point a weight inversely proportional to the square root of its distance;

2. the above step is repeat $n_{a}$ times (in our work $n_{a}=3$ has been used), each time discarding the already employed observation from the sorted list, adding up the weights of the corner points; finally, retain the weighted average of the corner points as a new constructed observation;

- step 1 and 2 are repeated for each of the $\bar{n}_{j}$ points to be constructed.

\section{B. Output of the simulation study}

The definition of the distributions considered in the simulation study and the description of their parameters have been provided in Section 5. It remains to describe the non-random grid of points on which the estimation error has been evaluated. Consider the hypercube $(-q, q)^{d}$ where $q=6$ when $f(x)$ is a mixture with $0<\pi<1$ and $q=3$ is $\pi=1$. On the interval $[-q, q]$, select $N_{0}=\left\lceil N^{1 / d}\right\rceil$ equally spaced points, where $\lceil x\rceil$ is the smallest integer larger than or equal to $x$ and usually $N=2000$ was used. The Cartesian product of these coordinates for $d$ coordinated axes produces a grid of $N_{\mathrm{pts}}=N_{0}^{d}$ evaluation points.

The following pages provided a summary for each simulation run followed by an overall summary. 
Case No. 1

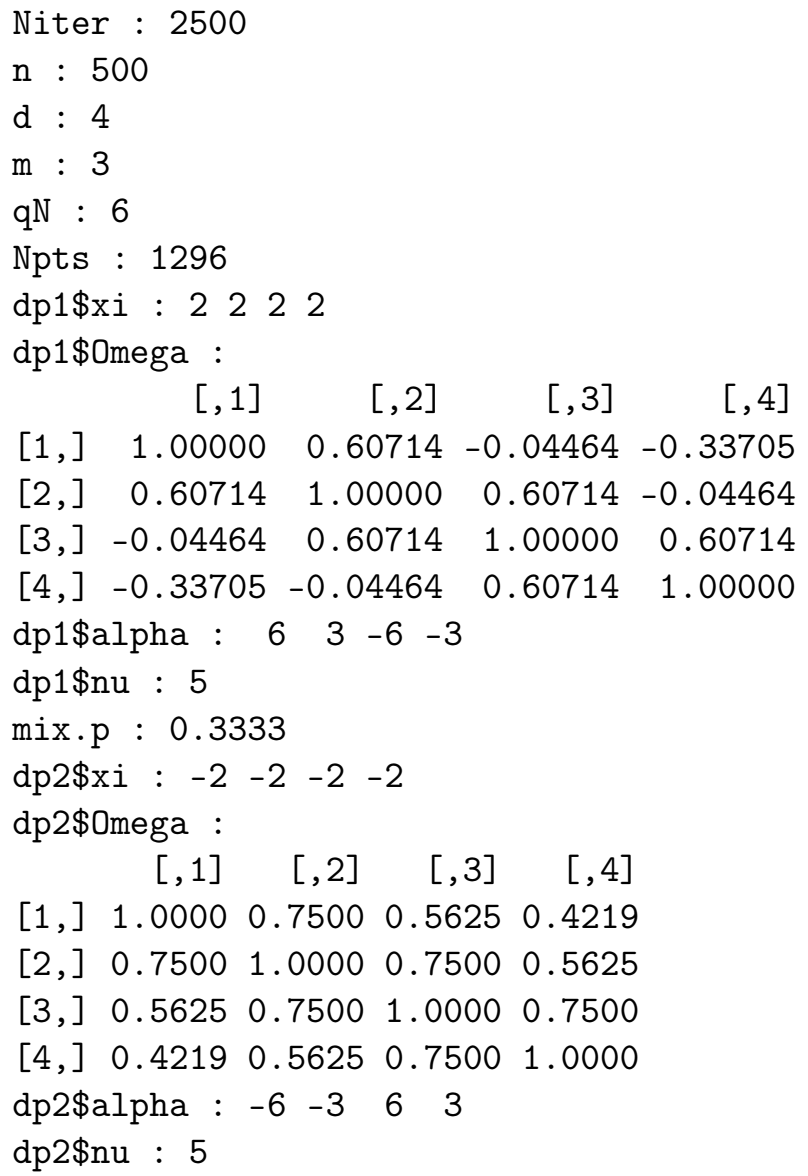

Table 2: Error quantiles for a fixed grid of points

\begin{tabular}{lllllll}
\hline & $25 \%$ & $50 \%$ & $75 \%$ & $90 \%$ & $95 \%$ & $99 \%$ \\
\hline e1 & $2.9 \mathrm{E}-05$ & $9.79 \mathrm{E}-05$ & 0.000348 & 0.00115 & 0.0028 & 0.0344 \\
e2 & $2.9 \mathrm{E}-05$ & $9.79 \mathrm{E}-05$ & 0.000348 & 0.00115 & 0.0028 & 0.034 \\
e3 & $2.89 \mathrm{E}-05$ & $9.79 \mathrm{E}-05$ & 0.000348 & 0.00115 & 0.0028 & 0.0341 \\
e4 & $2.89 \mathrm{E}-05$ & $9.79 \mathrm{E}-05$ & 0.00035 & 0.00118 & 0.00299 & 0.0371 \\
\hline
\end{tabular}

Table 3: Error quantiles evaluating at the observed sample points

\begin{tabular}{lllllll}
\hline & $25 \%$ & $50 \%$ & $75 \%$ & $90 \%$ & $95 \%$ & $99 \%$ \\
\hline $\mathrm{e} 1$ & 0.0274 & 0.06 & 0.114 & 0.183 & 0.232 & 0.636 \\
$\mathrm{e} 2$ & 0.0272 & 0.0596 & 0.114 & 0.182 & 0.232 & 0.634 \\
$\mathrm{e} 3$ & 0.0273 & 0.0597 & 0.114 & 0.182 & 0.232 & 0.634 \\
$\mathrm{e} 4$ & 0.0272 & 0.0596 & 0.114 & 0.182 & 0.232 & 0.63 \\
\hline
\end{tabular}


Case No. 2

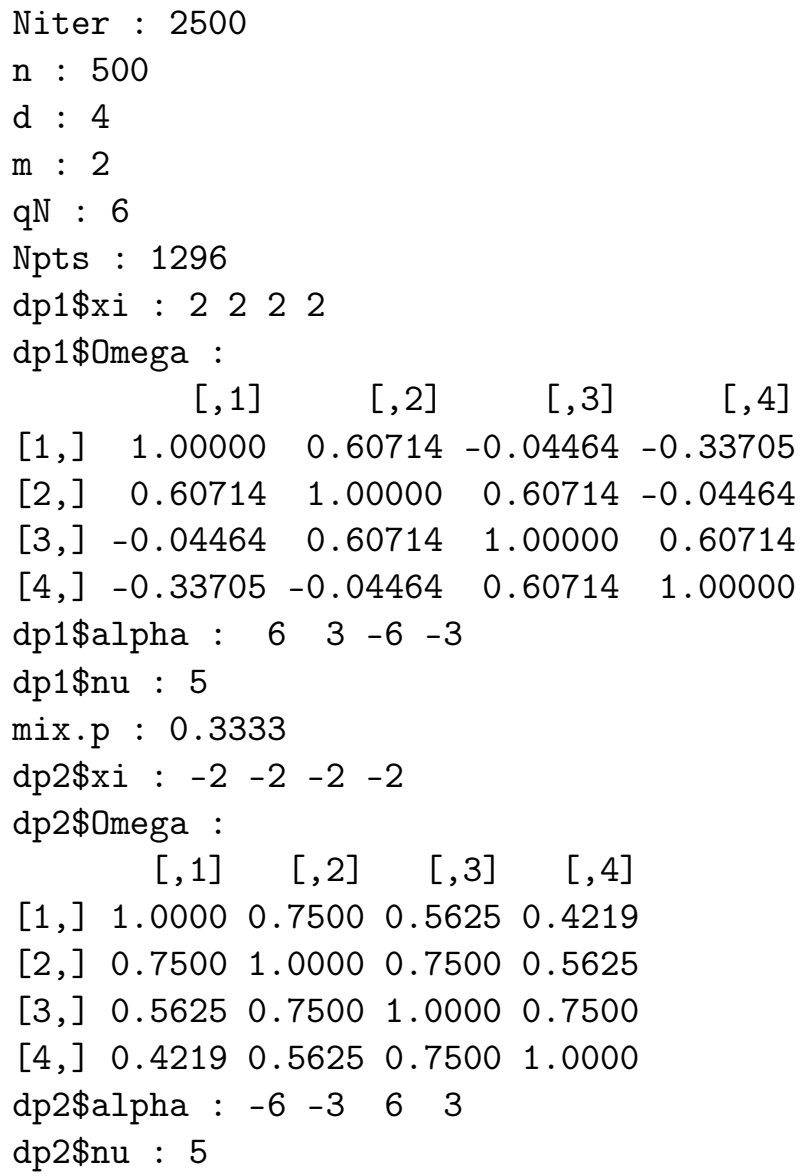

Table 4: Error quantiles for a fixed grid of points

\begin{tabular}{lllllll}
\hline & $25 \%$ & $50 \%$ & $75 \%$ & $90 \%$ & $95 \%$ & $99 \%$ \\
\hline e1 & $2.89 \mathrm{E}-05$ & $9.79 \mathrm{E}-05$ & 0.000348 & 0.00115 & 0.00279 & 0.0346 \\
e2 & $2.9 \mathrm{E}-05$ & $9.79 \mathrm{E}-05$ & 0.000348 & 0.00115 & 0.00272 & 0.0303 \\
e3 & $2.9 \mathrm{E}-05$ & $9.79 \mathrm{E}-05$ & 0.000348 & 0.00115 & 0.00273 & 0.0306 \\
e4 & $2.9 \mathrm{E}-05$ & $9.98 \mathrm{E}-05$ & 0.000371 & 0.00167 & 0.00581 & 0.0577 \\
\hline
\end{tabular}

Table 5: Error quantiles evaluating at the observed sample points

\begin{tabular}{lllllll}
\hline & $25 \%$ & $50 \%$ & $75 \%$ & $90 \%$ & $95 \%$ & $99 \%$ \\
\hline $\mathrm{e} 1$ & 0.0274 & 0.06 & 0.114 & 0.182 & 0.232 & 0.622 \\
$\mathrm{e} 2$ & 0.0255 & 0.0563 & 0.108 & 0.175 & 0.22 & 0.497 \\
$\mathrm{e} 3$ & 0.0261 & 0.0568 & 0.108 & 0.175 & 0.22 & 0.497 \\
$\mathrm{e} 4$ & 0.0252 & 0.0561 & 0.109 & 0.176 & 0.221 & 0.471 \\
\hline
\end{tabular}


Case No. 3

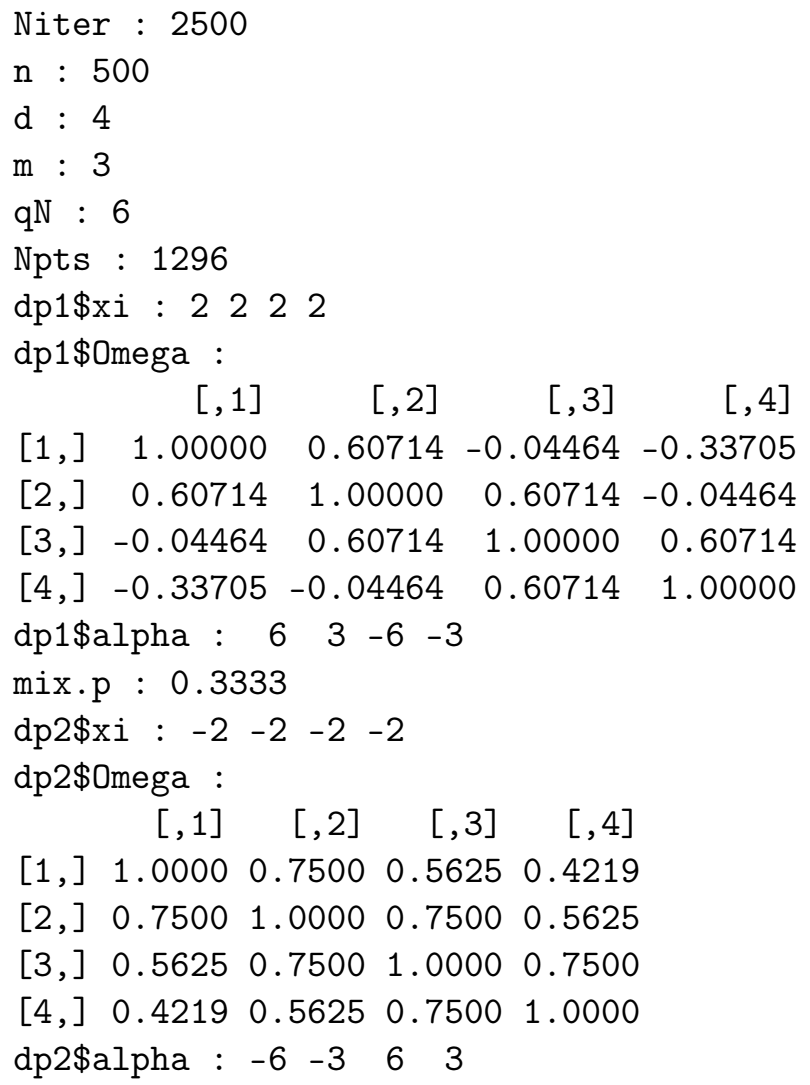

Table 6: Error quantiles for a fixed grid of points

\begin{tabular}{lllllll}
\hline & $25 \%$ & $50 \%$ & $75 \%$ & $90 \%$ & $95 \%$ & $99 \%$ \\
\hline e1 & $5.13 \mathrm{E}-22$ & $3.77 \mathrm{E}-12$ & $6.97 \mathrm{E}-05$ & $3.57 \mathrm{E}+03$ & $1.81 \mathrm{E}+13$ & $2.11 \mathrm{E}+36$ \\
$\mathrm{e} 2$ & $5.12 \mathrm{E}-22$ & $3.76 \mathrm{E}-12$ & $6.94 \mathrm{E}-05$ & $3.58 \mathrm{E}+03$ & $1.8 \mathrm{E}+13$ & $2.15 \mathrm{E}+36$ \\
$\mathrm{e} 3$ & $5.11 \mathrm{E}-22$ & $3.76 \mathrm{E}-12$ & $6.94 \mathrm{E}-05$ & $3.56 \mathrm{E}+03$ & $1.81 \mathrm{E}+13$ & $1.88 \mathrm{E}+36$ \\
e4 & $5.11 \mathrm{E}-20$ & $5.64 \mathrm{E}-11$ & 0.000709 & $1.25 \mathrm{E}+06$ & $1.19 \mathrm{E}+20$ & $1.2 \mathrm{E}+51$ \\
\hline
\end{tabular}

Table 7: Error quantiles evaluating at the observed sample points

\begin{tabular}{|c|c|c|c|c|c|c|}
\hline & $25 \%$ & $50 \%$ & $75 \%$ & $90 \%$ & $95 \%$ & $99 \%$ \\
\hline e1 & 0.0267 & 0.0569 & 0.0986 & 0.141 & 0.167 & 0.233 \\
\hline $\mathrm{e} 2$ & 0.0266 & 0.0568 & 0.0985 & 0.141 & 0.167 & 0.233 \\
\hline e3 & 0.0266 & 0.0568 & 0.0985 & 0.141 & 0.167 & 0.233 \\
\hline e4 & 0.0266 & 0.0569 & 0.0986 & 0.141 & 0.167 & 0.233 \\
\hline
\end{tabular}


Case No. 4

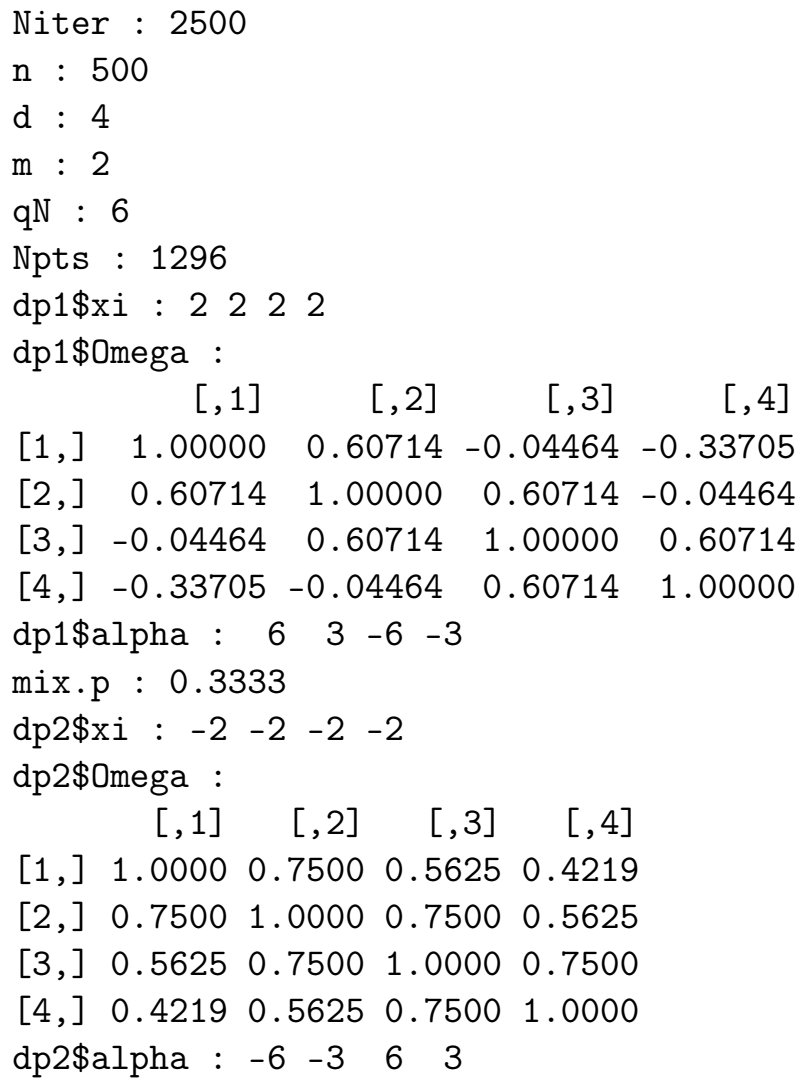

Table 8: Error quantiles for a fixed grid of points

\begin{tabular}{lllllll}
\hline & $25 \%$ & $50 \%$ & $75 \%$ & $90 \%$ & $95 \%$ & $99 \%$ \\
\hline kde & $5.14 \mathrm{E}-22$ & $3.79 \mathrm{E}-12$ & $7.13 \mathrm{E}-05$ & $4.21 \mathrm{E}+03$ & $3.22 \mathrm{E}+13$ & $1.29 \mathrm{E}+36$ \\
wkde & $5.01 \mathrm{E}-22$ & $3.68 \mathrm{E}-12$ & $6.75 \mathrm{E}-05$ & $4.02 \mathrm{E}+03$ & $3.03 \mathrm{E}+13$ & $1.25 \mathrm{E}+36$ \\
wkdeA & $5 \mathrm{E}-22$ & $3.7 \mathrm{E}-12$ & $6.84 \mathrm{E}-05$ & $4.06 \mathrm{E}+03$ & $2.92 \mathrm{E}+13$ & $1.19 \mathrm{E}+36$ \\
fill+wkde & $4.55 \mathrm{E}-16$ & $4.4 \mathrm{E}-08$ & 0.277 & $8.34 \mathrm{E}+12$ & $9.88 \mathrm{E}+27$ & $1.56 \mathrm{E}+61$ \\
\hline
\end{tabular}

Table 9: Error quantiles evaluating at the observed sample points

\begin{tabular}{lllllll}
\hline & $25 \%$ & $50 \%$ & $75 \%$ & $90 \%$ & $95 \%$ & $99 \%$ \\
\hline kde & 0.0266 & 0.057 & 0.0986 & 0.141 & 0.167 & 0.234 \\
wkde & 0.0264 & 0.0565 & 0.098 & 0.14 & 0.166 & 0.232 \\
wkdeA & 0.0263 & 0.0563 & 0.0976 & 0.14 & 0.165 & 0.23 \\
fill+wkde & 0.0265 & 0.0568 & 0.0985 & 0.141 & 0.166 & 0.231 \\
\hline
\end{tabular}




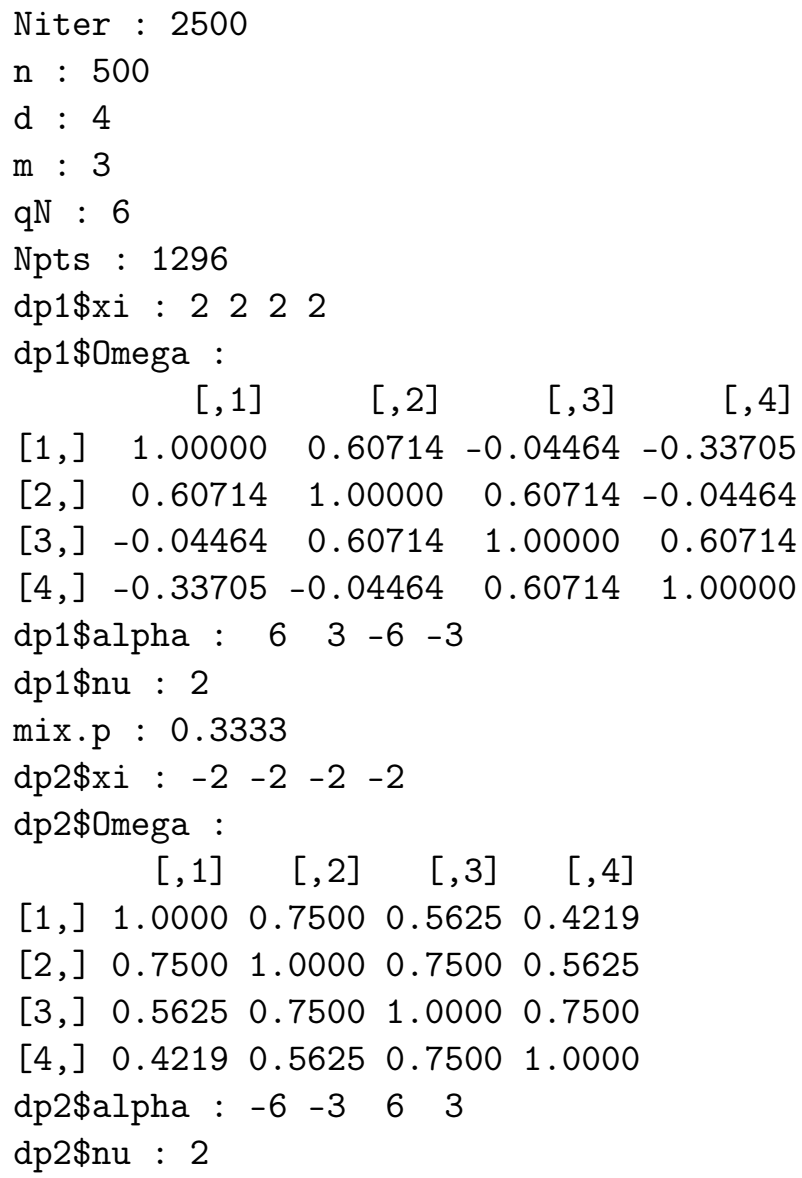

Table 10: Error quantiles for a fixed grid of points

\begin{tabular}{lllllll}
\hline & $25 \%$ & $50 \%$ & $75 \%$ & $90 \%$ & $95 \%$ & $99 \%$ \\
\hline kde & 0.000202 & 0.000462 & 0.00115 & 0.00321 & 0.00813 & 0.0485 \\
wkde & 0.000202 & 0.000462 & 0.00116 & 0.00322 & 0.00818 & 0.0486 \\
wkdeA & 0.000202 & 0.000462 & 0.00116 & 0.00322 & 0.00818 & 0.0486 \\
fill+wkde & 0.000202 & 0.000462 & 0.00115 & 0.00321 & 0.00816 & 0.0486 \\
\hline
\end{tabular}

Table 11: Error quantiles evaluating at the observed sample points

\begin{tabular}{lllllll}
\hline & $25 \%$ & $50 \%$ & $75 \%$ & $90 \%$ & $95 \%$ & $99 \%$ \\
\hline kde & 0.025 & 0.0586 & 0.136 & 0.246 & 0.343 & 2.63 \\
wkde & 0.025 & 0.0587 & 0.136 & 0.247 & 0.345 & 2.67 \\
wkdeA & 0.025 & 0.0587 & 0.136 & 0.246 & 0.345 & 2.66 \\
fill+wkde & 0.0249 & 0.0585 & 0.136 & 0.246 & 0.343 & 2.62 \\
\hline
\end{tabular}


Case No. 6

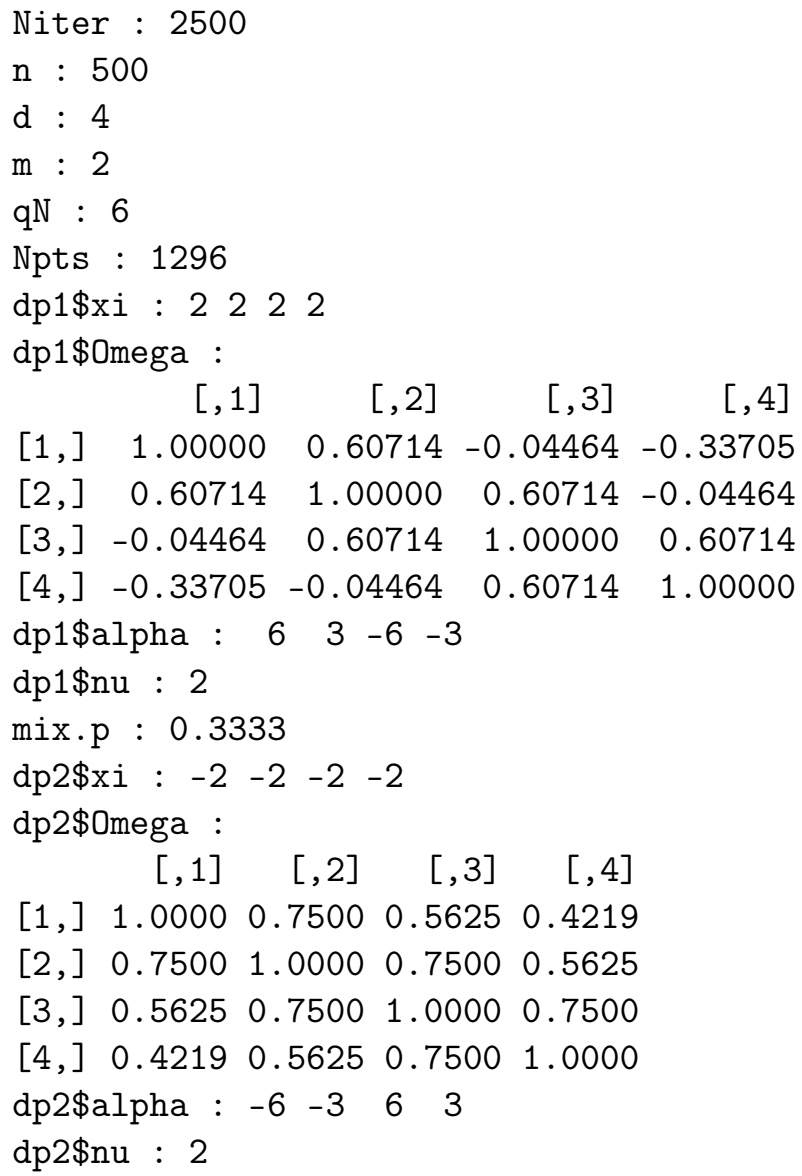

Table 12: Error quantiles for a fixed grid of points

\begin{tabular}{lllllll}
\hline & $25 \%$ & $50 \%$ & $75 \%$ & $90 \%$ & $95 \%$ & $99 \%$ \\
\hline kde & 0.000208 & 0.000481 & 0.00121 & 0.00331 & 0.00827 & 0.0487 \\
wkde & 0.00021 & 0.000486 & 0.00122 & 0.00319 & 0.00689 & 0.0398 \\
wkdeA & 0.00021 & 0.000486 & 0.00121 & 0.00319 & 0.00694 & 0.0396 \\
fill+wkde & 0.000205 & 0.000478 & 0.00121 & 0.00329 & 0.00775 & 0.0443 \\
\hline
\end{tabular}

Table 13: Error quantiles evaluating at the observed sample points

\begin{tabular}{lllllll}
\hline & $25 \%$ & $50 \%$ & $75 \%$ & $90 \%$ & $95 \%$ & $99 \%$ \\
\hline kde & 0.025 & 0.0587 & 0.135 & 0.245 & 0.341 & 2.6 \\
wkde & 0.0182 & 0.0494 & 0.125 & 0.234 & 0.323 & 2.95 \\
wkdeA & 0.0188 & 0.0496 & 0.125 & 0.234 & 0.323 & 2.95 \\
fill+wkde & 0.0181 & 0.0485 & 0.124 & 0.228 & 0.302 & 1.73 \\
\hline
\end{tabular}


Case No. 7

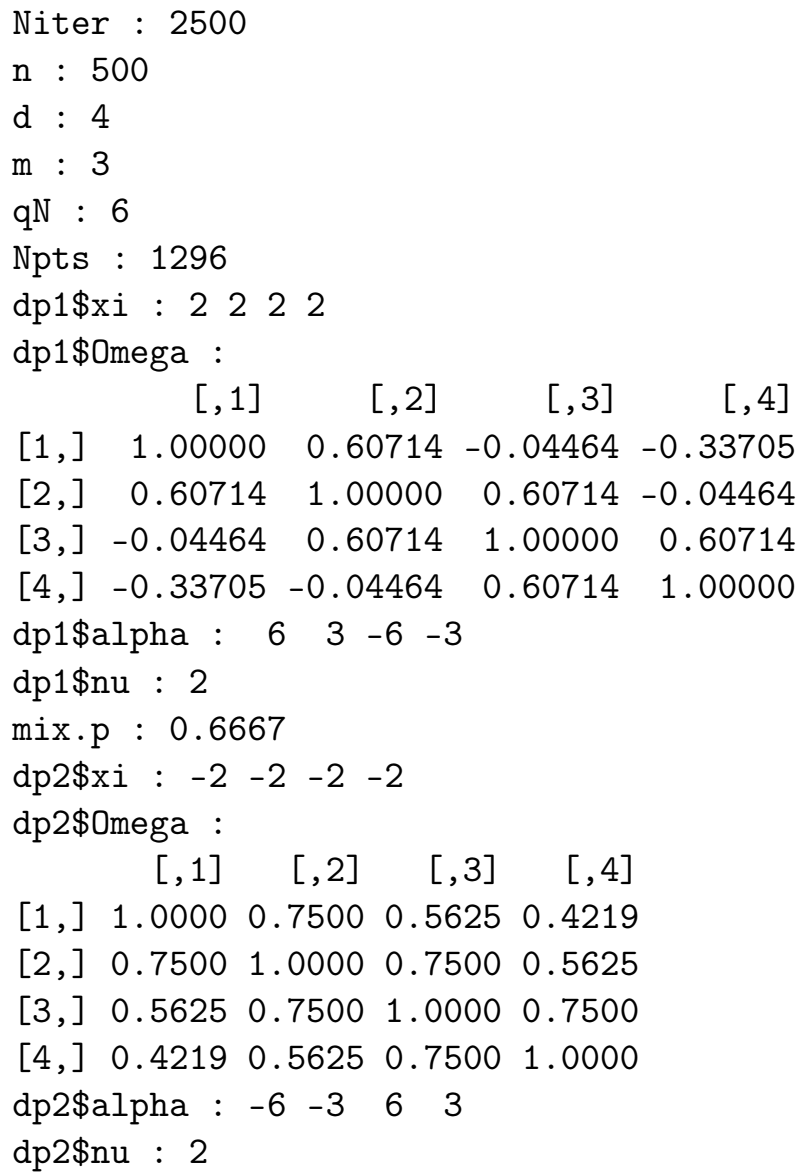

Table 14: Error quantiles for a fixed grid of points

\begin{tabular}{lllllll}
\hline & $25 \%$ & $50 \%$ & $75 \%$ & $90 \%$ & $95 \%$ & $99 \%$ \\
\hline kde & 0.000182 & 0.000429 & 0.00112 & 0.00323 & 0.00812 & 0.0477 \\
wkde & 0.000182 & 0.000429 & 0.00112 & 0.00324 & 0.00815 & 0.0479 \\
wkdeA & 0.000182 & 0.000429 & 0.00112 & 0.00324 & 0.00815 & 0.0479 \\
fill+wkde & 0.000182 & 0.000429 & 0.00112 & 0.00324 & 0.00814 & 0.0477 \\
\hline
\end{tabular}

Table 15: Error quantiles evaluating at the observed sample points

\begin{tabular}{lllllll}
\hline & $25 \%$ & $50 \%$ & $75 \%$ & $90 \%$ & $95 \%$ & $99 \%$ \\
\hline kde & 0.025 & 0.0614 & 0.141 & 0.254 & 0.355 & 2.65 \\
wkde & 0.0251 & 0.0616 & 0.142 & 0.254 & 0.357 & 2.69 \\
wkdeA & 0.0251 & 0.0616 & 0.142 & 0.254 & 0.357 & 2.69 \\
fill+wkde & 0.025 & 0.0614 & 0.141 & 0.254 & 0.355 & 2.65 \\
\hline
\end{tabular}


Case No. 8

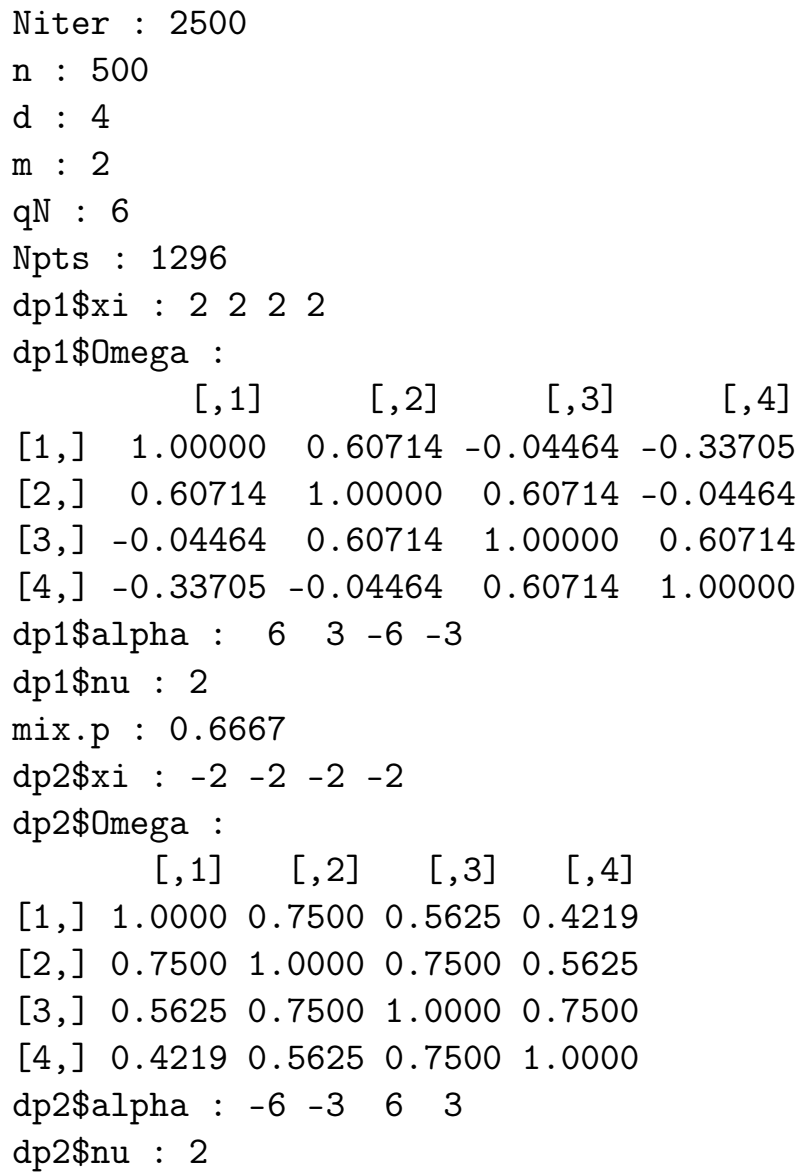

Table 16: Error quantiles for a fixed grid of points

\begin{tabular}{lllllll}
\hline & $25 \%$ & $50 \%$ & $75 \%$ & $90 \%$ & $95 \%$ & $99 \%$ \\
\hline kde & 0.000182 & 0.000433 & 0.00113 & 0.00321 & 0.00806 & 0.0478 \\
wkde & 0.000184 & 0.000439 & 0.00114 & 0.00304 & 0.00702 & 0.0392 \\
wkdeA & 0.000184 & 0.000439 & 0.00114 & 0.00305 & 0.00707 & 0.039 \\
fill+wkde & 0.000179 & 0.000426 & 0.00113 & 0.00324 & 0.00769 & 0.0417 \\
\hline
\end{tabular}

Table 17: Error quantiles evaluating at the observed sample points

\begin{tabular}{lllllll}
\hline & $25 \%$ & $50 \%$ & $75 \%$ & $90 \%$ & $95 \%$ & $99 \%$ \\
\hline kde & 0.0251 & 0.0616 & 0.141 & 0.254 & 0.355 & 2.73 \\
wkde & 0.0211 & 0.0552 & 0.131 & 0.243 & 0.337 & 3.06 \\
wkdeA & 0.0218 & 0.0554 & 0.131 & 0.243 & 0.338 & 3.07 \\
fill+wkde & 0.0184 & 0.0514 & 0.13 & 0.235 & 0.314 & 1.71 \\
\hline
\end{tabular}


Case No. 9

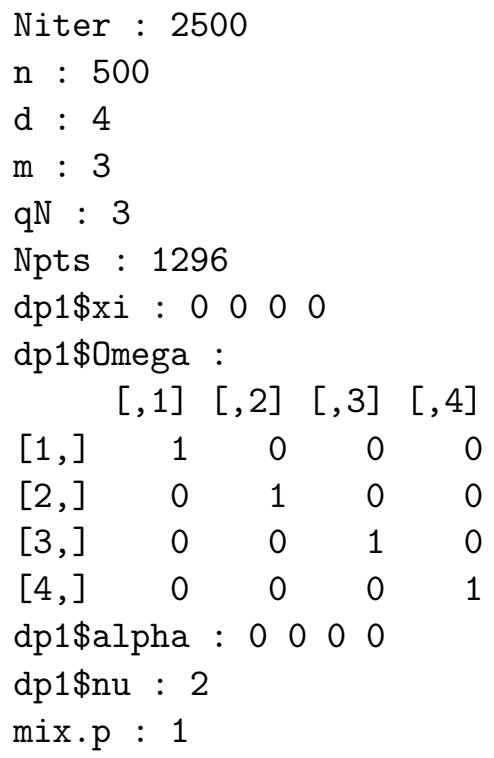

Table 18: Error quantiles for a fixed grid of points

\begin{tabular}{lllllll}
\hline & $25 \%$ & $50 \%$ & $75 \%$ & $90 \%$ & $95 \%$ & $99 \%$ \\
\hline kde & 0.00304 & 0.00495 & 0.00858 & 0.0182 & 0.0268 & 0.0472 \\
wkde & 0.00304 & 0.00496 & 0.00861 & 0.0183 & 0.027 & 0.0475 \\
wkdeA & 0.00304 & 0.00496 & 0.00861 & 0.0183 & 0.027 & 0.0475 \\
fill+wkde & 0.00304 & 0.00495 & 0.00857 & 0.0182 & 0.0268 & 0.0471 \\
\hline
\end{tabular}

Table 19: Error quantiles evaluating at the observed sample points

\begin{tabular}{lllllll}
\hline & $25 \%$ & $50 \%$ & $75 \%$ & $90 \%$ & $95 \%$ & $99 \%$ \\
\hline kde & 0.0158 & 0.0326 & 0.0707 & 0.185 & 0.506 & 5.55 \\
wkde & 0.0158 & 0.0328 & 0.0712 & 0.186 & 0.509 & 5.57 \\
wkdeA & 0.0158 & 0.0328 & 0.0713 & 0.187 & 0.509 & 5.57 \\
fill+wkde & 0.0157 & 0.0325 & 0.0706 & 0.185 & 0.504 & 5.52 \\
\hline
\end{tabular}




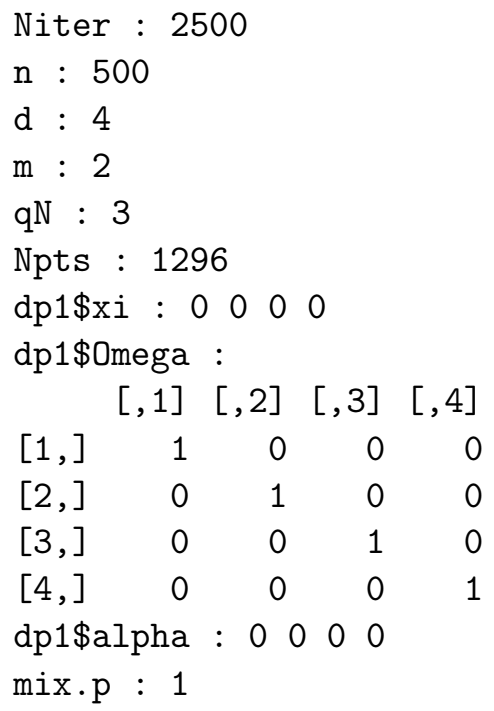

Table 20: Error quantiles for a fixed grid of points

\begin{tabular}{lllllll}
\hline & $25 \%$ & $50 \%$ & $75 \%$ & $90 \%$ & $95 \%$ & $99 \%$ \\
\hline kde & 0.00071 & 0.00213 & 0.00624 & 0.0197 & 0.0349 & 0.0938 \\
wkde & 0.000712 & 0.00215 & 0.00614 & 0.018 & 0.0306 & 0.0815 \\
wkdeA & 0.000712 & 0.00213 & 0.00615 & 0.018 & 0.0308 & 0.0812 \\
fill+wkde & 0.000976 & 0.00403 & 0.0142 & 0.0364 & 0.0585 & 0.125 \\
\hline
\end{tabular}

Table 21: Error quantiles evaluating at the observed sample points

\begin{tabular}{lllllll}
\hline & $25 \%$ & $50 \%$ & $75 \%$ & $90 \%$ & $95 \%$ & $99 \%$ \\
\hline kde & 0.0152 & 0.0334 & 0.0643 & 0.113 & 0.164 & 0.388 \\
wkde & 0.0149 & 0.0329 & 0.0617 & 0.103 & 0.146 & 0.335 \\
wkdeA & 0.0151 & 0.0327 & 0.0601 & 0.1 & 0.143 & 0.333 \\
fill+wkde & 0.0108 & 0.0228 & 0.0406 & 0.0715 & 0.106 & 0.239 \\
\hline
\end{tabular}




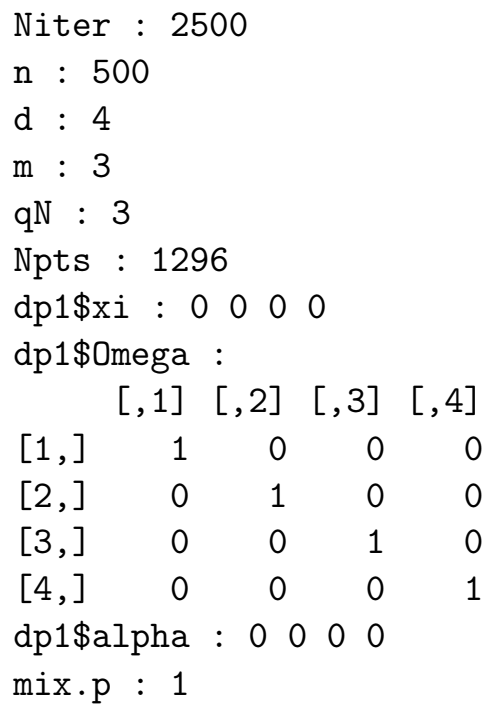

Table 22: Error quantiles for a fixed grid of points

\begin{tabular}{lllllll}
\hline & $25 \%$ & $50 \%$ & $75 \%$ & $90 \%$ & $95 \%$ & $99 \%$ \\
\hline kde & 0.00071 & 0.00214 & 0.00625 & 0.0198 & 0.0349 & 0.0942 \\
wkde & 0.000712 & 0.00218 & 0.00622 & 0.0195 & 0.0342 & 0.0937 \\
wkdeA & 0.000711 & 0.00215 & 0.00623 & 0.0196 & 0.0344 & 0.0931 \\
fill+wkde & 0.000646 & 0.00257 & 0.00921 & 0.026 & 0.0435 & 0.105 \\
\hline
\end{tabular}

Table 23: Error quantiles evaluating at the observed sample points

\begin{tabular}{lllllll}
\hline & $25 \%$ & $50 \%$ & $75 \%$ & $90 \%$ & $95 \%$ & $99 \%$ \\
\hline kde & 0.0152 & 0.0334 & 0.0643 & 0.113 & 0.164 & 0.389 \\
wkde & 0.0153 & 0.0338 & 0.0651 & 0.114 & 0.164 & 0.387 \\
wkdeA & 0.0153 & 0.0337 & 0.0648 & 0.113 & 0.162 & 0.382 \\
fill+wkde & 0.0126 & 0.0275 & 0.0532 & 0.0979 & 0.143 & 0.336 \\
\hline
\end{tabular}


Case No. 12

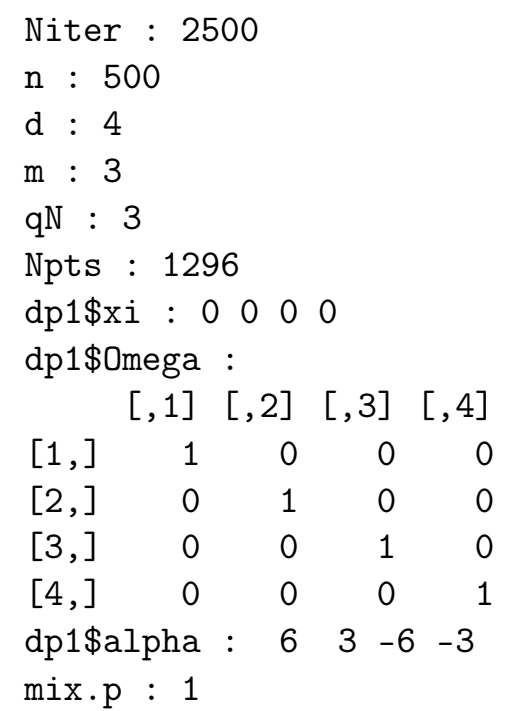

Table 24: Error quantiles for a fixed grid of points

\begin{tabular}{lllllll}
\hline & $25 \%$ & $50 \%$ & $75 \%$ & $90 \%$ & $95 \%$ & $99 \%$ \\
\hline kde & 0.00143 & 0.0127 & $3.02 \mathrm{E}+12$ & $8.48 \mathrm{E}+53$ & $3.35 \mathrm{E}+90$ & Inf \\
wkde & 0.00144 & 0.0127 & $2.94 \mathrm{E}+12$ & $7.96 \mathrm{E}+53$ & $2.92 \mathrm{E}+90$ & Inf \\
wkdeA & 0.00143 & 0.0127 & $3.05 \mathrm{E}+12$ & $8.25 \mathrm{E}+53$ & $3.1 \mathrm{E}+90$ & Inf \\
fill+wkde & 0.00165 & 0.016 & $1.55 \mathrm{E}+14$ & $1.08 \mathrm{E}+60$ & $4.45 \mathrm{E}+100$ & Inf \\
\hline
\end{tabular}

Table 25: Error quantiles evaluating at the observed sample points

\begin{tabular}{lllllll}
\hline & $25 \%$ & $50 \%$ & $75 \%$ & $90 \%$ & $95 \%$ & $99 \%$ \\
\hline kde & 0.0198 & 0.0428 & 0.0796 & 0.14 & 0.206 & 0.49 \\
wkde & 0.0197 & 0.0428 & 0.0805 & 0.141 & 0.207 & 0.488 \\
wkdeA & 0.0198 & 0.043 & 0.0801 & 0.141 & 0.205 & 0.485 \\
fill+wkde & 0.0177 & 0.0385 & 0.0718 & 0.126 & 0.187 & 0.439 \\
\hline
\end{tabular}




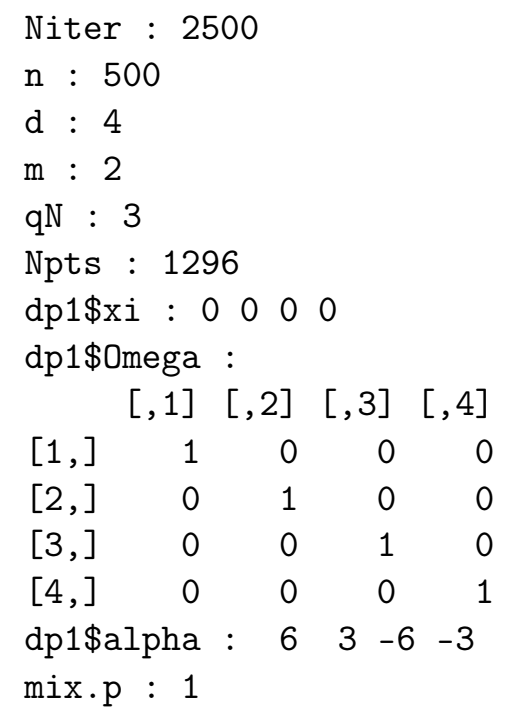

Table 26: Error quantiles for a fixed grid of points

\begin{tabular}{lllllll}
\hline & $25 \%$ & $50 \%$ & $75 \%$ & $90 \%$ & $95 \%$ & $99 \%$ \\
\hline kde & 0.00143 & 0.0127 & $2.91 \mathrm{E}+12$ & $8.23 \mathrm{E}+53$ & $3.23 \mathrm{E}+90$ & Inf \\
wkde & 0.00141 & 0.0121 & $1.94 \mathrm{E}+12$ & $5.96 \mathrm{E}+53$ & $2.23 \mathrm{E}+90$ & Inf \\
wkdeA & 0.00141 & 0.0121 & $2.17 \mathrm{E}+12$ & $6.34 \mathrm{E}+53$ & $2.42 \mathrm{E}+90$ & Inf \\
fill+wkde & 0.00219 & 0.023 & $2.33 \mathrm{E}+16$ & $1.55 \mathrm{E}+63$ & $3.62 \mathrm{E}+105$ & Inf \\
\hline
\end{tabular}

Table 27: Error quantiles evaluating at the observed sample points

\begin{tabular}{lllllll}
\hline & $25 \%$ & $50 \%$ & $75 \%$ & $90 \%$ & $95 \%$ & $99 \%$ \\
\hline kde & 0.0197 & 0.0426 & 0.0794 & 0.14 & 0.205 & 0.487 \\
wkde & 0.0189 & 0.0414 & 0.0778 & 0.135 & 0.195 & 0.458 \\
wkdeA & 0.019 & 0.0413 & 0.0761 & 0.131 & 0.19 & 0.448 \\
fill+wkde & 0.0157 & 0.0341 & 0.0621 & 0.0997 & 0.144 & 0.336 \\
\hline
\end{tabular}


Case No. 14

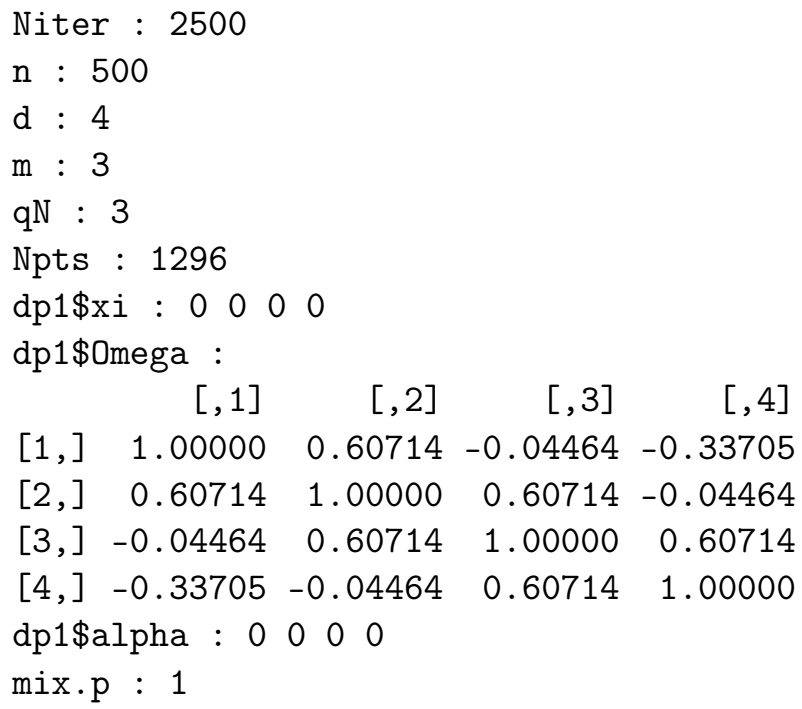

Table 28: Error quantiles for a fixed grid of points

\begin{tabular}{lllllll}
\hline & $25 \%$ & $50 \%$ & $75 \%$ & $90 \%$ & $95 \%$ & $99 \%$ \\
\hline kde & $2.01 \mathrm{E}-05$ & 0.00195 & 0.033 & 0.283 & 1.31 & 122 \\
wkde & $1.98 \mathrm{E}-05$ & 0.00193 & 0.0325 & 0.269 & 1.2 & 104 \\
wkdeA & $1.99 \mathrm{E}-05$ & 0.00194 & 0.0327 & 0.273 & 1.23 & 107 \\
fill+wkde & $8.63 \mathrm{E}-05$ & 0.00794 & 0.429 & $5.43 \mathrm{E}+03$ & $3.73 \mathrm{E}+06$ & $1.02 \mathrm{E}+12$ \\
\hline
\end{tabular}

Table 29: Error quantiles evaluating at the observed sample points

\begin{tabular}{lllllll}
\hline & $25 \%$ & $50 \%$ & $75 \%$ & $90 \%$ & $95 \%$ & $99 \%$ \\
\hline kde & 0.0265 & 0.0561 & 0.0977 & 0.158 & 0.228 & 0.5 \\
wkde & 0.0265 & 0.0562 & 0.0977 & 0.157 & 0.227 & 0.498 \\
wkdeA & 0.0265 & 0.0562 & 0.0978 & 0.157 & 0.227 & 0.498 \\
fill+wkde & 0.0266 & 0.0562 & 0.0973 & 0.155 & 0.223 & 0.49 \\
\hline
\end{tabular}


Case No. 15

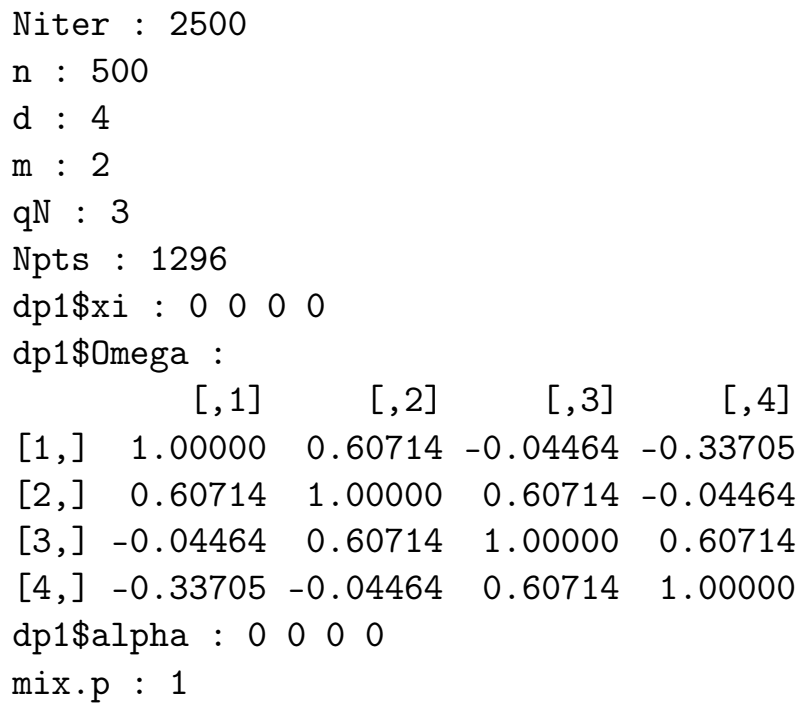

Table 30: Error quantiles for a fixed grid of points

\begin{tabular}{lllllll}
\hline & $25 \%$ & $50 \%$ & $75 \%$ & $90 \%$ & $95 \%$ & $99 \%$ \\
\hline kde & $2 \mathrm{E}-05$ & 0.00194 & 0.0328 & 0.274 & 1.2 & 203 \\
wkde & $1.55 \mathrm{E}-05$ & 0.00159 & 0.0267 & 0.202 & 0.742 & 28.1 \\
wkdeA & $1.66 \mathrm{E}-05$ & 0.00169 & 0.0284 & 0.218 & 0.831 & 38.7 \\
fill + wkde & 0.00882 & 0.391 & $9.27 \mathrm{E}+03$ & $3.55 \mathrm{E}+10$ & $1.78 \mathrm{E}+15$ & $6.86 \mathrm{E}+25$ \\
\hline
\end{tabular}

Table 31: Error quantiles evaluating at the observed sample points

\begin{tabular}{lllllll}
\hline & $25 \%$ & $50 \%$ & $75 \%$ & $90 \%$ & $95 \%$ & $99 \%$ \\
\hline kde & 0.0265 & 0.0561 & 0.0976 & 0.158 & 0.229 & 0.503 \\
wkde & 0.0249 & 0.053 & 0.0928 & 0.149 & 0.215 & 0.472 \\
wkdeA & 0.0249 & 0.0527 & 0.092 & 0.148 & 0.211 & 0.458 \\
fill+wkde & 0.0257 & 0.0543 & 0.0926 & 0.138 & 0.191 & 0.423 \\
\hline
\end{tabular}




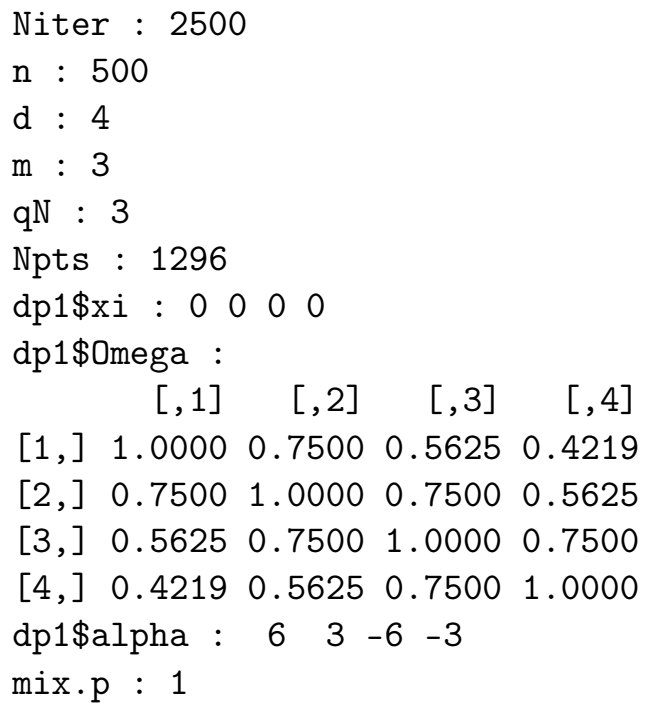

Table 32: Error quantiles for a fixed grid of points

\begin{tabular}{lllllll}
\hline & $25 \%$ & $50 \%$ & $75 \%$ & $90 \%$ & $95 \%$ & $99 \%$ \\
\hline kde & $3.11 \mathrm{E}-07$ & 0.000968 & $4.54 \mathrm{E}+07$ & $3.54 \mathrm{E}+48$ & $3.09 \mathrm{E}+82$ & Inf \\
wkde & $3.1 \mathrm{E}-07$ & 0.000953 & $4.43 \mathrm{E}+07$ & $3.39 \mathrm{E}+48$ & $3.02 \mathrm{E}+82$ & Inf \\
wkdeA & $3.11 \mathrm{E}-07$ & 0.00096 & $4.48 \mathrm{E}+07$ & $3.48 \mathrm{E}+48$ & $3.03 \mathrm{E}+82$ & Inf \\
fill+wkde & $7.65 \mathrm{E}-07$ & 0.00272 & $7.55 \mathrm{E}+08$ & $1.14 \mathrm{E}+53$ & $1.31 \mathrm{E}+88$ & Inf \\
\hline
\end{tabular}

Table 33: Error quantiles evaluating at the observed sample points

\begin{tabular}{lllllll}
\hline & $25 \%$ & $50 \%$ & $75 \%$ & $90 \%$ & $95 \%$ & $99 \%$ \\
\hline kde & 0.0312 & 0.0665 & 0.117 & 0.184 & 0.257 & 0.58 \\
wkde & 0.0306 & 0.0653 & 0.115 & 0.182 & 0.256 & 0.58 \\
wkdeA & 0.0306 & 0.0654 & 0.116 & 0.182 & 0.255 & 0.579 \\
fill+wkde & 0.0306 & 0.0653 & 0.115 & 0.179 & 0.25 & 0.568 \\
\hline
\end{tabular}




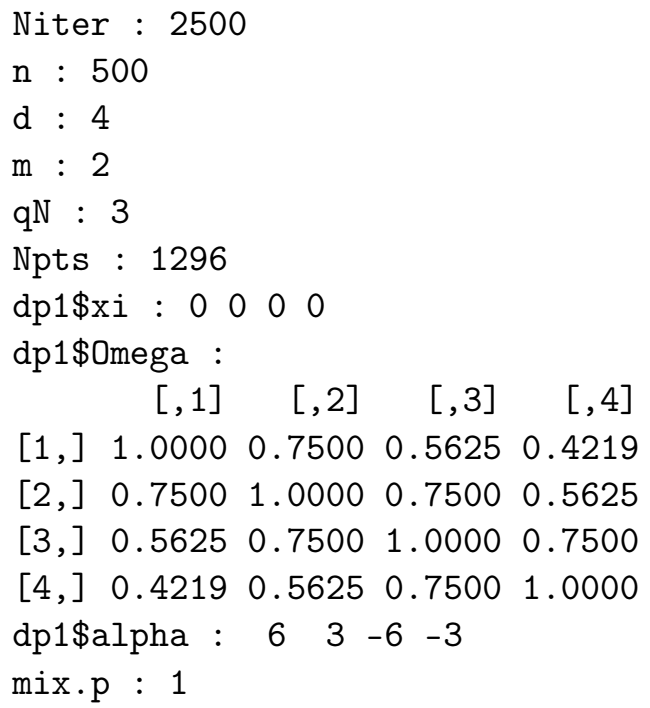

Table 34: Error quantiles for a fixed grid of points

\begin{tabular}{lllllll}
\hline & $25 \%$ & $50 \%$ & $75 \%$ & $90 \%$ & $95 \%$ & $99 \%$ \\
\hline kde & $3.1 \mathrm{E}-07$ & 0.00096 & $4.36 \mathrm{E}+07$ & $3.15 \mathrm{E}+48$ & $1.37 \mathrm{E}+82$ & Inf \\
wkde & $2.85 \mathrm{E}-07$ & 0.000778 & $2.73 \mathrm{E}+07$ & $1.89 \mathrm{E}+48$ & $8.26 \mathrm{E}+81$ & Inf \\
wkdeA & $2.9 \mathrm{E}-07$ & 0.000807 & $3.02 \mathrm{E}+07$ & $2.14 \mathrm{E}+48$ & $8.73 \mathrm{E}+81$ & Inf \\
fill+wkde & 0.000213 & 0.0343 & $9.94 \mathrm{E}+13$ & $7.86 \mathrm{E}+59$ & $3.93 \mathrm{E}+99$ & Inf \\
\hline
\end{tabular}

Table 35: Error quantiles evaluating at the observed sample points

\begin{tabular}{lllllll}
\hline & $25 \%$ & $50 \%$ & $75 \%$ & $90 \%$ & $95 \%$ & $99 \%$ \\
\hline kde & 0.0312 & 0.0665 & 0.117 & 0.184 & 0.257 & 0.574 \\
wkde & 0.0274 & 0.0592 & 0.106 & 0.168 & 0.235 & 0.531 \\
wkdeA & 0.0273 & 0.0588 & 0.105 & 0.165 & 0.229 & 0.518 \\
fill+wkde & 0.0274 & 0.059 & 0.105 & 0.16 & 0.211 & 0.475 \\
\hline
\end{tabular}




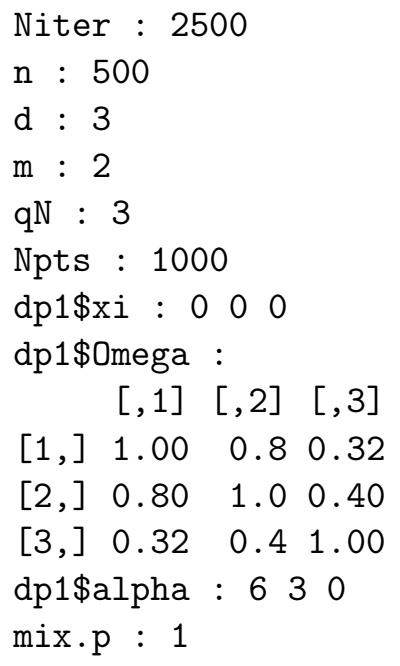

Table 36: Error quantiles for a fixed grid of points

\begin{tabular}{lllllll}
\hline & $25 \%$ & $50 \%$ & $75 \%$ & $90 \%$ & $95 \%$ & $99 \%$ \\
\hline kde & $5.5 \mathrm{E}-06$ & 0.00257 & 0.0482 & $5.2 \mathrm{E}+03$ & $3.79 \mathrm{E}+08$ & $4.83 \mathrm{E}+14$ \\
wkde & $5.23 \mathrm{E}-06$ & 0.00243 & 0.0436 & $5.26 \mathrm{E}+03$ & $2.59 \mathrm{E}+08$ & $3.08 \mathrm{E}+14$ \\
wkdeA & $5.32 \mathrm{E}-06$ & 0.00244 & 0.0438 & $4.1 \mathrm{E}+03$ & $2.66 \mathrm{E}+08$ & $3.18 \mathrm{E}+14$ \\
fill+wkde & $2.73 \mathrm{E}-05$ & 0.0091 & 0.149 & $6.56 \mathrm{E}+07$ & $3.94 \mathrm{E}+13$ & $1.33 \mathrm{E}+22$ \\
\hline
\end{tabular}

Table 37: Error quantiles evaluating at the observed sample points

\begin{tabular}{lllllll}
\hline & $25 \%$ & $50 \%$ & $75 \%$ & $90 \%$ & $95 \%$ & $99 \%$ \\
\hline kde & 0.0256 & 0.0541 & 0.0941 & 0.144 & 0.189 & 0.357 \\
wkde & 0.0238 & 0.0505 & 0.0882 & 0.135 & 0.177 & 0.335 \\
wkdeA & 0.0234 & 0.0497 & 0.0866 & 0.133 & 0.173 & 0.327 \\
fill+wkde & 0.0239 & 0.0504 & 0.0872 & 0.132 & 0.17 & 0.324 \\
\hline
\end{tabular}




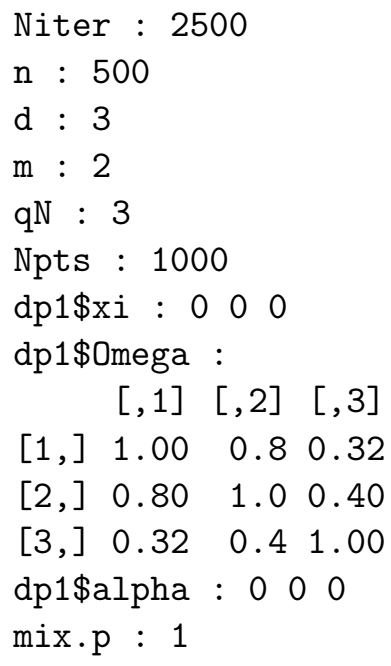

Table 38: Error quantiles for a fixed grid of points

\begin{tabular}{lllllll}
\hline & $25 \%$ & $50 \%$ & $75 \%$ & $90 \%$ & $95 \%$ & $99 \%$ \\
\hline kde & $8.01 \mathrm{E}-05$ & 0.00341 & 0.0216 & 0.0572 & 0.0879 & 0.187 \\
wkde & $7.23 \mathrm{E}-05$ & 0.00298 & 0.0193 & 0.0501 & 0.0767 & 0.156 \\
wkdeA & $7.29 \mathrm{E}-05$ & 0.00303 & 0.0195 & 0.0501 & 0.0762 & 0.153 \\
fill+wkde & 0.00148 & 0.0128 & 0.0468 & 0.146 & 0.475 & 33.2 \\
\hline
\end{tabular}

Table 39: Error quantiles evaluating at the observed sample points

\begin{tabular}{lllllll}
\hline & $25 \%$ & $50 \%$ & $75 \%$ & $90 \%$ & $95 \%$ & $99 \%$ \\
\hline kde & 0.0198 & 0.0415 & 0.0713 & 0.108 & 0.141 & 0.258 \\
wkde & 0.0186 & 0.039 & 0.0669 & 0.101 & 0.13 & 0.237 \\
wkdeA & 0.0184 & 0.0386 & 0.0662 & 0.0992 & 0.128 & 0.232 \\
fill+wkde & 0.019 & 0.0397 & 0.0674 & 0.0991 & 0.126 & 0.232 \\
\hline
\end{tabular}


Case No. 20

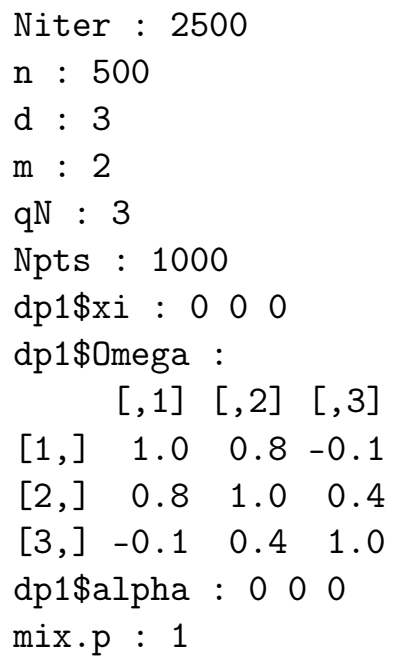

Table 40: Error quantiles for a fixed grid of points

\begin{tabular}{lllllll}
\hline & $25 \%$ & $50 \%$ & $75 \%$ & $90 \%$ & $95 \%$ & $99 \%$ \\
\hline kde & $3.96 \mathrm{E}-05$ & 0.00577 & 0.0462 & 0.143 & 0.248 & 1.12 \\
wkde & $3.3 \mathrm{E}-05$ & 0.00534 & 0.0436 & 0.131 & 0.219 & 0.777 \\
wkdeA & $3.47 \mathrm{E}-05$ & 0.00544 & 0.0441 & 0.133 & 0.221 & 0.785 \\
fill+wkde & 0.0117 & 0.105 & 23.3 & $1.39 \mathrm{E}+05$ & $1.16 \mathrm{E}+08$ & $2.87 \mathrm{E}+15$ \\
\hline
\end{tabular}

Table 41: Error quantiles evaluating at the observed sample points

\begin{tabular}{lllllll}
\hline & $25 \%$ & $50 \%$ & $75 \%$ & $90 \%$ & $95 \%$ & $99 \%$ \\
\hline kde & 0.0308 & 0.0642 & 0.108 & 0.156 & 0.202 & 0.367 \\
wkde & 0.0304 & 0.0634 & 0.106 & 0.154 & 0.197 & 0.347 \\
wkdeA & 0.0303 & 0.0632 & 0.106 & 0.153 & 0.196 & 0.342 \\
fill+wkde & 0.0311 & 0.0647 & 0.108 & 0.154 & 0.194 & 0.343 \\
\hline
\end{tabular}


Case No. 21

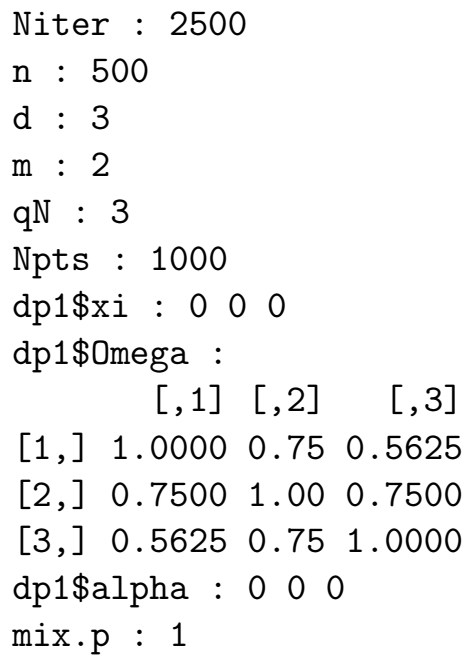

Table 42: Error quantiles for a fixed grid of points

\begin{tabular}{lllllll}
\hline & $25 \%$ & $50 \%$ & $75 \%$ & $90 \%$ & $95 \%$ & $99 \%$ \\
\hline kde & $9 \mathrm{E}-06$ & 0.00113 & 0.0174 & 0.0557 & 0.0893 & 0.194 \\
wkde & $8.21 \mathrm{E}-06$ & 0.000996 & 0.0153 & 0.0489 & 0.0784 & 0.164 \\
wkdeA & $8.28 \mathrm{E}-06$ & 0.00101 & 0.0155 & 0.049 & 0.078 & 0.161 \\
fill+wkde & 0.000244 & 0.00794 & 0.0425 & 0.138 & 0.354 & 10.8 \\
\hline
\end{tabular}

Table 43: Error quantiles evaluating at the observed sample points

\begin{tabular}{lllllll}
\hline & $25 \%$ & $50 \%$ & $75 \%$ & $90 \%$ & $95 \%$ & $99 \%$ \\
\hline kde & 0.0224 & 0.047 & 0.0803 & 0.119 & 0.154 & 0.278 \\
wkde & 0.0209 & 0.0439 & 0.0752 & 0.112 & 0.143 & 0.255 \\
wkdeA & 0.0207 & 0.0435 & 0.0744 & 0.11 & 0.14 & 0.25 \\
fill+wkde & 0.0214 & 0.0449 & 0.0761 & 0.111 & 0.139 & 0.25 \\
\hline
\end{tabular}


Case No. 22

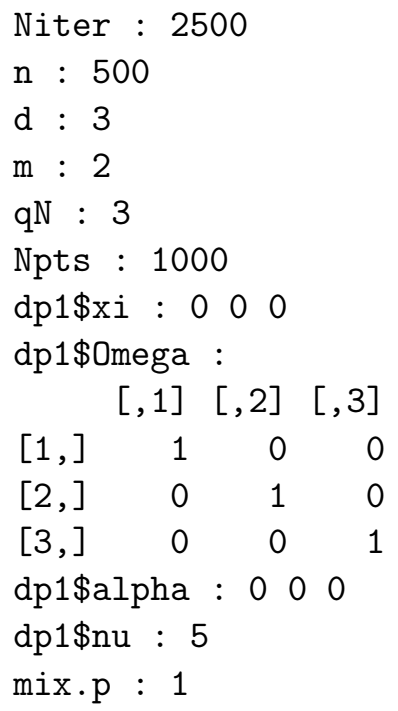

Table 44: Error quantiles for a fixed grid of points

\begin{tabular}{lllllll}
\hline & $25 \%$ & $50 \%$ & $75 \%$ & $90 \%$ & $95 \%$ & $99 \%$ \\
\hline kde & 0.00828 & 0.0143 & 0.0246 & 0.0434 & 0.0588 & 0.0937 \\
wkde & 0.00872 & 0.0147 & 0.0239 & 0.0399 & 0.0559 & 0.1 \\
wkdeA & 0.00853 & 0.0144 & 0.0235 & 0.0388 & 0.0538 & 0.0949 \\
fill+wkde & 0.0081 & 0.014 & 0.0232 & 0.0366 & 0.05 & 0.0867 \\
\hline
\end{tabular}

Table 45: Error quantiles evaluating at the observed sample points

\begin{tabular}{lllllll}
\hline & $25 \%$ & $50 \%$ & $75 \%$ & $90 \%$ & $95 \%$ & $99 \%$ \\
\hline kde & 0.0157 & 0.0329 & 0.0573 & 0.0914 & 0.139 & 0.474 \\
wkde & 0.0134 & 0.0288 & 0.0524 & 0.0858 & 0.125 & 0.454 \\
wkdeA & 0.0133 & 0.0285 & 0.0513 & 0.0832 & 0.122 & 0.448 \\
fill+wkde & 0.0107 & 0.0234 & 0.0434 & 0.07 & 0.0988 & 0.318 \\
\hline
\end{tabular}


Case No. 23

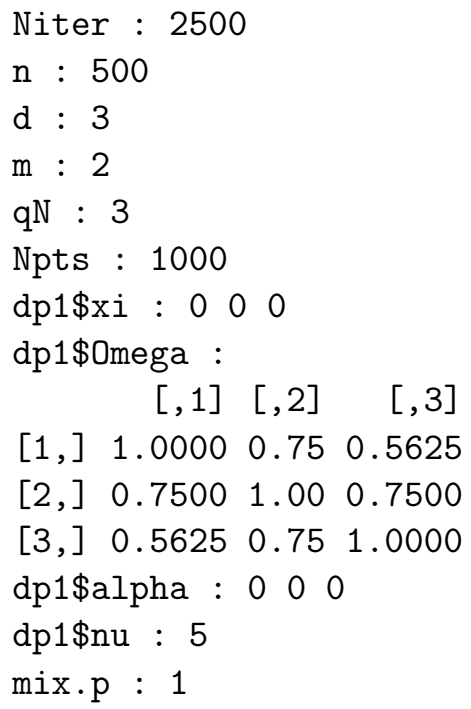

Table 46: Error quantiles for a fixed grid of points

\begin{tabular}{lllllll}
\hline & $25 \%$ & $50 \%$ & $75 \%$ & $90 \%$ & $95 \%$ & $99 \%$ \\
\hline kde & 0.00369 & 0.00857 & 0.0224 & 0.0532 & 0.0811 & 0.155 \\
wkde & 0.00373 & 0.00845 & 0.0194 & 0.0419 & 0.0652 & 0.126 \\
wkdeA & 0.00371 & 0.00834 & 0.0192 & 0.0415 & 0.064 & 0.122 \\
fill+wkde & 0.00354 & 0.00836 & 0.0221 & 0.0496 & 0.075 & 0.144 \\
\hline
\end{tabular}

Table 47: Error quantiles evaluating at the observed sample points

\begin{tabular}{lllllll}
\hline & $25 \%$ & $50 \%$ & $75 \%$ & $90 \%$ & $95 \%$ & $99 \%$ \\
\hline kde & 0.0275 & 0.0576 & 0.098 & 0.144 & 0.197 & 0.639 \\
wkde & 0.0229 & 0.0492 & 0.0863 & 0.131 & 0.181 & 0.61 \\
wkdeA & 0.0232 & 0.0492 & 0.0854 & 0.129 & 0.178 & 0.607 \\
fill+wkde & 0.024 & 0.0512 & 0.0894 & 0.132 & 0.167 & 0.517 \\
\hline
\end{tabular}


Case No. 24

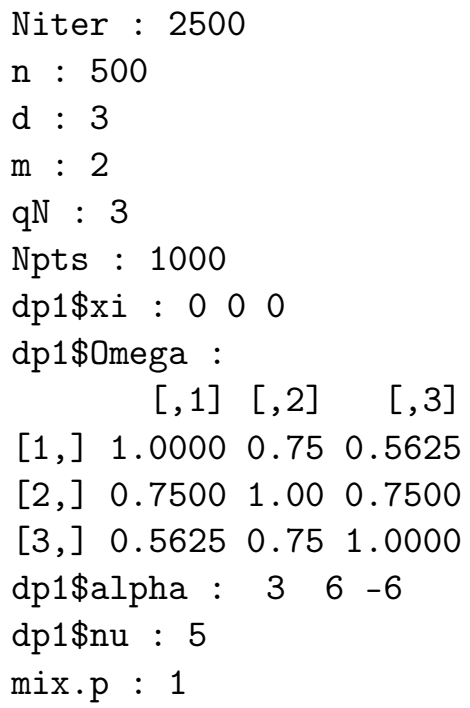

Table 48: Error quantiles for a fixed grid of points

\begin{tabular}{lllllll}
\hline & $25 \%$ & $50 \%$ & $75 \%$ & $90 \%$ & $95 \%$ & $99 \%$ \\
\hline kde & 0.000145 & 0.00468 & 0.0195 & 0.0651 & 0.126 & 0.422 \\
wkde & 0.000145 & 0.00462 & 0.0184 & 0.0546 & 0.109 & 0.388 \\
wkdeA & 0.000145 & 0.00461 & 0.0183 & 0.0543 & 0.108 & 0.386 \\
fill+wkde & 0.000524 & 0.00638 & 0.027 & 0.101 & 0.264 & 1.78 \\
\hline
\end{tabular}

Table 49: Error quantiles evaluating at the observed sample points

\begin{tabular}{lllllll}
\hline & $25 \%$ & $50 \%$ & $75 \%$ & $90 \%$ & $95 \%$ & $99 \%$ \\
\hline kde & 0.0363 & 0.0771 & 0.137 & 0.21 & 0.274 & 0.81 \\
wkde & 0.0311 & 0.0685 & 0.126 & 0.198 & 0.26 & 0.784 \\
wkdeA & 0.0311 & 0.0681 & 0.125 & 0.196 & 0.258 & 0.782 \\
fill+wkde & 0.0318 & 0.0702 & 0.131 & 0.205 & 0.256 & 0.689 \\
\hline
\end{tabular}




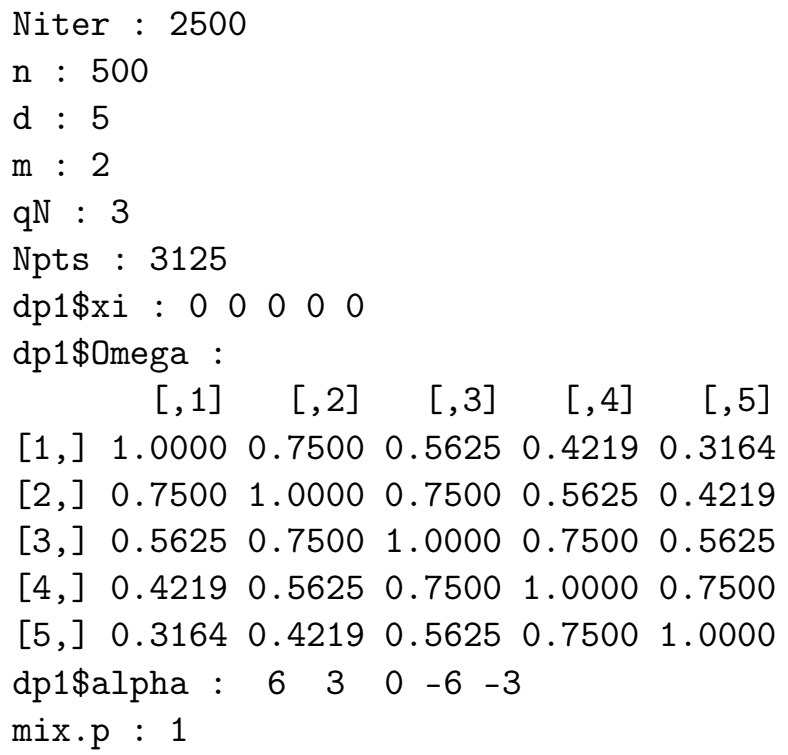

Table 50: Error quantiles for a fixed grid of points

\begin{tabular}{lllllll}
\hline & $25 \%$ & $50 \%$ & $75 \%$ & $90 \%$ & $95 \%$ & $99 \%$ \\
\hline kde & $4.95 \mathrm{E}-10$ & $1.9 \mathrm{E}-05$ & $1.29 \mathrm{E}+07$ & $1.84 \mathrm{E}+53$ & $2.87 \mathrm{E}+84$ & Inf \\
wkde & $4.16 \mathrm{E}-10$ & $1.39 \mathrm{E}-05$ & $6.14 \mathrm{E}+06$ & $5.23 \mathrm{E}+52$ & $1.83 \mathrm{E}+84$ & Inf \\
wkdeA & $4.35 \mathrm{E}-10$ & $1.52 \mathrm{E}-05$ & $7.75 \mathrm{E}+06$ & $8.53 \mathrm{E}+52$ & $2.11 \mathrm{E}+84$ & Inf \\
fill+wkde & 0.000446 & 0.143 & $6.02 \mathrm{E}+15$ & $2.72 \mathrm{E}+70$ & $5.5 \mathrm{E}+102$ & Inf \\
\hline
\end{tabular}

Table 51: Error quantiles evaluating at the observed sample points

\begin{tabular}{lllllll}
\hline & $25 \%$ & $50 \%$ & $75 \%$ & $90 \%$ & $95 \%$ & $99 \%$ \\
\hline kde & 0.031 & 0.0671 & 0.125 & 0.23 & 0.353 & 0.901 \\
wkde & 0.0253 & 0.0561 & 0.108 & 0.205 & 0.32 & 0.837 \\
wkdeA & 0.0262 & 0.0573 & 0.109 & 0.204 & 0.317 & 0.833 \\
fill+wkde & 0.0238 & 0.052 & 0.0959 & 0.16 & 0.245 & 0.65 \\
\hline
\end{tabular}




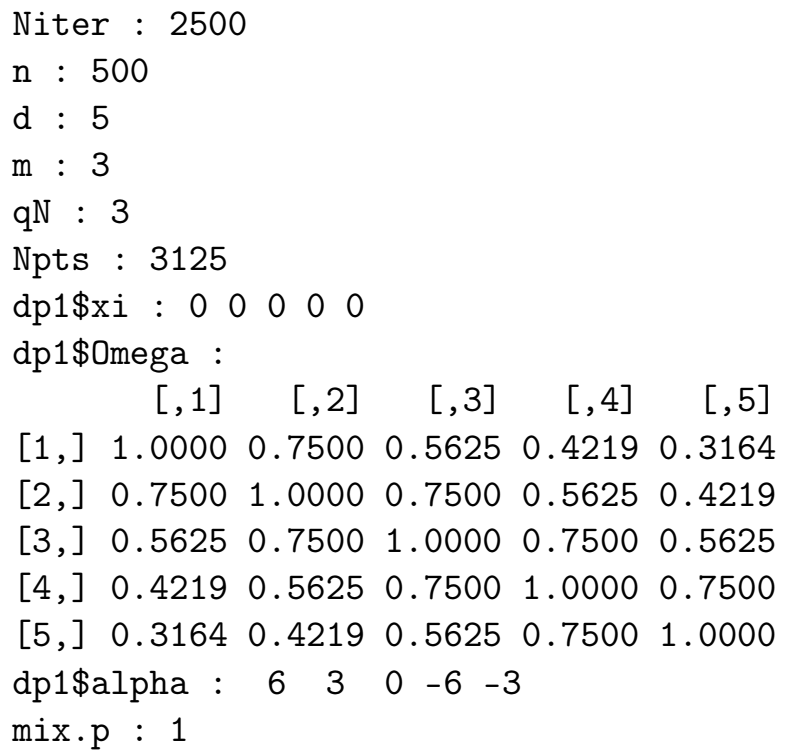

Table 52: Error quantiles for a fixed grid of points

\begin{tabular}{lllllll}
\hline & $25 \%$ & $50 \%$ & $75 \%$ & $90 \%$ & $95 \%$ & $99 \%$ \\
\hline kde & $4.73 \mathrm{E}-10$ & $1.78 \mathrm{E}-05$ & $9.03 \mathrm{E}+06$ & $6.8 \mathrm{E}+52$ & $1.45 \mathrm{E}+84$ & Inf \\
wkde & $4.6 \mathrm{E}-10$ & $1.69 \mathrm{E}-05$ & $8.12 \mathrm{E}+06$ & $6.19 \mathrm{E}+52$ & $1.27 \mathrm{E}+84$ & Inf \\
wkdeA & $4.66 \mathrm{E}-10$ & $1.74 \mathrm{E}-05$ & $8.65 \mathrm{E}+06$ & $6.6 \mathrm{E}+52$ & $1.38 \mathrm{E}+84$ & Inf \\
fill+wkde & $8.95 \mathrm{E}-08$ & 0.000937 & $5.11 \mathrm{E}+11$ & $8.65 \mathrm{E}+60$ & $1.23 \mathrm{E}+95$ & Inf \\
\hline
\end{tabular}

Table 53: Error quantiles evaluating at the observed sample points

\begin{tabular}{lllllll}
\hline & $25 \%$ & $50 \%$ & $75 \%$ & $90 \%$ & $95 \%$ & $99 \%$ \\
\hline kde & 0.031 & 0.0672 & 0.125 & 0.231 & 0.353 & 0.898 \\
wkde & 0.0296 & 0.0647 & 0.122 & 0.229 & 0.352 & 0.91 \\
wkdeA & 0.0302 & 0.0657 & 0.123 & 0.229 & 0.353 & 0.907 \\
fill+wkde & 0.0286 & 0.062 & 0.115 & 0.208 & 0.323 & 0.834 \\
\hline
\end{tabular}




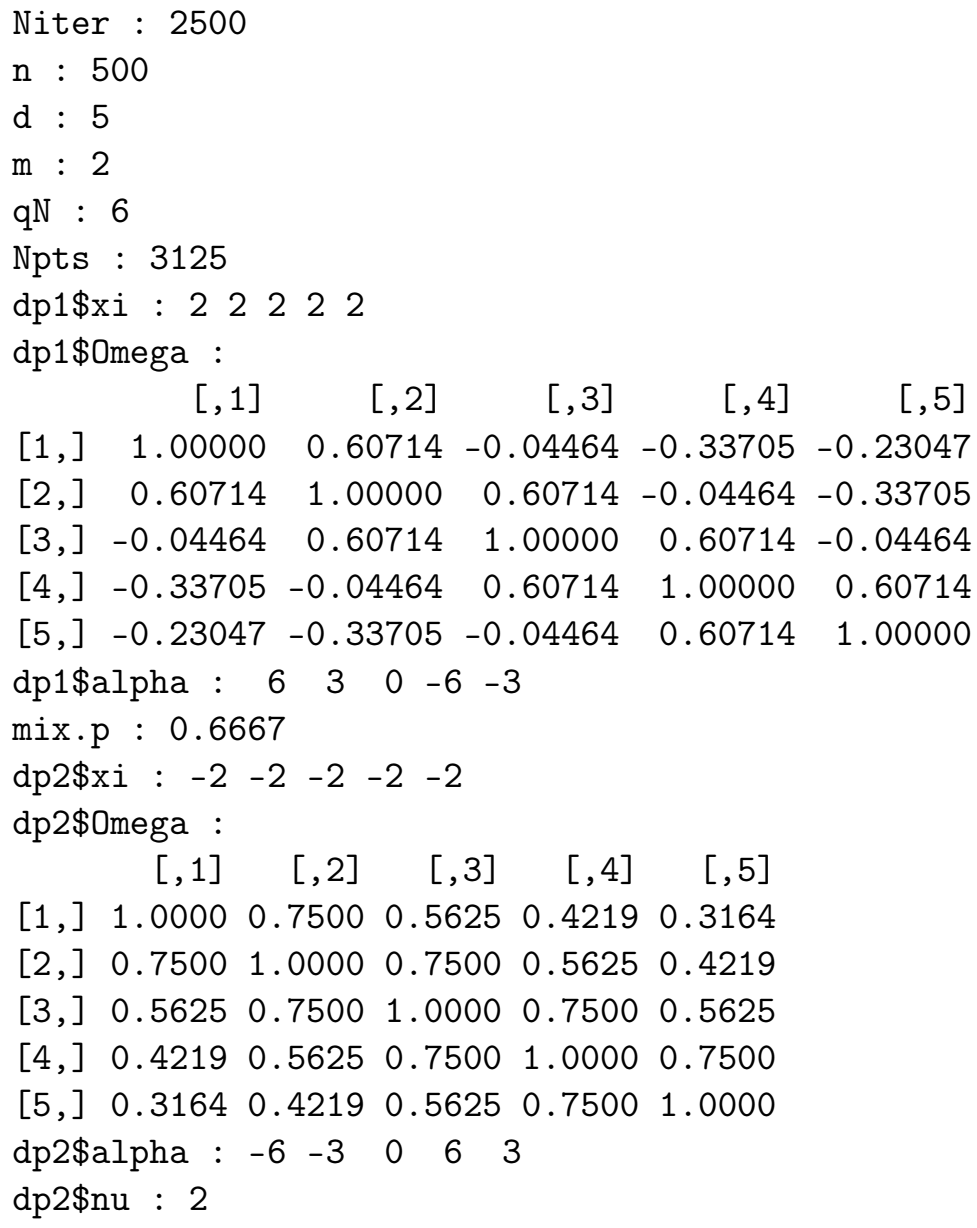

Table 54: Error quantiles for a fixed grid of points

\begin{tabular}{lllllll}
\hline & $25 \%$ & $50 \%$ & $75 \%$ & $90 \%$ & $95 \%$ & $99 \%$ \\
\hline kde & $3.31 \mathrm{E}-06$ & $3.14 \mathrm{E}-05$ & 0.000159 & 0.00115 & 0.00416 & 0.0373 \\
wkde & $3.72 \mathrm{E}-06$ & $3.37 \mathrm{E}-05$ & 0.000163 & 0.00113 & 0.00361 & 0.0272 \\
wkdeA & $3.7 \mathrm{E}-06$ & $3.39 \mathrm{E}-05$ & 0.000163 & 0.00113 & 0.00359 & 0.0273 \\
fill+wkde & $3.4 \mathrm{E}-06$ & $3.24 \mathrm{E}-05$ & 0.000162 & 0.0012 & 0.0042 & 0.0742 \\
\hline
\end{tabular}

Table 55: Error quantiles evaluating at the observed sample points

\begin{tabular}{lllllll}
\hline & $25 \%$ & $50 \%$ & $75 \%$ & $90 \%$ & $95 \%$ & $99 \%$ \\
\hline kde & 0.0274 & 0.0619 & 0.115 & 0.176 & 0.249 & 3.42 \\
wkde & 0.0288 & 0.0638 & 0.116 & 0.174 & 0.223 & 3.57 \\
wkdeA & 0.0295 & 0.0643 & 0.116 & 0.174 & 0.226 & 3.66 \\
fill+wkde & 0.026 & 0.0619 & 0.115 & 0.17 & 0.208 & 1.74 \\
\hline
\end{tabular}




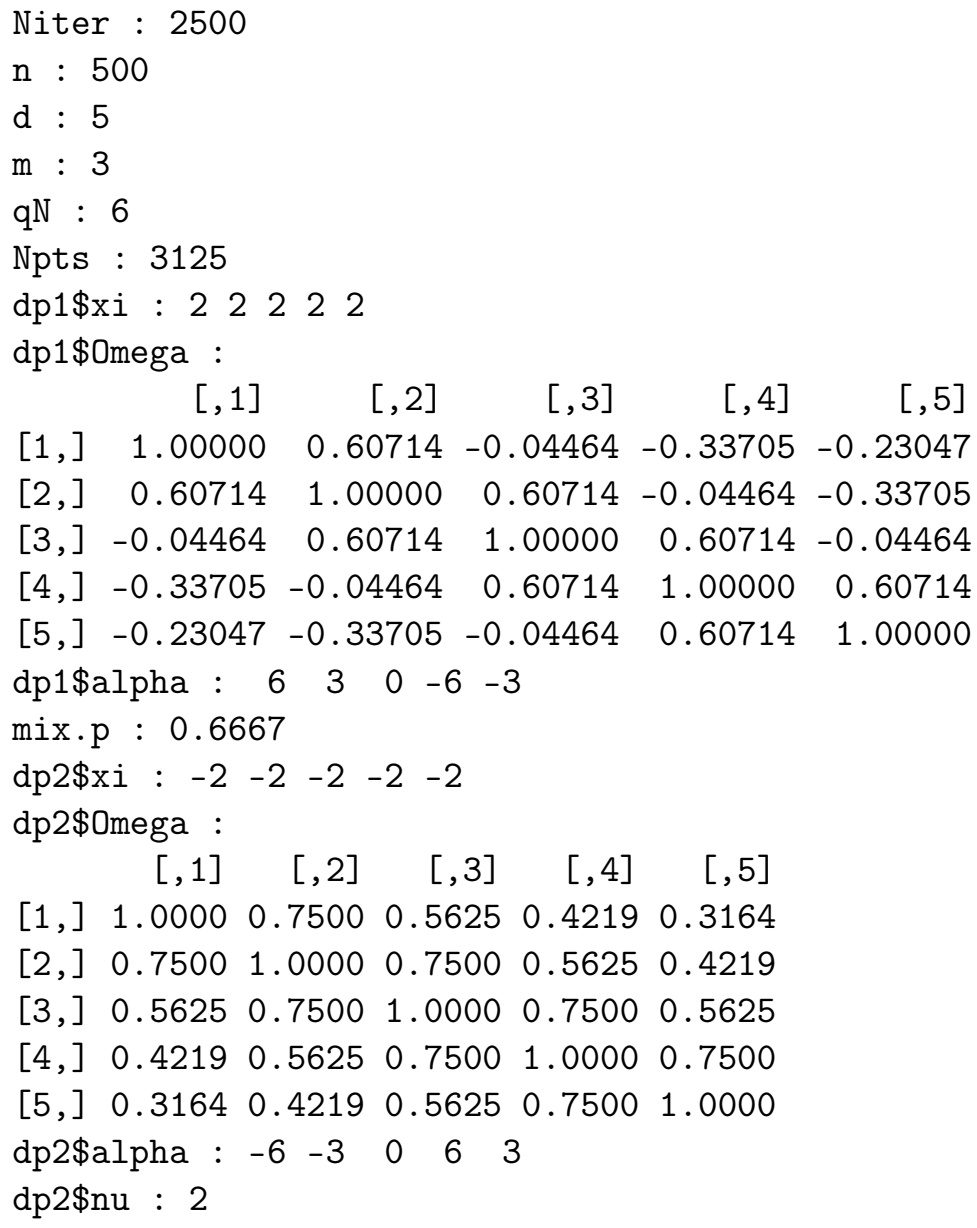

Table 56: Error quantiles for a fixed grid of points

\begin{tabular}{lllllll}
\hline & $25 \%$ & $50 \%$ & $75 \%$ & $90 \%$ & $95 \%$ & $99 \%$ \\
\hline kde & $3.44 \mathrm{E}-06$ & $3.15 \mathrm{E}-05$ & 0.000149 & 0.00099 & 0.00317 & 0.0243 \\
wkde & $3.43 \mathrm{E}-06$ & $3.15 \mathrm{E}-05$ & 0.000149 & 0.000987 & 0.00316 & 0.0245 \\
wkdeA & $3.43 \mathrm{E}-06$ & $3.15 \mathrm{E}-05$ & 0.000149 & 0.000987 & 0.00316 & 0.0245 \\
fill+wkde & $3.47 \mathrm{E}-06$ & $3.19 \mathrm{E}-05$ & 0.00015 & 0.000996 & 0.00319 & 0.0269 \\
\hline
\end{tabular}

Table 57: Error quantiles evaluating at the observed sample points

\begin{tabular}{lllllll}
\hline & $25 \%$ & $50 \%$ & $75 \%$ & $90 \%$ & $95 \%$ & $99 \%$ \\
\hline kde & 0.0275 & 0.0621 & 0.115 & 0.176 & 0.25 & 3.34 \\
wkde & 0.0277 & 0.0625 & 0.116 & 0.177 & 0.253 & 3.46 \\
wkdeA & 0.0277 & 0.0625 & 0.116 & 0.177 & 0.253 & 3.46 \\
fill+wkde & 0.0275 & 0.0624 & 0.116 & 0.177 & 0.247 & 3.32 \\
\hline
\end{tabular}




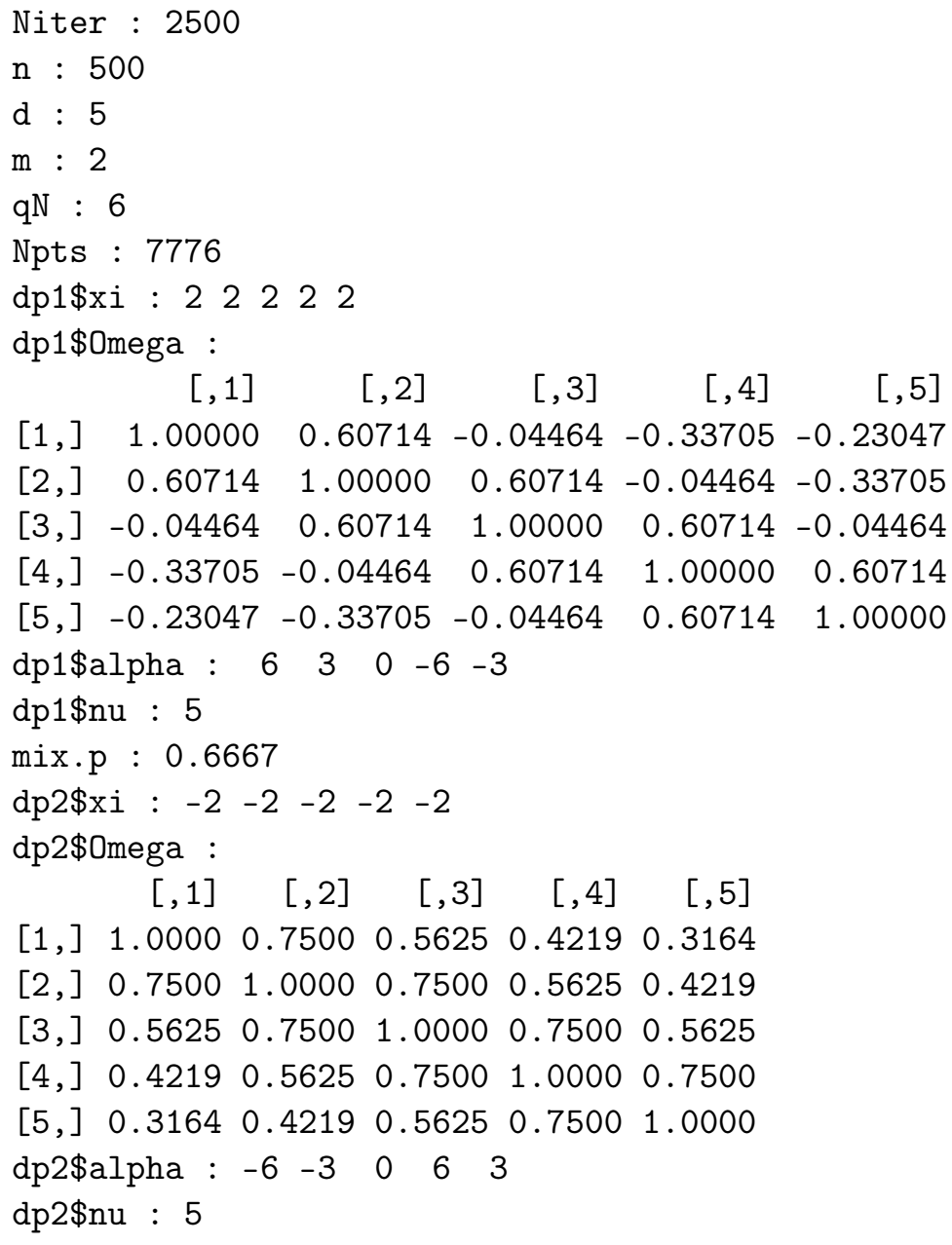

Table 58: Error quantiles for a fixed grid of points

\begin{tabular}{lllllll}
\hline & $25 \%$ & $50 \%$ & $75 \%$ & $90 \%$ & $95 \%$ & $99 \%$ \\
\hline kde & $3.61 \mathrm{E}-06$ & $1.26 \mathrm{E}-05$ & $4.88 \mathrm{E}-05$ & 0.000173 & 0.000381 & 0.00405 \\
wkde & $3.61 \mathrm{E}-06$ & $1.26 \mathrm{E}-05$ & $4.88 \mathrm{E}-05$ & 0.000173 & 0.000376 & 0.00349 \\
wkdeA & $3.61 \mathrm{E}-06$ & $1.26 \mathrm{E}-05$ & $4.88 \mathrm{E}-05$ & 0.000173 & 0.000377 & 0.00359 \\
fill+wkde & $3.68 \mathrm{E}-06$ & $1.32 \mathrm{E}-05$ & $5.59 \mathrm{E}-05$ & 0.000281 & 0.00115 & 0.0201 \\
\hline
\end{tabular}

Table 59: Error quantiles evaluating at the observed sample points

\begin{tabular}{lllllll}
\hline & $25 \%$ & $50 \%$ & $75 \%$ & $90 \%$ & $95 \%$ & $99 \%$ \\
\hline kde & 0.0231 & 0.0531 & 0.107 & 0.184 & 0.248 & 0.889 \\
wkde & 0.0215 & 0.05 & 0.102 & 0.174 & 0.23 & 0.616 \\
wkdeA & 0.0222 & 0.0506 & 0.102 & 0.174 & 0.231 & 0.629 \\
fill+wkde & 0.021 & 0.0494 & 0.102 & 0.173 & 0.228 & 0.548 \\
\hline
\end{tabular}




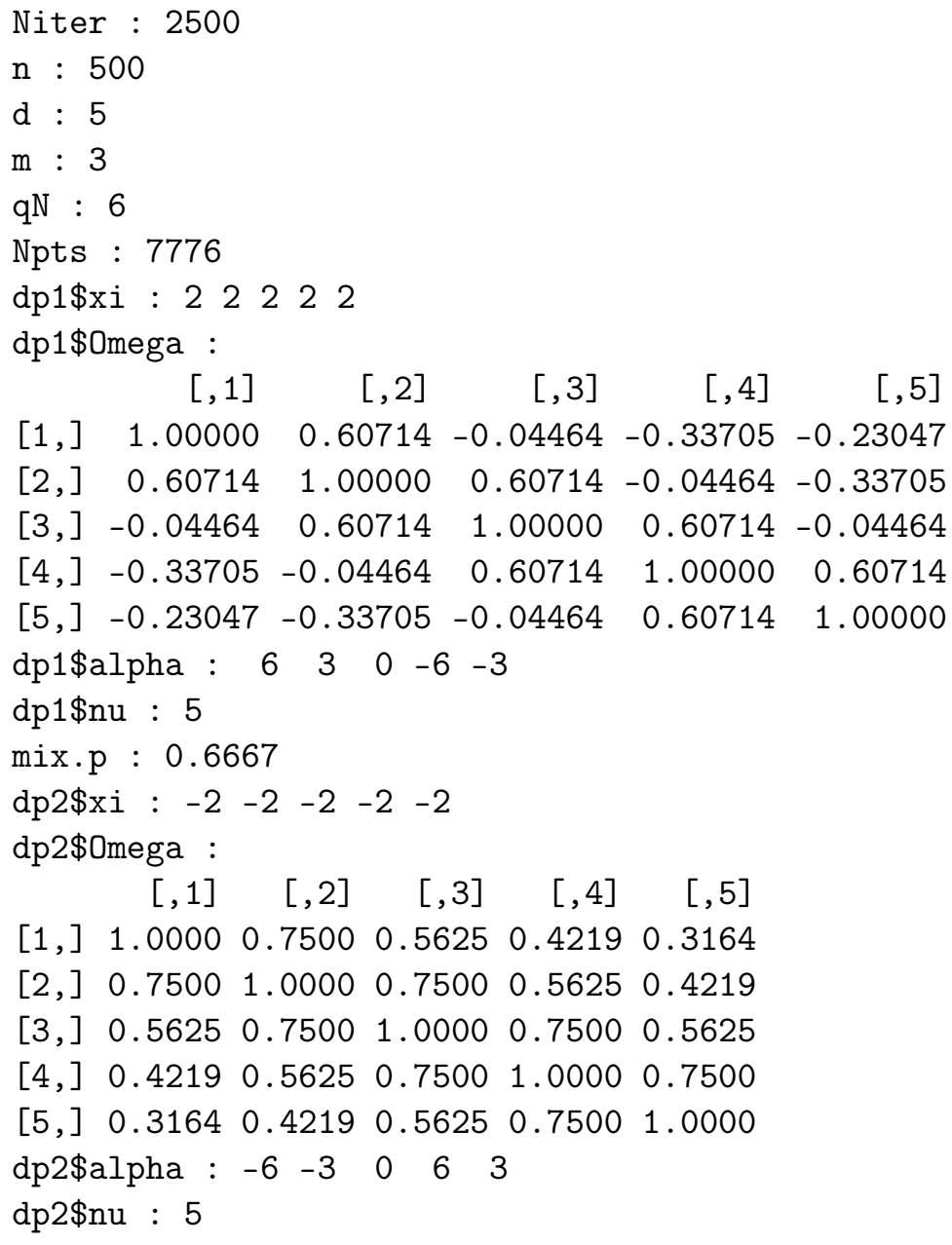

Table 60: Error quantiles for a fixed grid of points

\begin{tabular}{lllllll}
\hline & $25 \%$ & $50 \%$ & $75 \%$ & $90 \%$ & $95 \%$ & $99 \%$ \\
\hline kde & $3.61 \mathrm{E}-06$ & $1.26 \mathrm{E}-05$ & $4.88 \mathrm{E}-05$ & 0.000173 & 0.000381 & 0.00403 \\
wkde & $3.61 \mathrm{E}-06$ & $1.26 \mathrm{E}-05$ & $4.88 \mathrm{E}-05$ & 0.000173 & 0.000381 & 0.004 \\
wkdeA & $3.61 \mathrm{E}-06$ & $1.26 \mathrm{E}-05$ & $4.88 \mathrm{E}-05$ & 0.000173 & 0.000381 & 0.00401 \\
fill+wkde & $3.61 \mathrm{E}-06$ & $1.27 \mathrm{E}-05$ & $5 \mathrm{E}-05$ & 0.00019 & 0.000471 & 0.00742 \\
\hline
\end{tabular}

Table 61: Error quantiles evaluating at the observed sample points

\begin{tabular}{lllllll}
\hline & $25 \%$ & $50 \%$ & $75 \%$ & $90 \%$ & $95 \%$ & $99 \%$ \\
\hline kde & 0.0232 & 0.0532 & 0.108 & 0.184 & 0.248 & 0.869 \\
wkde & 0.0231 & 0.0529 & 0.107 & 0.183 & 0.247 & 0.865 \\
wkdeA & 0.0232 & 0.0531 & 0.107 & 0.183 & 0.247 & 0.868 \\
fill+wkde & 0.0229 & 0.0528 & 0.107 & 0.183 & 0.246 & 0.844 \\
\hline
\end{tabular}




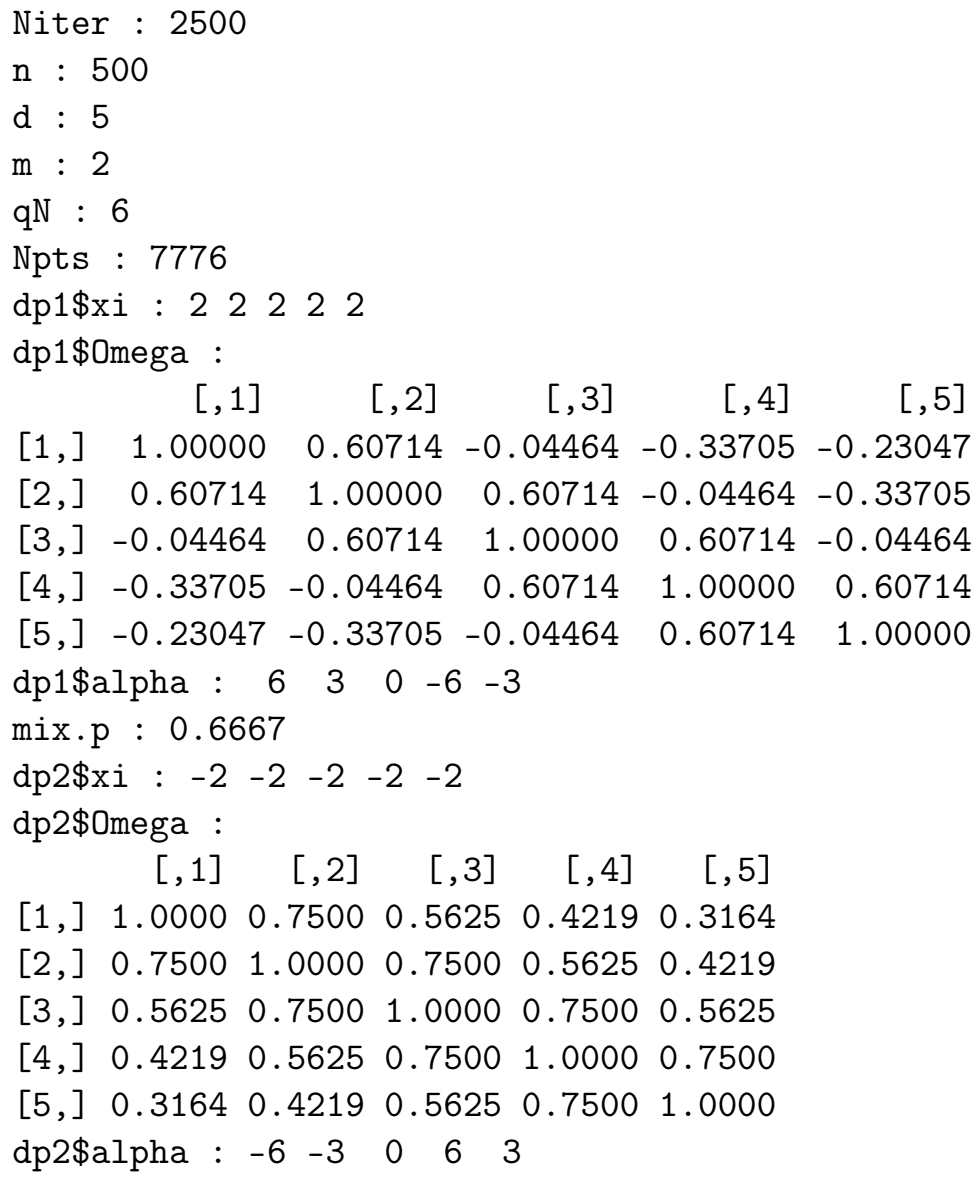

Table 62: Error quantiles for a fixed grid of points

\begin{tabular}{lllllll}
\hline & $25 \%$ & $50 \%$ & $75 \%$ & $90 \%$ & $95 \%$ & $99 \%$ \\
\hline kde & $3.83 \mathrm{E}-21$ & $1.02 \mathrm{E}-11$ & 0.00701 & $5.73 \mathrm{E}+18$ & $8.16 \mathrm{E}+41$ & Inf \\
wkde & $3.54 \mathrm{E}-21$ & $9.36 \mathrm{E}-12$ & 0.0064 & $5.27 \mathrm{E}+18$ & $6.29 \mathrm{E}+41$ & Inf \\
wkdeA & $3.69 \mathrm{E}-21$ & $9.87 \mathrm{E}-12$ & 0.0067 & $5.8 \mathrm{E}+18$ & $1 \mathrm{E}+42$ & Inf \\
fill+wkde & $5.42 \mathrm{E}-11$ & 0.0174 & $1.67 \mathrm{E}+11$ & $1.76 \mathrm{E}+37$ & $1.27 \mathrm{E}+65$ & Inf \\
\hline
\end{tabular}

Table 63: Error quantiles evaluating at the observed sample points

\begin{tabular}{lllllll}
\hline & $25 \%$ & $50 \%$ & $75 \%$ & $90 \%$ & $95 \%$ & $99 \%$ \\
\hline kde & 0.0229 & 0.0501 & 0.0902 & 0.135 & 0.164 & 0.226 \\
wkde & 0.0226 & 0.0497 & 0.0896 & 0.135 & 0.163 & 0.222 \\
wkdeA & 0.0228 & 0.05 & 0.0901 & 0.135 & 0.164 & 0.227 \\
fill+wkde & 0.0227 & 0.05 & 0.0901 & 0.135 & 0.164 & 0.221 \\
\hline
\end{tabular}




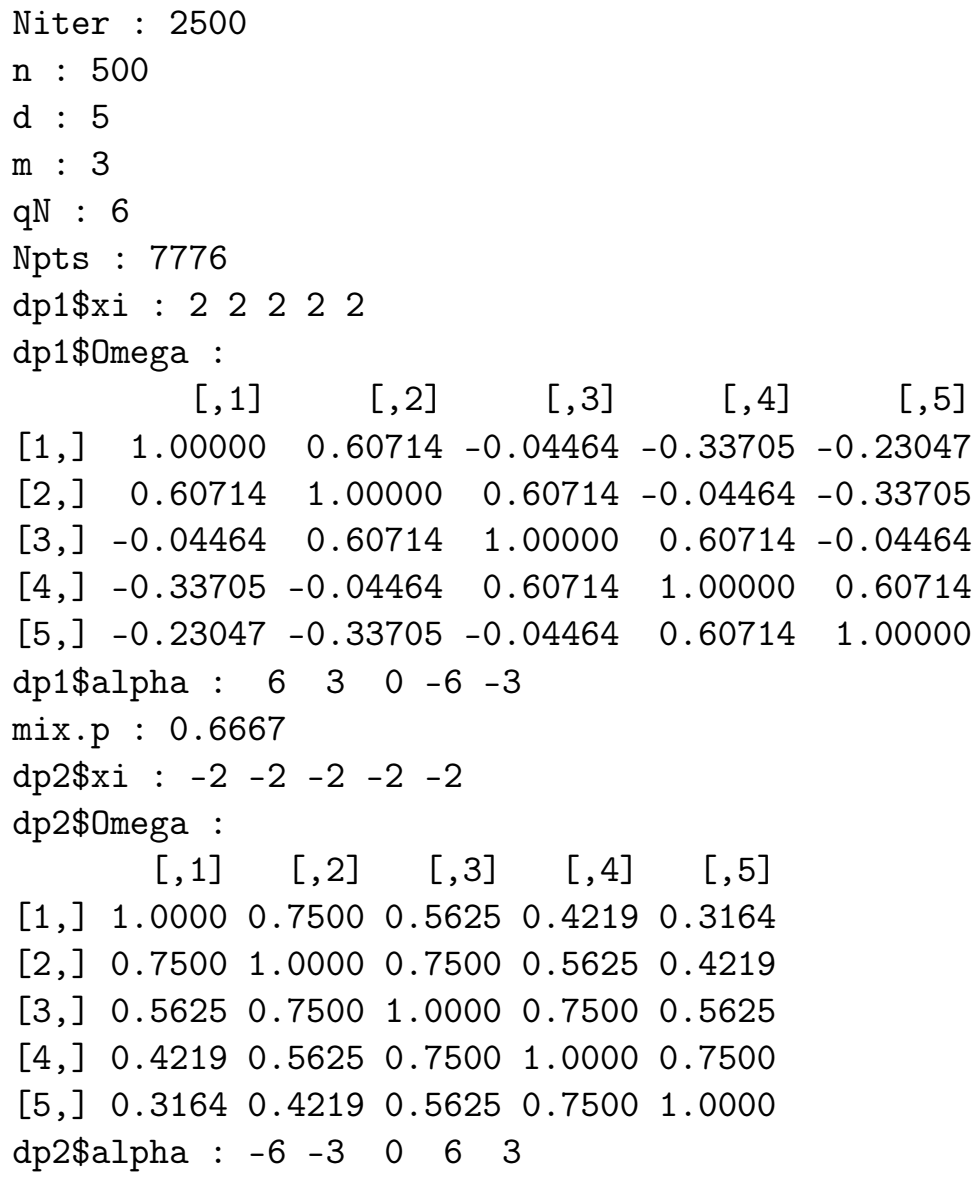

Table 64: Error quantiles for a fixed grid of points

\begin{tabular}{lllllll}
\hline & $25 \%$ & $50 \%$ & $75 \%$ & $90 \%$ & $95 \%$ & $99 \%$ \\
\hline kde & $2.94 \mathrm{E}-21$ & $9.36 \mathrm{E}-12$ & 0.00714 & $4.98 \mathrm{E}+18$ & $5.1 \mathrm{E}+41$ & Inf \\
wkde & $2.89 \mathrm{E}-21$ & $9.29 \mathrm{E}-12$ & 0.00707 & $4.93 \mathrm{E}+18$ & $4.93 \mathrm{E}+41$ & Inf \\
wkdeA & $2.93 \mathrm{E}-21$ & $9.33 \mathrm{E}-12$ & 0.00711 & $4.89 \mathrm{E}+18$ & $4.98 \mathrm{E}+41$ & Inf \\
fill+wkde & $3.05 \mathrm{E}-16$ & $5.17 \mathrm{E}-07$ & $1.99 \mathrm{E}+04$ & $1.01 \mathrm{E}+28$ & $1.47 \mathrm{E}+55$ & Inf \\
\hline
\end{tabular}

Table 65: Error quantiles evaluating at the observed sample points

\begin{tabular}{lllllll}
\hline & $25 \%$ & $50 \%$ & $75 \%$ & $90 \%$ & $95 \%$ & $99 \%$ \\
\hline kde & 0.0229 & 0.0501 & 0.0903 & 0.135 & 0.164 & 0.227 \\
wkde & 0.0229 & 0.0501 & 0.0903 & 0.135 & 0.164 & 0.227 \\
wkdeA & 0.0229 & 0.05 & 0.0903 & 0.135 & 0.164 & 0.227 \\
fill+wkde & 0.0229 & 0.0501 & 0.0904 & 0.136 & 0.164 & 0.226 \\
\hline
\end{tabular}




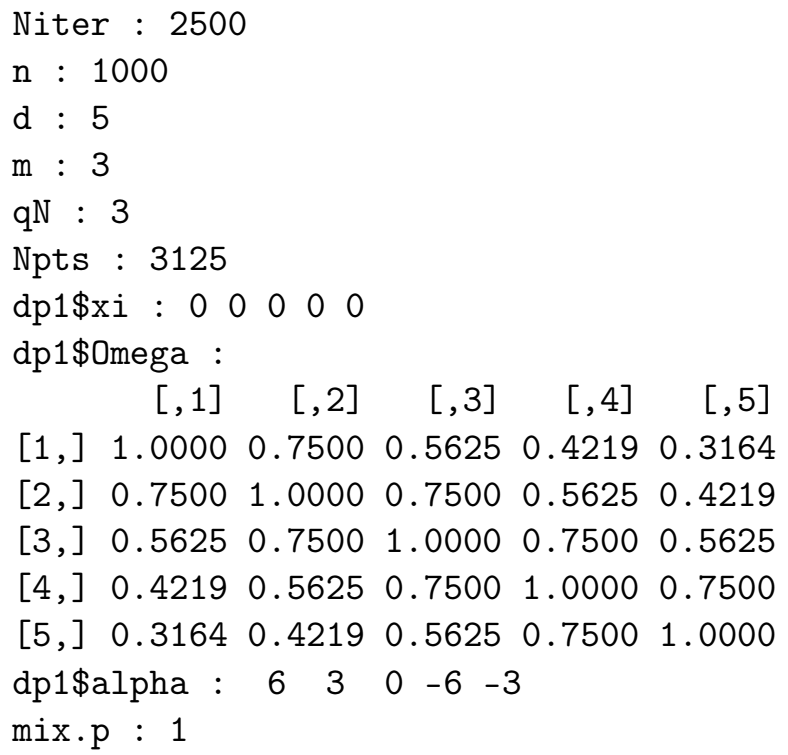

Table 66: Error quantiles for a fixed grid of points

\begin{tabular}{lllllll}
\hline & $25 \%$ & $50 \%$ & $75 \%$ & $90 \%$ & $95 \%$ & $99 \%$ \\
\hline kde & $1.79 \mathrm{E}-10$ & $5.7 \mathrm{E}-06$ & $1.02 \mathrm{E}+04$ & $3.89 \mathrm{E}+46$ & $3.37 \mathrm{E}+78$ & Inf \\
wkde & $1.73 \mathrm{E}-10$ & $5.59 \mathrm{E}-06$ & $9.04 \mathrm{E}+03$ & $3.35 \mathrm{E}+46$ & $3.01 \mathrm{E}+78$ & Inf \\
wkdeA & $1.76 \mathrm{E}-10$ & $5.64 \mathrm{E}-06$ & $9.62 \mathrm{E}+03$ & $3.51 \mathrm{E}+46$ & $3.11 \mathrm{E}+78$ & Inf \\
fill + wkde & $1.37 \mathrm{E}-08$ & 0.000521 & $7.61 \mathrm{E}+09$ & $2.95 \mathrm{E}+56$ & $2.3 \mathrm{E}+92$ & Inf \\
\hline
\end{tabular}

Table 67: Error quantiles evaluating at the observed sample points

\begin{tabular}{lllllll}
\hline & $25 \%$ & $50 \%$ & $75 \%$ & $90 \%$ & $95 \%$ & $99 \%$ \\
\hline kde & 0.0267 & 0.0578 & 0.106 & 0.188 & 0.287 & 0.728 \\
wkde & 0.0245 & 0.0536 & 0.101 & 0.185 & 0.285 & 0.744 \\
wkdeA & 0.0251 & 0.0545 & 0.102 & 0.184 & 0.284 & 0.738 \\
fill+wkde & 0.0236 & 0.0514 & 0.0944 & 0.162 & 0.251 & 0.657 \\
\hline
\end{tabular}




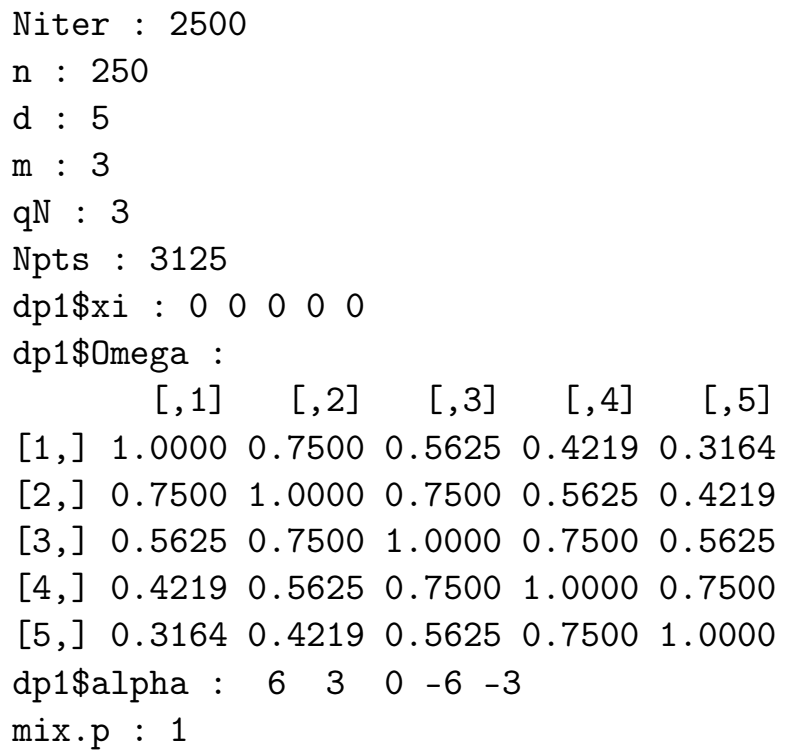

Table 68: Error quantiles for a fixed grid of points

\begin{tabular}{lllllll}
\hline & $25 \%$ & $50 \%$ & $75 \%$ & $90 \%$ & $95 \%$ & $99 \%$ \\
\hline kde & $3.71 \mathrm{E}-09$ & $8.1 \mathrm{E}-05$ & $1.58 \mathrm{E}+10$ & $4.49 \mathrm{E}+58$ & $1.18 \mathrm{E}+89$ & Inf \\
wkde & $3.58 \mathrm{E}-09$ & $7.9 \mathrm{E}-05$ & $1.51 \mathrm{E}+10$ & $4.04 \mathrm{E}+58$ & $1.14 \mathrm{E}+89$ & Inf \\
wkdeA & $3.65 \mathrm{E}-09$ & $8.01 \mathrm{E}-05$ & $1.53 \mathrm{E}+10$ & $4.28 \mathrm{E}+58$ & $1.14 \mathrm{E}+89$ & Inf \\
fill+wkde & $5.88 \mathrm{E}-07$ & 0.00162 & $1.1 \mathrm{E}+13$ & $5.75 \mathrm{E}+64$ & $1.33 \mathrm{E}+98$ & Inf \\
\hline
\end{tabular}

Table 69: Error quantiles evaluating at the observed sample points

\begin{tabular}{lllllll}
\hline & $25 \%$ & $50 \%$ & $75 \%$ & $90 \%$ & $95 \%$ & $99 \%$ \\
\hline kde & 0.0361 & 0.0783 & 0.149 & 0.284 & 0.435 & 1.09 \\
wkde & 0.0355 & 0.0773 & 0.148 & 0.285 & 0.438 & 1.11 \\
wkdeA & 0.036 & 0.0779 & 0.149 & 0.285 & 0.437 & 1.11 \\
fill+wkde & 0.0345 & 0.0748 & 0.141 & 0.268 & 0.414 & 1.05 \\
\hline
\end{tabular}




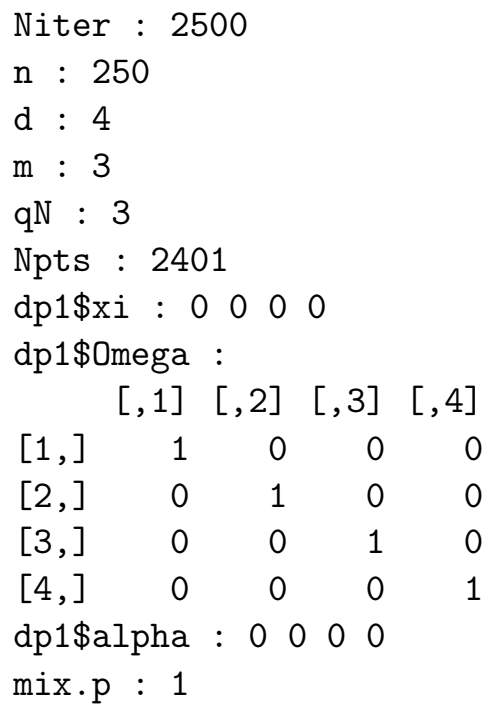

Table 70: Error quantiles for a fixed grid of points

\begin{tabular}{lllllll}
\hline & $25 \%$ & $50 \%$ & $75 \%$ & $90 \%$ & $95 \%$ & $99 \%$ \\
\hline kde & 0.000683 & 0.00247 & 0.00828 & 0.0239 & 0.0424 & 0.119 \\
wkde & 0.000686 & 0.00248 & 0.00826 & 0.0236 & 0.0417 & 0.119 \\
wkdeA & 0.000684 & 0.00247 & 0.00828 & 0.0238 & 0.0422 & 0.119 \\
fill+wkde & 0.000698 & 0.00282 & 0.0098 & 0.0288 & 0.0502 & 0.134 \\
\hline
\end{tabular}

Table 71: Error quantiles evaluating at the observed sample points

\begin{tabular}{lllllll}
\hline & $25 \%$ & $50 \%$ & $75 \%$ & $90 \%$ & $95 \%$ & $99 \%$ \\
\hline kde & 0.0222 & 0.049 & 0.0943 & 0.165 & 0.243 & 0.579 \\
wkde & 0.022 & 0.049 & 0.0951 & 0.167 & 0.245 & 0.582 \\
wkdeA & 0.0222 & 0.0494 & 0.0949 & 0.166 & 0.243 & 0.578 \\
fill+wkde & 0.0184 & 0.041 & 0.0825 & 0.15 & 0.221 & 0.522 \\
\hline
\end{tabular}




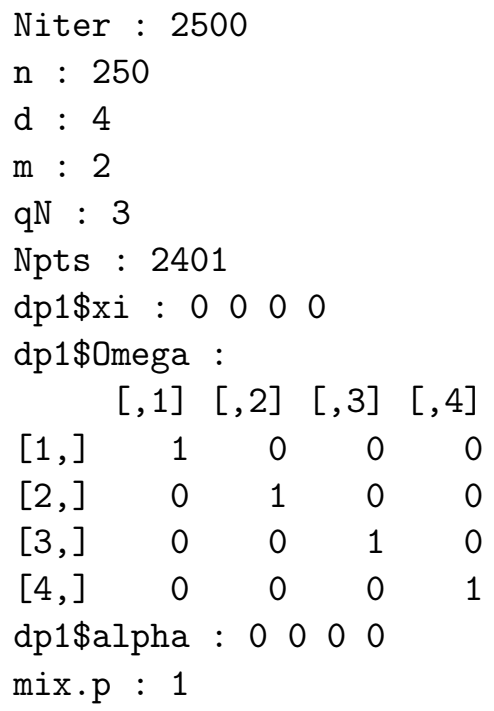

Table 72: Error quantiles for a fixed grid of points

\begin{tabular}{lllllll}
\hline & $25 \%$ & $50 \%$ & $75 \%$ & $90 \%$ & $95 \%$ & $99 \%$ \\
\hline kde & 0.000685 & 0.00247 & 0.00826 & 0.0238 & 0.0423 & 0.119 \\
wkde & 0.000691 & 0.00247 & 0.00807 & 0.0224 & 0.0388 & 0.11 \\
wkdeA & 0.000687 & 0.00245 & 0.00808 & 0.0225 & 0.0391 & 0.109 \\
fill+wkde & 0.000857 & 0.00372 & 0.014 & 0.0398 & 0.0674 & 0.176 \\
\hline
\end{tabular}

Table 73: Error quantiles evaluating at the observed sample points

\begin{tabular}{lllllll}
\hline & $25 \%$ & $50 \%$ & $75 \%$ & $90 \%$ & $95 \%$ & $99 \%$ \\
\hline kde & 0.0225 & 0.0493 & 0.0941 & 0.165 & 0.243 & 0.586 \\
wkde & 0.0216 & 0.0478 & 0.0909 & 0.157 & 0.229 & 0.542 \\
wkdeA & 0.0226 & 0.0486 & 0.0899 & 0.154 & 0.225 & 0.535 \\
fill+wkde & 0.0145 & 0.0312 & 0.0595 & 0.112 & 0.168 & 0.39 \\
\hline
\end{tabular}




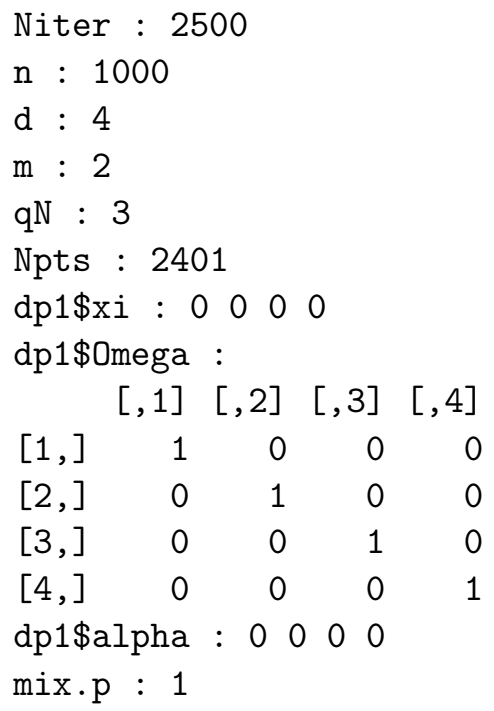

Table 74: Error quantiles for a fixed grid of points

\begin{tabular}{lllllll}
\hline & $25 \%$ & $50 \%$ & $75 \%$ & $90 \%$ & $95 \%$ & $99 \%$ \\
\hline kde & 0.000652 & 0.00225 & 0.00714 & 0.019 & 0.0321 & 0.0787 \\
wkde & 0.000654 & 0.00224 & 0.00676 & 0.0164 & 0.0268 & 0.0644 \\
wkdeA & 0.000653 & 0.00224 & 0.00673 & 0.0164 & 0.0268 & 0.0637 \\
fill+wkde & 0.00225 & 0.00766 & 0.0214 & 0.0467 & 0.0718 & 0.149 \\
\hline
\end{tabular}

Table 75: Error quantiles evaluating at the observed sample points

\begin{tabular}{lllllll}
\hline & $25 \%$ & $50 \%$ & $75 \%$ & $90 \%$ & $95 \%$ & $99 \%$ \\
\hline kde & 0.0111 & 0.024 & 0.045 & 0.0779 & 0.112 & 0.262 \\
wkde & 0.0108 & 0.0235 & 0.0433 & 0.0699 & 0.095 & 0.209 \\
wkdeA & 0.0107 & 0.023 & 0.0414 & 0.0667 & 0.0922 & 0.208 \\
fill+wkde & 0.00844 & 0.0177 & 0.0303 & 0.0491 & 0.0729 & 0.156 \\
\hline
\end{tabular}




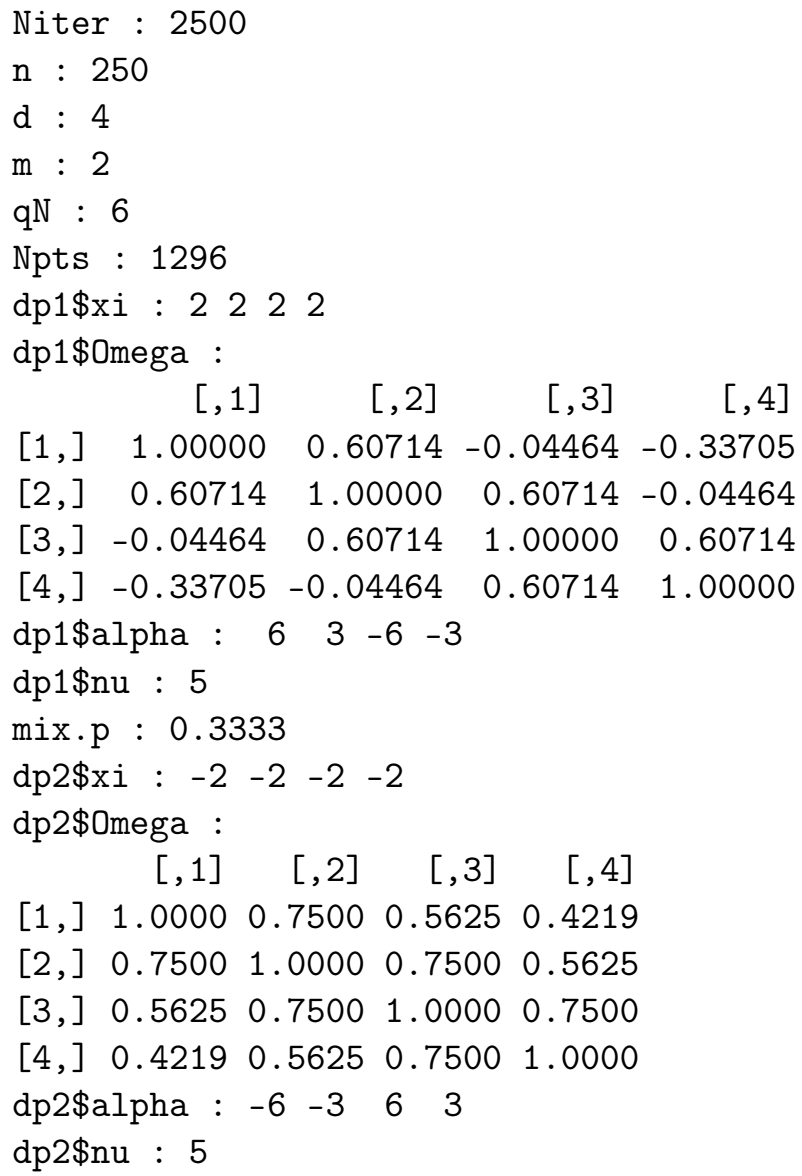

Table 76: Error quantiles for a fixed grid of points

\begin{tabular}{lllllll}
\hline & $25 \%$ & $50 \%$ & $75 \%$ & $90 \%$ & $95 \%$ & $99 \%$ \\
\hline kde & $2.89 \mathrm{E}-05$ & $9.71 \mathrm{E}-05$ & 0.000349 & 0.00119 & 0.00318 & 0.0473 \\
wkde & $2.89 \mathrm{E}-05$ & $9.71 \mathrm{E}-05$ & 0.000349 & 0.00118 & 0.00307 & 0.0445 \\
wkdeA & $2.89 \mathrm{E}-05$ & $9.71 \mathrm{E}-05$ & 0.000349 & 0.00118 & 0.00309 & 0.0446 \\
fill+wkde & $2.9 \mathrm{E}-05$ & $9.93 \mathrm{E}-05$ & 0.000372 & 0.00168 & 0.00613 & 0.0651 \\
\hline
\end{tabular}

Table 77: Error quantiles evaluating at the observed sample points

\begin{tabular}{lllllll}
\hline & $25 \%$ & $50 \%$ & $75 \%$ & $90 \%$ & $95 \%$ & $99 \%$ \\
\hline kde & 0.0309 & 0.0677 & 0.127 & 0.199 & 0.25 & 0.708 \\
wkde & 0.0297 & 0.0654 & 0.124 & 0.194 & 0.243 & 0.595 \\
wkdeA & 0.0301 & 0.0658 & 0.124 & 0.194 & 0.243 & 0.605 \\
fill+wkde & 0.0295 & 0.0652 & 0.124 & 0.194 & 0.242 & 0.569 \\
\hline
\end{tabular}




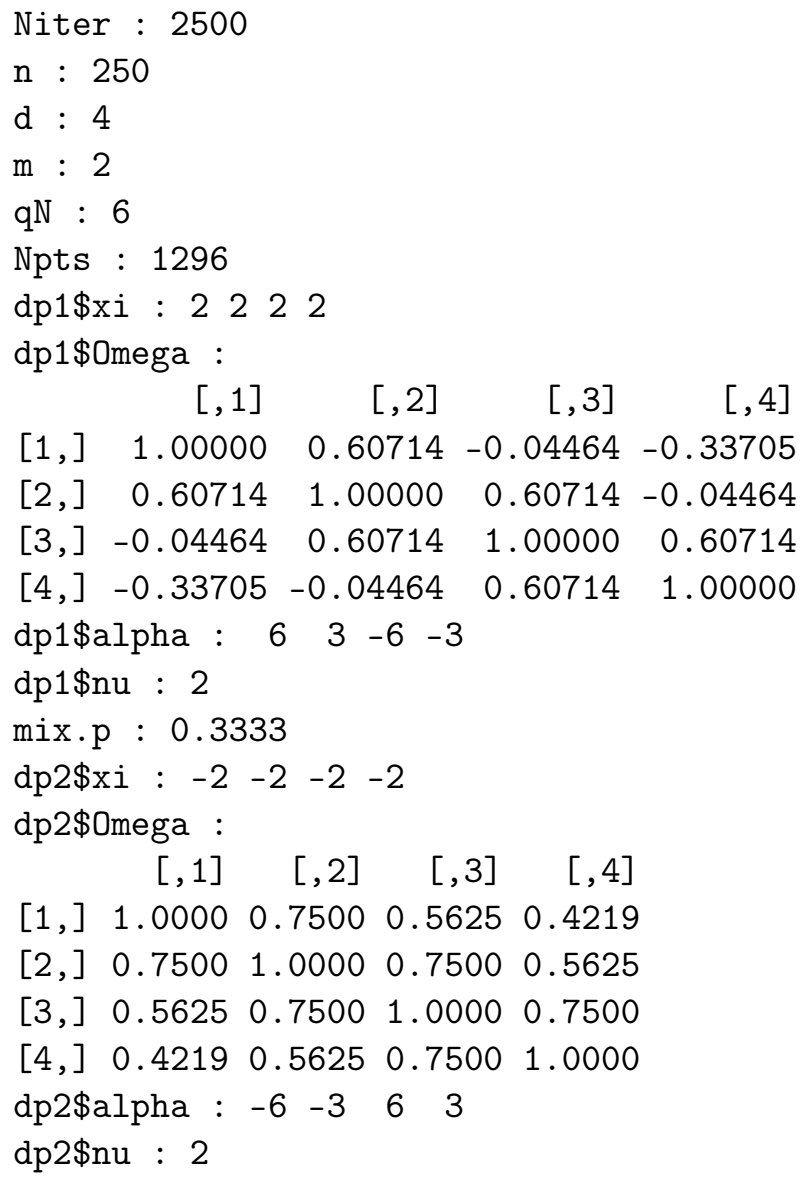

Table 78: Error quantiles for a fixed grid of points

\begin{tabular}{lllllll}
\hline & $25 \%$ & $50 \%$ & $75 \%$ & $90 \%$ & $95 \%$ & $99 \%$ \\
\hline kde & 0.000209 & 0.00049 & 0.00127 & 0.00378 & 0.0102 & 0.0589 \\
wkde & 0.000209 & 0.000491 & 0.00126 & 0.00352 & 0.00854 & 0.0527 \\
wkdeA & 0.000209 & 0.00049 & 0.00126 & 0.00354 & 0.00866 & 0.0526 \\
fill+wkde & 0.000205 & 0.00049 & 0.0013 & 0.00416 & 0.0115 & 0.0606 \\
\hline
\end{tabular}

Table 79: Error quantiles evaluating at the observed sample points

\begin{tabular}{lllllll}
\hline & $25 \%$ & $50 \%$ & $75 \%$ & $90 \%$ & $95 \%$ & $99 \%$ \\
\hline kde & 0.0282 & 0.0663 & 0.148 & 0.259 & 0.366 & 2.95 \\
wkde & 0.0233 & 0.0589 & 0.139 & 0.248 & 0.339 & 2.99 \\
wkdeA & 0.0242 & 0.0593 & 0.139 & 0.249 & 0.341 & 3 \\
fill+wkde & 0.0225 & 0.0577 & 0.138 & 0.244 & 0.323 & 2.16 \\
\hline
\end{tabular}


Case No. 40

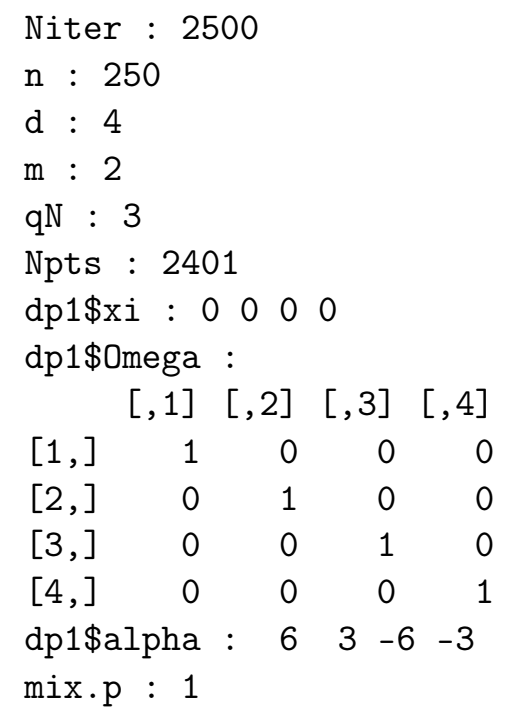

Table 80: Error quantiles for a fixed grid of points

\begin{tabular}{lllllll}
\hline & $25 \%$ & $50 \%$ & $75 \%$ & $90 \%$ & $95 \%$ & $99 \%$ \\
\hline kde & 0.00198 & 0.016 & $4.96 \mathrm{E}+11$ & $3.54 \mathrm{E}+51$ & $3.73 \mathrm{E}+82$ & Inf \\
wkde & 0.00198 & 0.0156 & $4.06 \mathrm{E}+11$ & $2.84 \mathrm{E}+51$ & $2.83 \mathrm{E}+82$ & Inf \\
wkdeA & 0.00198 & 0.0156 & $4.34 \mathrm{E}+11$ & $3.07 \mathrm{E}+51$ & $3.15 \mathrm{E}+82$ & Inf \\
fill+wkde & 0.00243 & 0.0278 & $9.96 \mathrm{E}+13$ & $4.85 \mathrm{E}+58$ & $4.97 \mathrm{E}+93$ & Inf \\
\hline
\end{tabular}

Table 81: Error quantiles evaluating at the observed sample points

\begin{tabular}{lllllll}
\hline & $25 \%$ & $50 \%$ & $75 \%$ & $90 \%$ & $95 \%$ & $99 \%$ \\
\hline kde & 0.0264 & 0.0573 & 0.11 & 0.197 & 0.292 & 0.696 \\
wkde & 0.0251 & 0.0554 & 0.107 & 0.193 & 0.284 & 0.679 \\
wkdeA & 0.0257 & 0.0559 & 0.106 & 0.189 & 0.278 & 0.663 \\
fill+wkde & 0.0201 & 0.0435 & 0.0803 & 0.142 & 0.214 & 0.509 \\
\hline
\end{tabular}


Case No. 41

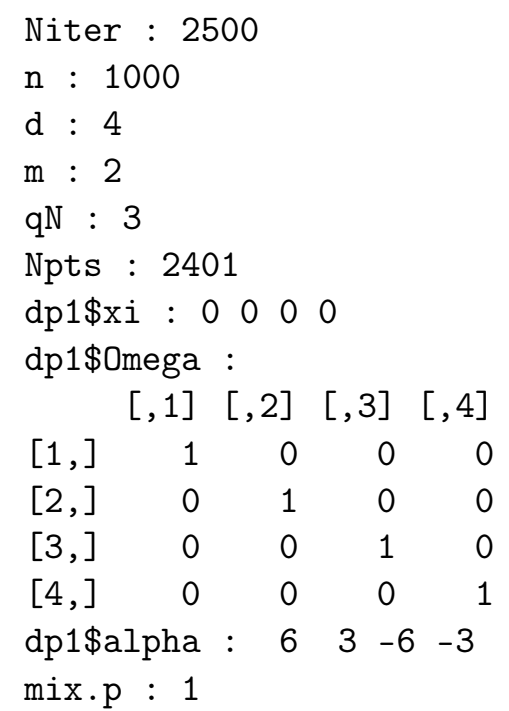

Table 82: Error quantiles for a fixed grid of points

\begin{tabular}{lllllll}
\hline & $25 \%$ & $50 \%$ & $75 \%$ & $90 \%$ & $95 \%$ & $99 \%$ \\
\hline kde & 0.00189 & 0.0135 & $3.41 \mathrm{E}+10$ & $2.95 \mathrm{E}+47$ & $1.92 \mathrm{E}+76$ & Inf \\
wkde & 0.0019 & 0.013 & $2.43 \mathrm{E}+10$ & $2.13 \mathrm{E}+47$ & $1.38 \mathrm{E}+76$ & Inf \\
wkdeA & 0.00189 & 0.013 & $2.58 \mathrm{E}+10$ & $2.27 \mathrm{E}+47$ & $1.45 \mathrm{E}+76$ & Inf \\
fill+wkde & 0.00348 & 0.0329 & $1.26 \mathrm{E}+14$ & $5.02 \mathrm{E}+58$ & $4.16 \mathrm{E}+93$ & Inf \\
\hline
\end{tabular}

Table 83: Error quantiles evaluating at the observed sample points

\begin{tabular}{lllllll}
\hline & $25 \%$ & $50 \%$ & $75 \%$ & $90 \%$ & $95 \%$ & $99 \%$ \\
\hline kde & 0.0154 & 0.0332 & 0.0604 & 0.102 & 0.148 & 0.351 \\
wkde & 0.015 & 0.0327 & 0.0599 & 0.0983 & 0.138 & 0.316 \\
wkdeA & 0.015 & 0.0324 & 0.0581 & 0.0946 & 0.134 & 0.311 \\
fill+wkde & 0.0128 & 0.028 & 0.0517 & 0.0801 & 0.105 & 0.235 \\
\hline
\end{tabular}




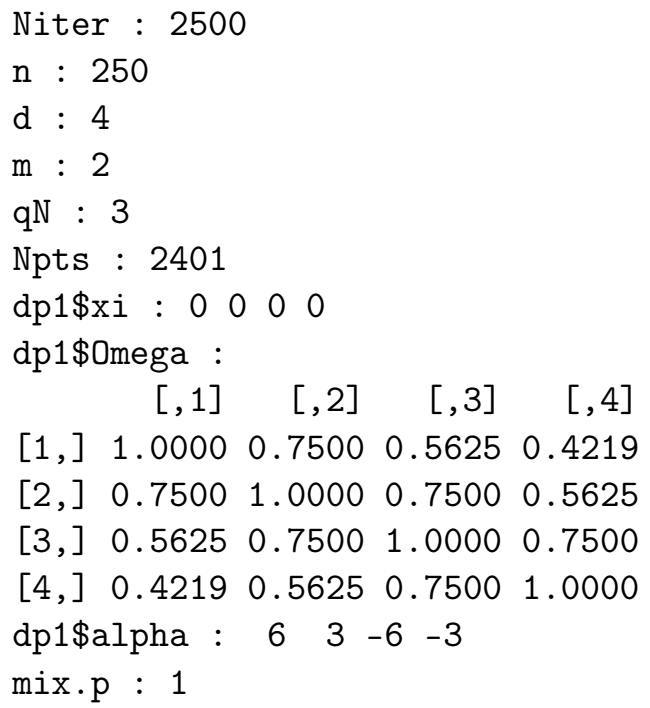

Table 84: Error quantiles for a fixed grid of points

\begin{tabular}{lllllll}
\hline & $25 \%$ & $50 \%$ & $75 \%$ & $90 \%$ & $95 \%$ & $99 \%$ \\
\hline kde & $1.51 \mathrm{E}-06$ & 0.00317 & $6.56 \mathrm{E}+09$ & $2.19 \mathrm{E}+49$ & $9.61 \mathrm{E}+79$ & Inf \\
wkde & $1.3 \mathrm{E}-06$ & 0.00267 & $4.83 \mathrm{E}+09$ & $1.74 \mathrm{E}+49$ & $7.73 \mathrm{E}+79$ & Inf \\
wkdeA & $1.34 \mathrm{E}-06$ & 0.00279 & $5.23 \mathrm{E}+09$ & $1.84 \mathrm{E}+49$ & $7.78 \mathrm{E}+79$ & Inf \\
fill + wkde & 0.0011 & 0.096 & $3.54 \mathrm{E}+13$ & $5.81 \mathrm{E}+57$ & $1.99 \mathrm{E}+93$ & Inf \\
\hline
\end{tabular}

Table 85: Error quantiles evaluating at the observed sample points

\begin{tabular}{lllllll}
\hline & $25 \%$ & $50 \%$ & $75 \%$ & $90 \%$ & $95 \%$ & $99 \%$ \\
\hline kde & 0.0366 & 0.078 & 0.138 & 0.224 & 0.323 & 0.739 \\
wkde & 0.0334 & 0.0718 & 0.129 & 0.21 & 0.302 & 0.693 \\
wkdeA & 0.0335 & 0.0717 & 0.128 & 0.207 & 0.297 & 0.68 \\
fill+wkde & 0.033 & 0.0707 & 0.125 & 0.194 & 0.271 & 0.627 \\
\hline
\end{tabular}




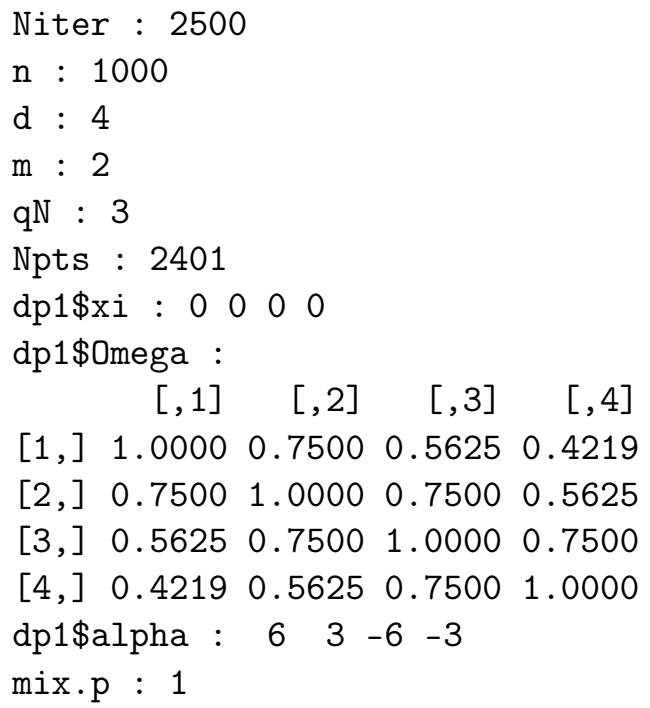

Table 86: Error quantiles for a fixed grid of points

\begin{tabular}{lllllll}
\hline & $25 \%$ & $50 \%$ & $75 \%$ & $90 \%$ & $95 \%$ & $99 \%$ \\
\hline kde & $2.78 \mathrm{E}-07$ & 0.000777 & $5.58 \mathrm{E}+04$ & $1.12 \mathrm{E}+40$ & $1.22 \mathrm{E}+67$ & Inf \\
wkde & $2.53 \mathrm{E}-07$ & 0.000672 & $3 \mathrm{E}+04$ & $6.66 \mathrm{E}+39$ & $1.09 \mathrm{E}+67$ & Inf \\
wkdeA & $2.57 \mathrm{E}-07$ & 0.000686 & $3.3 \mathrm{E}+04$ & $7.05 \mathrm{E}+39$ & $1.12 \mathrm{E}+67$ & Inf \\
fill+wkde & 0.000119 & 0.027 & $1.64 \mathrm{E}+11$ & $4.85 \mathrm{E}+53$ & $1.44 \mathrm{E}+87$ & Inf \\
\hline
\end{tabular}

Table 87: Error quantiles evaluating at the observed sample points

\begin{tabular}{lllllll}
\hline & $25 \%$ & $50 \%$ & $75 \%$ & $90 \%$ & $95 \%$ & $99 \%$ \\
\hline kde & 0.0264 & 0.0563 & 0.0991 & 0.153 & 0.207 & 0.462 \\
wkde & 0.0225 & 0.0487 & 0.0877 & 0.137 & 0.185 & 0.417 \\
wkdeA & 0.0222 & 0.048 & 0.0858 & 0.134 & 0.181 & 0.41 \\
fill+wkde & 0.0228 & 0.0494 & 0.0887 & 0.135 & 0.172 & 0.373 \\
\hline
\end{tabular}




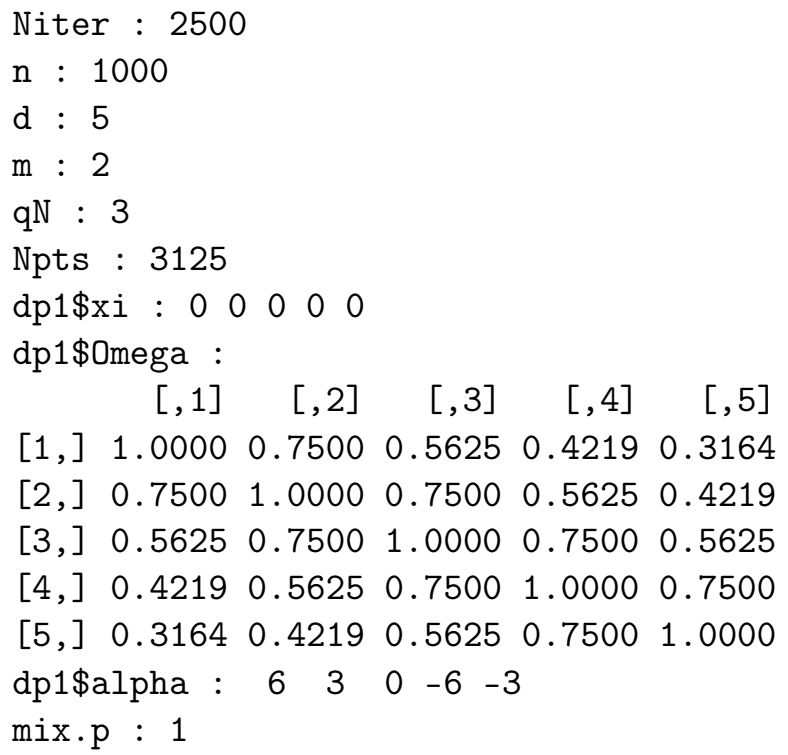

Table 88: Error quantiles for a fixed grid of points

\begin{tabular}{lllllll}
\hline & $25 \%$ & $50 \%$ & $75 \%$ & $90 \%$ & $95 \%$ & $99 \%$ \\
\hline kde & $1.79 \mathrm{E}-10$ & $5.72 \mathrm{E}-06$ & $1.17 \mathrm{E}+04$ & $4.81 \mathrm{E}+46$ & $3.23 \mathrm{E}+78$ & Inf \\
wkde & $1.46 \mathrm{E}-10$ & $5.02 \mathrm{E}-06$ & $4.71 \mathrm{E}+03$ & $1.16 \mathrm{E}+46$ & $1.74 \mathrm{E}+78$ & Inf \\
wkdeA & $1.53 \mathrm{E}-10$ & $5.17 \mathrm{E}-06$ & $5.95 \mathrm{E}+03$ & $1.59 \mathrm{E}+46$ & $1.96 \mathrm{E}+78$ & Inf \\
fill + wkde & 0.000183 & 0.0602 & $3.59 \mathrm{E}+14$ & $3.77 \mathrm{E}+67$ & $6.75 \mathrm{E}+99$ & Inf \\
\hline
\end{tabular}

Table 89: Error quantiles evaluating at the observed sample points

\begin{tabular}{lllllll}
\hline & $25 \%$ & $50 \%$ & $75 \%$ & $90 \%$ & $95 \%$ & $99 \%$ \\
\hline kde & 0.0267 & 0.0577 & 0.106 & 0.188 & 0.287 & 0.73 \\
wkde & 0.0203 & 0.0455 & 0.0883 & 0.163 & 0.254 & 0.667 \\
wkdeA & 0.0208 & 0.0459 & 0.0875 & 0.161 & 0.252 & 0.66 \\
fill+wkde & 0.0196 & 0.0433 & 0.08 & 0.13 & 0.187 & 0.501 \\
\hline
\end{tabular}




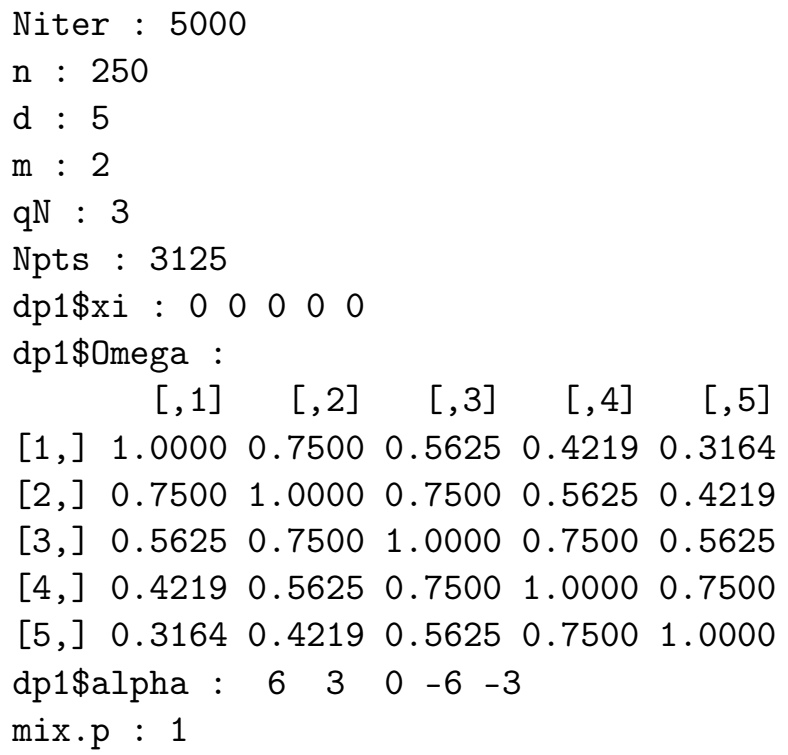

Table 90: Error quantiles for a fixed grid of points

\begin{tabular}{lllllll}
\hline & $25 \%$ & $50 \%$ & $75 \%$ & $90 \%$ & $95 \%$ & $99 \%$ \\
\hline kde & $3.71 \mathrm{E}-09$ & $8.17 \mathrm{E}-05$ & $1.11 \mathrm{E}+10$ & $2.47 \mathrm{E}+58$ & $1.55 \mathrm{E}+89$ & Inf \\
wkde & $2.76 \mathrm{E}-09$ & $6.56 \mathrm{E}-05$ & $6.99 \mathrm{E}+09$ & $1.34 \mathrm{E}+58$ & $1.15 \mathrm{E}+89$ & Inf \\
wkdeA & $3.07 \mathrm{E}-09$ & $7.12 \mathrm{E}-05$ & $8.5 \mathrm{E}+09$ & $1.7 \mathrm{E}+58$ & $1.22 \mathrm{E}+89$ & Inf \\
fill + wkde & 0.0014 & 0.695 & $1.19 \mathrm{E}+17$ & $6.06 \mathrm{E}+71$ & $1.77 \mathrm{E}+105$ & Inf \\
\hline
\end{tabular}

Table 91: Error quantiles evaluating at the observed sample points

\begin{tabular}{lllllll}
\hline & $25 \%$ & $50 \%$ & $75 \%$ & $90 \%$ & $95 \%$ & $99 \%$ \\
\hline kde & 0.0361 & 0.0784 & 0.15 & 0.284 & 0.436 & 1.11 \\
wkde & 0.0314 & 0.0692 & 0.135 & 0.263 & 0.41 & 1.06 \\
wkdeA & 0.0326 & 0.0713 & 0.137 & 0.263 & 0.408 & 1.05 \\
fill+wkde & 0.0291 & 0.0632 & 0.116 & 0.206 & 0.324 & 0.838 \\
\hline
\end{tabular}


Case No. 46

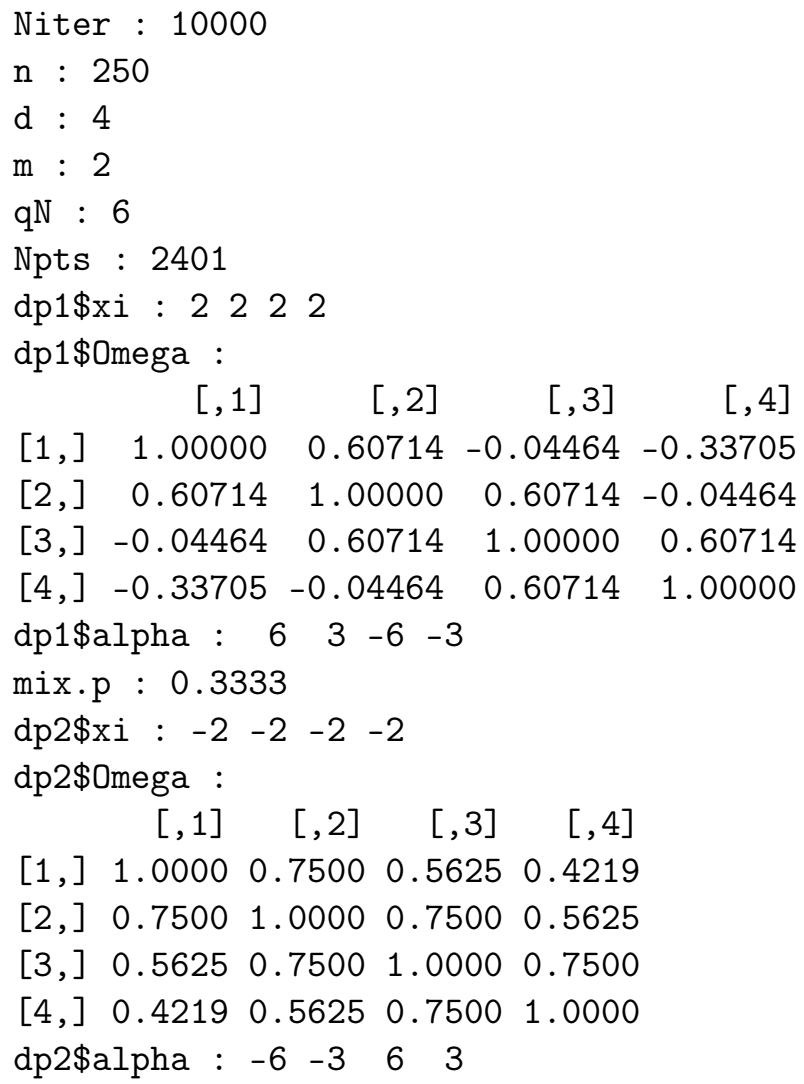

Table 92: Error quantiles for a fixed grid of points

\begin{tabular}{lllllll}
\hline & $25 \%$ & $50 \%$ & $75 \%$ & $90 \%$ & $95 \%$ & $99 \%$ \\
\hline kde & $5.63 \mathrm{E}-14$ & $4.35 \mathrm{E}-07$ & 0.112 & $3.97 \mathrm{E}+11$ & $1.62 \mathrm{E}+25$ & $1.01 \mathrm{E}+55$ \\
wkde & $5.57 \mathrm{E}-14$ & $4.32 \mathrm{E}-07$ & 0.11 & $3.82 \mathrm{E}+11$ & $1.33 \mathrm{E}+25$ & $1.01 \mathrm{E}+55$ \\
wkdeA & $5.55 \mathrm{E}-14$ & $4.29 \mathrm{E}-07$ & 0.11 & $3.82 \mathrm{E}+11$ & $1.51 \mathrm{E}+25$ & $9.41 \mathrm{E}+54$ \\
fill+wkde & $6.93 \mathrm{E}-11$ & 0.000103 & $3.38 \mathrm{E}+03$ & $1.09 \mathrm{E}+19$ & $2.37 \mathrm{E}+37$ & $2.28 \mathrm{E}+73$ \\
\hline
\end{tabular}

Table 93: Error quantiles evaluating at the observed sample points

\begin{tabular}{lllllll}
\hline & $25 \%$ & $50 \%$ & $75 \%$ & $90 \%$ & $95 \%$ & $99 \%$ \\
\hline kde & 0.0307 & 0.0652 & 0.112 & 0.157 & 0.185 & 0.26 \\
wkde & 0.0307 & 0.0651 & 0.112 & 0.157 & 0.185 & 0.261 \\
wkdeA & 0.0305 & 0.0648 & 0.111 & 0.156 & 0.184 & 0.258 \\
fill+wkde & 0.0308 & 0.0654 & 0.112 & 0.158 & 0.185 & 0.26 \\
\hline
\end{tabular}




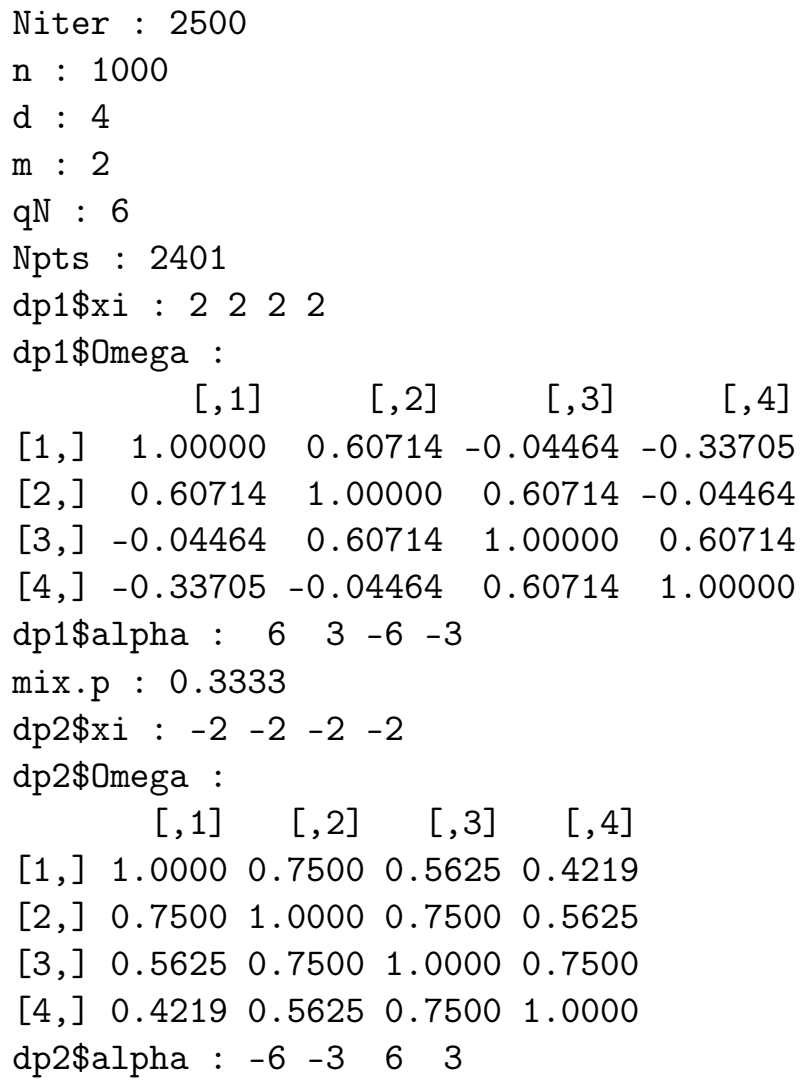

Table 94: Error quantiles for a fixed grid of points

\begin{tabular}{lllllll}
\hline & $25 \%$ & $50 \%$ & $75 \%$ & $90 \%$ & $95 \%$ & $99 \%$ \\
\hline kde & $1.73 \mathrm{E}-25$ & $6.07 \mathrm{E}-15$ & $1.58 \mathrm{E}-07$ & 0.0214 & 160 & $2.42 \mathrm{E}+15$ \\
wkde & $1.66 \mathrm{E}-25$ & $5.72 \mathrm{E}-15$ & $1.5 \mathrm{E}-07$ & 0.0201 & 145 & $8.08 \mathrm{E}+14$ \\
wkdeA & $1.68 \mathrm{E}-25$ & $5.84 \mathrm{E}-15$ & $1.53 \mathrm{E}-07$ & 0.0206 & 150 & $1.44 \mathrm{E}+15$ \\
fill+wkde & $7.64 \mathrm{E}-20$ & $7.15 \mathrm{E}-11$ & 0.00147 & $2.77 \mathrm{E}+08$ & $5.19 \mathrm{E}+21$ & $1.24 \mathrm{E}+49$ \\
\hline
\end{tabular}

Table 95: Error quantiles evaluating at the observed sample points

\begin{tabular}{lllllll}
\hline & $25 \%$ & $50 \%$ & $75 \%$ & $90 \%$ & $95 \%$ & $99 \%$ \\
\hline kde & 0.0229 & 0.0492 & 0.0857 & 0.124 & 0.148 & 0.209 \\
wkde & 0.0225 & 0.0483 & 0.0844 & 0.122 & 0.146 & 0.207 \\
wkdeA & 0.0226 & 0.0485 & 0.0846 & 0.122 & 0.146 & 0.205 \\
fill+wkde & 0.0226 & 0.0486 & 0.085 & 0.123 & 0.147 & 0.206 \\
\hline
\end{tabular}


Case No. 48

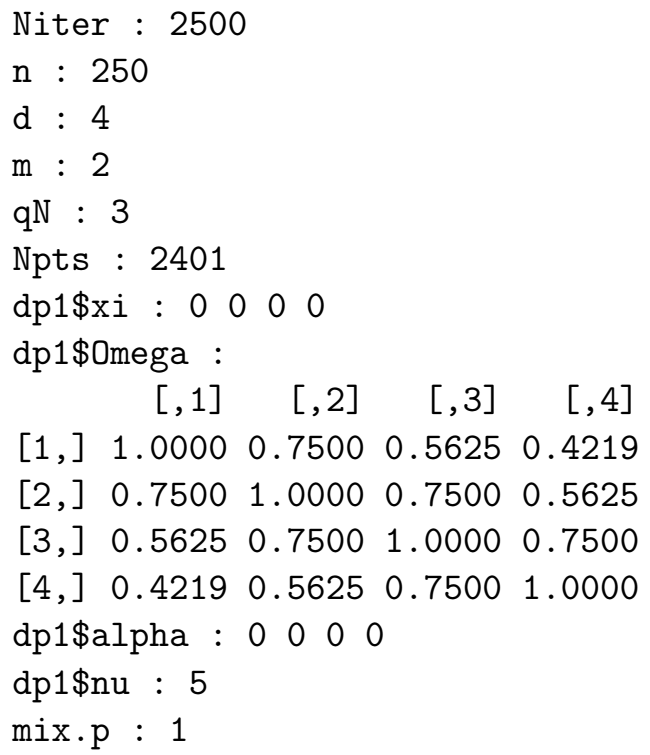

Table 96: Error quantiles for a fixed grid of points

\begin{tabular}{lllllll}
\hline & $25 \%$ & $50 \%$ & $75 \%$ & $90 \%$ & $95 \%$ & $99 \%$ \\
\hline kde & 0.000881 & 0.00218 & 0.00624 & 0.02 & 0.0424 & 0.123 \\
wkde & 0.000897 & 0.00221 & 0.00587 & 0.016 & 0.0319 & 0.1 \\
wkdeA & 0.000893 & 0.0022 & 0.00586 & 0.0163 & 0.0325 & 0.0983 \\
fill+wkde & 0.000826 & 0.00265 & 0.0106 & 0.037 & 0.0715 & 0.199 \\
\hline
\end{tabular}

Table 97: Error quantiles evaluating at the observed sample points

\begin{tabular}{lllllll}
\hline & $25 \%$ & $50 \%$ & $75 \%$ & $90 \%$ & $95 \%$ & $99 \%$ \\
\hline kde & 0.0358 & 0.0763 & 0.137 & 0.25 & 0.461 & 2.11 \\
wkde & 0.0286 & 0.0629 & 0.118 & 0.221 & 0.42 & 2.09 \\
wkdeA & 0.0303 & 0.0647 & 0.118 & 0.22 & 0.421 & 2.09 \\
fill+wkde & 0.0259 & 0.0575 & 0.109 & 0.181 & 0.295 & 1.37 \\
\hline
\end{tabular}




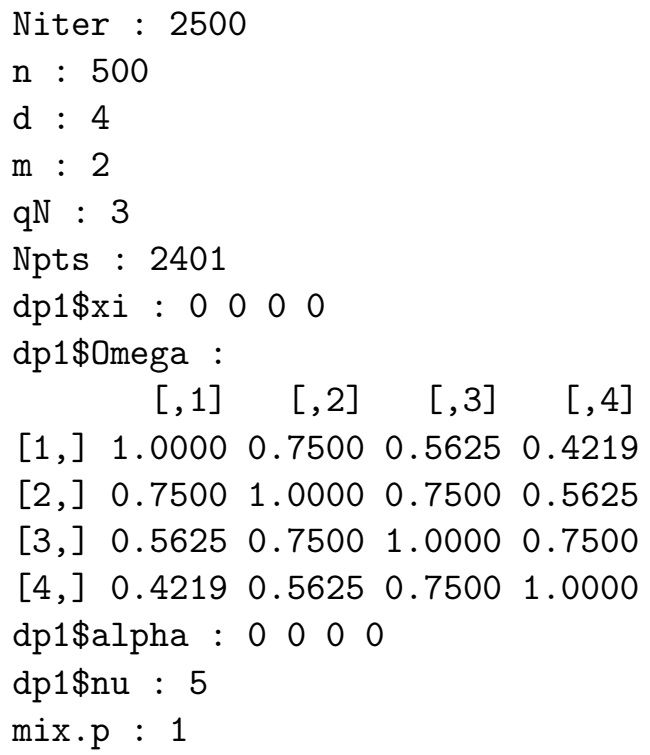

Table 98: Error quantiles for a fixed grid of points

\begin{tabular}{lllllll}
\hline & $25 \%$ & $50 \%$ & $75 \%$ & $90 \%$ & $95 \%$ & $99 \%$ \\
\hline kde & 0.00089 & 0.00221 & 0.00612 & 0.018 & 0.0374 & 0.108 \\
wkde & 0.000908 & 0.00227 & 0.00588 & 0.0141 & 0.0257 & 0.0839 \\
wkdeA & 0.000906 & 0.00226 & 0.00583 & 0.0141 & 0.0257 & 0.0815 \\
fill+wkde & 0.000833 & 0.00229 & 0.00728 & 0.0231 & 0.0462 & 0.135 \\
\hline
\end{tabular}

Table 99: Error quantiles evaluating at the observed sample points

\begin{tabular}{lllllll}
\hline & $25 \%$ & $50 \%$ & $75 \%$ & $90 \%$ & $95 \%$ & $99 \%$ \\
\hline kde & 0.0305 & 0.0651 & 0.116 & 0.204 & 0.375 & 1.62 \\
wkde & 0.0217 & 0.0495 & 0.0956 & 0.178 & 0.342 & 1.73 \\
wkdeA & 0.0226 & 0.0501 & 0.0947 & 0.175 & 0.34 & 1.72 \\
fill+wkde & 0.02 & 0.0452 & 0.0878 & 0.148 & 0.215 & 0.992 \\
\hline
\end{tabular}




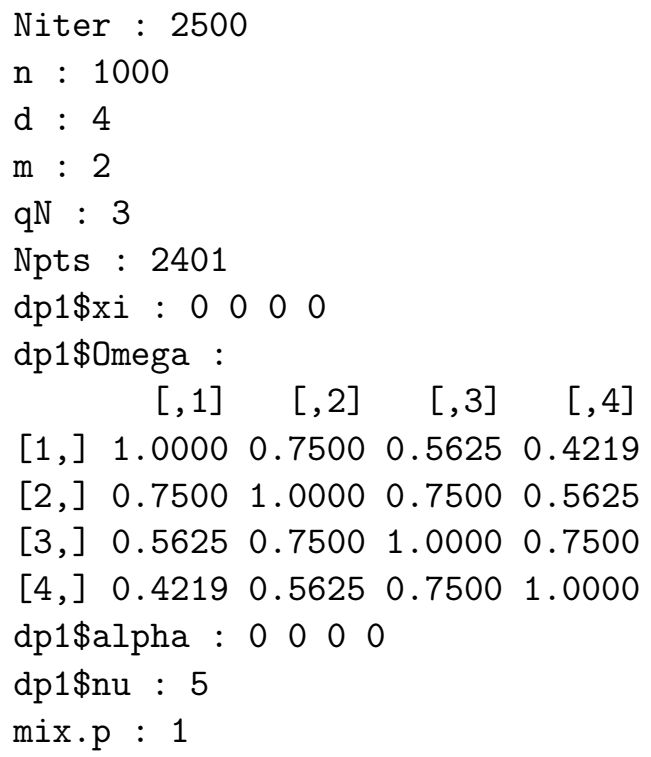

Table 100: Error quantiles for a fixed grid of points

\begin{tabular}{lllllll}
\hline & $25 \%$ & $50 \%$ & $75 \%$ & $90 \%$ & $95 \%$ & $99 \%$ \\
\hline kde & 0.000894 & 0.00223 & 0.00603 & 0.0166 & 0.0333 & 0.0951 \\
wkde & 0.000914 & 0.00232 & 0.00601 & 0.0134 & 0.0224 & 0.0737 \\
wkdeA & 0.000912 & 0.0023 & 0.00594 & 0.0132 & 0.0222 & 0.0713 \\
fill+wkde & 0.000863 & 0.00212 & 0.00569 & 0.014 & 0.0235 & 0.059 \\
\hline
\end{tabular}

Table 101: Error quantiles evaluating at the observed sample points

\begin{tabular}{lllllll}
\hline & $25 \%$ & $50 \%$ & $75 \%$ & $90 \%$ & $95 \%$ & $99 \%$ \\
\hline kde & 0.0259 & 0.0549 & 0.0978 & 0.167 & 0.3 & 1.33 \\
wkde & 0.0166 & 0.0392 & 0.0786 & 0.148 & 0.285 & 1.52 \\
wkdeA & 0.0168 & 0.0391 & 0.0773 & 0.145 & 0.282 & 1.5 \\
fill+wkde & 0.0157 & 0.0361 & 0.0714 & 0.123 & 0.166 & 0.733 \\
\hline
\end{tabular}




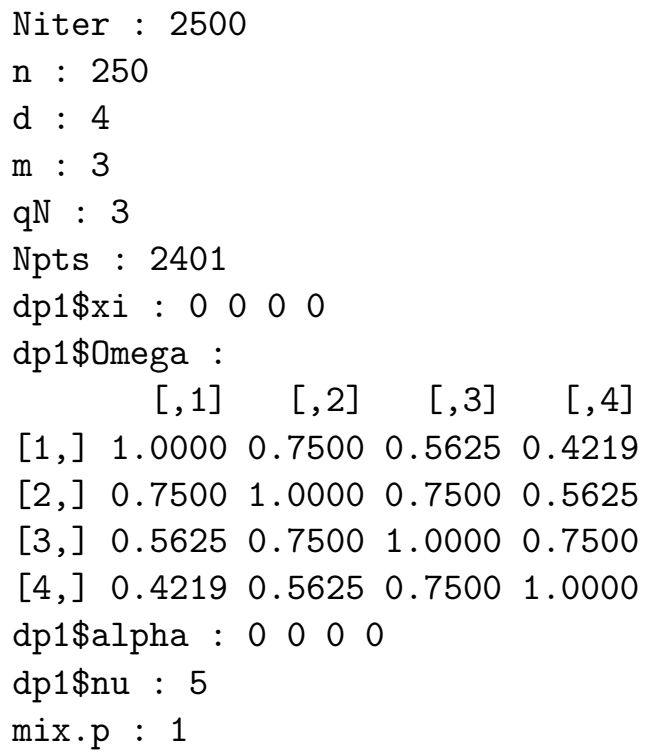

Table 102: Error quantiles for a fixed grid of points

\begin{tabular}{lllllll}
\hline & $25 \%$ & $50 \%$ & $75 \%$ & $90 \%$ & $95 \%$ & $99 \%$ \\
\hline kde & 0.000882 & 0.00219 & 0.00623 & 0.0199 & 0.0421 & 0.123 \\
wkde & 0.000882 & 0.00219 & 0.00623 & 0.0199 & 0.0422 & 0.124 \\
wkdeA & 0.000882 & 0.00219 & 0.00623 & 0.0199 & 0.0421 & 0.124 \\
fill+wkde & 0.000866 & 0.00215 & 0.00634 & 0.021 & 0.0436 & 0.125 \\
\hline
\end{tabular}

Table 103: Error quantiles evaluating at the observed sample points

\begin{tabular}{lllllll}
\hline & $25 \%$ & $50 \%$ & $75 \%$ & $90 \%$ & $95 \%$ & $99 \%$ \\
\hline kde & 0.0358 & 0.0763 & 0.137 & 0.251 & 0.46 & 2.08 \\
wkde & 0.0357 & 0.0761 & 0.137 & 0.254 & 0.467 & 2.13 \\
wkdeA & 0.0358 & 0.0762 & 0.137 & 0.253 & 0.465 & 2.12 \\
fill+wkde & 0.0353 & 0.0753 & 0.136 & 0.248 & 0.455 & 2.07 \\
\hline
\end{tabular}


Case No. 52

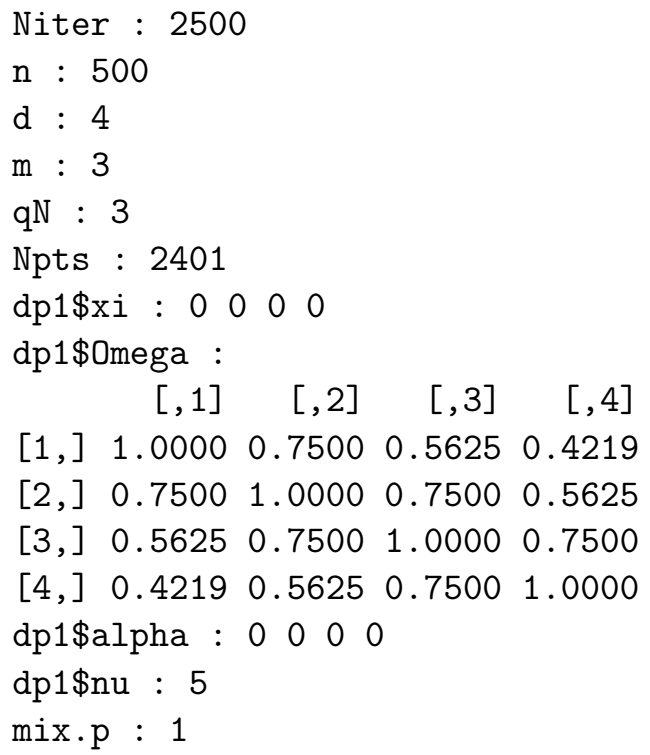

Table 104: Error quantiles for a fixed grid of points

\begin{tabular}{lllllll}
\hline & $25 \%$ & $50 \%$ & $75 \%$ & $90 \%$ & $95 \%$ & $99 \%$ \\
\hline kde & 0.00089 & 0.00221 & 0.00612 & 0.0181 & 0.0374 & 0.108 \\
wkde & 0.00089 & 0.00221 & 0.00612 & 0.0181 & 0.0378 & 0.111 \\
wkdeA & 0.00089 & 0.00221 & 0.00612 & 0.0181 & 0.0377 & 0.11 \\
fill+wkde & 0.000883 & 0.00219 & 0.00613 & 0.0186 & 0.0382 & 0.11 \\
\hline
\end{tabular}

Table 105: Error quantiles evaluating at the observed sample points

\begin{tabular}{lllllll}
\hline & $25 \%$ & $50 \%$ & $75 \%$ & $90 \%$ & $95 \%$ & $99 \%$ \\
\hline kde & 0.0307 & 0.0652 & 0.116 & 0.204 & 0.372 & 1.64 \\
wkde & 0.0305 & 0.0652 & 0.117 & 0.209 & 0.384 & 1.71 \\
wkdeA & 0.0306 & 0.0651 & 0.117 & 0.208 & 0.382 & 1.7 \\
fill+wkde & 0.03 & 0.0641 & 0.115 & 0.201 & 0.367 & 1.63 \\
\hline
\end{tabular}


Case No. 53

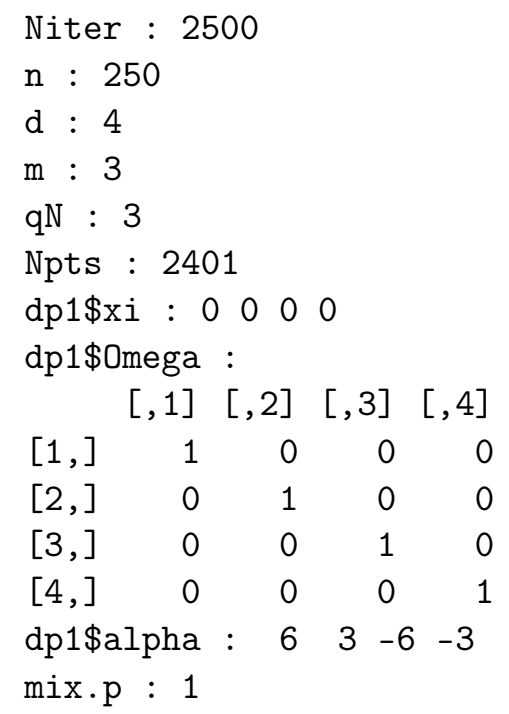

Table 106: Error quantiles for a fixed grid of points

\begin{tabular}{lllllll}
\hline & $25 \%$ & $50 \%$ & $75 \%$ & $90 \%$ & $95 \%$ & $99 \%$ \\
\hline kde & 0.00198 & 0.0159 & $4.86 \mathrm{E}+11$ & $3.75 \mathrm{E}+51$ & $3.97 \mathrm{E}+82$ & Inf \\
wkde & 0.00198 & 0.0159 & $4.72 \mathrm{E}+11$ & $3.57 \mathrm{E}+51$ & $3.75 \mathrm{E}+82$ & Inf \\
wkdeA & 0.00198 & 0.016 & $4.82 \mathrm{E}+11$ & $3.66 \mathrm{E}+51$ & $3.84 \mathrm{E}+82$ & Inf \\
fill+wkde & 0.00204 & 0.0188 & $3.69 \mathrm{E}+12$ & $1.61 \mathrm{E}+56$ & $4.42 \mathrm{E}+90$ & Inf \\
\hline
\end{tabular}

Table 107: Error quantiles evaluating at the observed sample points

\begin{tabular}{lllllll}
\hline & $25 \%$ & $50 \%$ & $75 \%$ & $90 \%$ & $95 \%$ & $99 \%$ \\
\hline kde & 0.0265 & 0.0576 & 0.11 & 0.198 & 0.292 & 0.701 \\
wkde & 0.0265 & 0.0578 & 0.111 & 0.2 & 0.294 & 0.704 \\
wkdeA & 0.0266 & 0.0579 & 0.111 & 0.199 & 0.293 & 0.701 \\
fill+wkde & 0.0241 & 0.0524 & 0.101 & 0.184 & 0.273 & 0.652 \\
\hline
\end{tabular}


Case No. 54

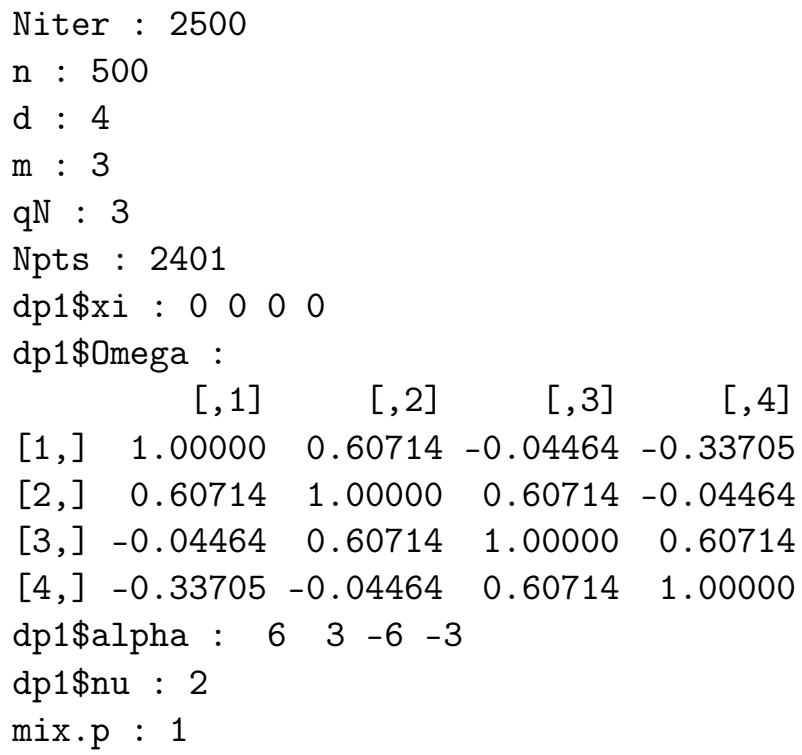

Table 108: Error quantiles for a fixed grid of points

\begin{tabular}{lllllll}
\hline & $25 \%$ & $50 \%$ & $75 \%$ & $90 \%$ & $95 \%$ & $99 \%$ \\
\hline kde & 0.000443 & 0.00242 & 0.0128 & 0.0505 & 0.105 & 0.374 \\
wkde & 0.000443 & 0.00243 & 0.0128 & 0.0506 & 0.106 & 0.373 \\
wkdeA & 0.000443 & 0.00243 & 0.0128 & 0.0506 & 0.106 & 0.373 \\
fill+wkde & 0.000444 & 0.00243 & 0.0128 & 0.0507 & 0.106 & 0.374 \\
\hline
\end{tabular}

Table 109: Error quantiles evaluating at the observed sample points

\begin{tabular}{lllllll}
\hline & $25 \%$ & $50 \%$ & $75 \%$ & $90 \%$ & $95 \%$ & $99 \%$ \\
\hline kde & 0.0348 & 0.085 & 0.189 & 0.344 & 0.556 & 4.94 \\
wkde & 0.035 & 0.0854 & 0.19 & 0.346 & 0.56 & 4.99 \\
wkdeA & 0.035 & 0.0854 & 0.19 & 0.346 & 0.561 & 4.99 \\
fill+wkde & 0.0348 & 0.0849 & 0.189 & 0.344 & 0.555 & 4.94 \\
\hline
\end{tabular}


Summary: relative improvement of error quantiles with Plain variant rel.improvP :

d $\mathrm{m} \operatorname{mix} . \mathrm{p}$

$\begin{array}{llll}1 & 4 & 3 & 0.3333\end{array}$

$\begin{array}{llll}2 & 4 & 2 & 0.3333\end{array}$

$\begin{array}{llll}3 & 4 & 3 & 0.3333\end{array}$

$\begin{array}{llll}4 & 4 & 2 & 0.3333\end{array}$

$\begin{array}{llll}5 & 4 & 3 & 0.3333\end{array}$

$\begin{array}{lllll}6 & 4 & 2 & 0.3333\end{array}$

$\begin{array}{llll}7 & 4 & 3 & 0.6667\end{array}$

$\begin{array}{llll}8 & 4 & 2 & 0.6667\end{array}$

$943 \quad 3 \quad 1.0000$

10421.0000

$\begin{array}{llll}11 & 4 & 3 & 1.0000\end{array}$

12431.0000

13421.0000

14431.0000

15421.0000

16431.0000

$\begin{array}{llll}174 & 2 & 1.0000\end{array}$

$\begin{array}{llll}18 & 3 & 2 & 1.0000\end{array}$

19321.0000

20321.0000

$\begin{array}{llll}21 & 3 & 2 & 1.0000\end{array}$

22321.0000

23321.0000

24321.0000

$\begin{array}{llll}25 & 5 & 2 & 1.0000\end{array}$

$\begin{array}{llll}26 & 5 & 3 & 1.0000\end{array}$

$\begin{array}{llll}27 & 5 & 2 & 0.6667\end{array}$

$\begin{array}{llll}28 & 5 & 3 & 0.6667\end{array}$

$\begin{array}{llll}29 & 5 & 2 & 0.6667\end{array}$

$\begin{array}{llll}30 & 5 & 3 & 0.6667\end{array}$

$\begin{array}{llll}31 & 5 & 2 & 0.6667\end{array}$

n grid.50

obs. 50

grid. 75

obs. 75

grid. 95

obs. 95

$500-7.024 e-06$

0.0062091

$6.057 e-05$

0.0035047

0.0014075

0.0023555

$\begin{array}{lll}500 & -4.816 e-04 & 0.0632107\end{array}$

$4.517 \mathrm{e}-04 \quad 0.0496820$

0.0281589

0.0487456

$8.854 \mathrm{e}-04 \quad 0.0010320$

$4.857 \mathrm{e}-03 \quad 0.0007320$

0.0064476

0.0001832

$\begin{array}{lllll}500 & 3.086 \mathrm{e}-02 & 0.0075780 & 5.299 \mathrm{e}-02 & 0.0066434\end{array}$

0.0596355

0.0062920

$5009.247 e-05-0.0019726-5.787 e-04-0.0034900$

$0.0056127-0.0049934$

$500-1.073 e-02 \quad 0.1587915-8.131 e-03 \quad 0.0766432$

0.16583890 .0535351

$5009.290 \mathrm{e}-05-0.0025718-4.604 \mathrm{e}-04-0.0024352$

$-0.0039162-0.0043010$

$500-1.272 e-02 \quad 0.1035375-6.344 e-03 \quad 0.0723508$

$500-5.496 e-04-0.0061320-2.988 e-03-0.0078146$

$0.1285367 \quad 0.0483129$

$\begin{array}{lllll}500 & -7.959 e-03 & 0.0138169 & 1.543 e-02 & 0.0400199\end{array}$

$0.0067139-0.0068770$

$500-1.523 e-02-0.0115403 \quad 4.932 e-03-0.0129228$

0.1119632

$\begin{array}{llllll}500 & 4.819 e-04 & 0.0001732 & 2.682 e-02 & -0.0115994\end{array}$

0.01990830 .0013560

$\begin{array}{llllll}500 & 4.079 \mathrm{e}-02 & 0.0287409 & 3.354 \mathrm{e}-01 & 0.0208001\end{array}$

$0.1283942-0.0057434$

$500 \quad 1.392 \mathrm{e}-02-0.0005857 \quad 1.794 \mathrm{e}-02-0.0003149$

0.3082178

0.0502174

$\begin{array}{llllll}500 & 1.780 e-01 & 0.0549584 & 1.853 e-01 & 0.0490660\end{array}$

0.08578990 .0052793

$\begin{array}{llllll}500 & 1.549 \mathrm{e}-02 & 0.0183119 & 2.561 \mathrm{e}-02 & 0.0132881\end{array}$

0.3811008

0.0629187

$500 \quad 1.892 \mathrm{e}-01 \quad 0.1097304$

$3.741 \mathrm{e}-010.0922566$

0.0247188

0.0042106

$500 \quad 5.556 \mathrm{e}-02 \quad 0.0653200$

$9.482 \mathrm{e}-02 \quad 0.0619619$

0.3969550

0.0870600

$\begin{array}{lllll}500 & 1.278 \mathrm{e}-01 & 0.0616721 & 1.092 \mathrm{e}-01 & 0.0616103\end{array}$

$\begin{array}{llllll}500 & 7.405 e-02 & 0.0116158 & 5.613 e-02 & 0.0130387\end{array}$

0.3163063

0.0641892

$500 \quad 1.212 \mathrm{e}-01 \quad 0.0649599$

$1.197 \mathrm{e}-01 \quad 0.0632092$

0.1263927

0.0748406

$500-3.033 e-02 \quad 0.1249595$

$2.755 e-02 \quad 0.0842165$

0.1171125

0.0287566

$500 \quad 1.408 \mathrm{e}-02$

0.1458633

$1.316 e-01$

0.1219351

0.0703953

$500 \quad 1.280 \mathrm{e}-02 \quad 0.1119781$

$5.674 \mathrm{e}-02$

0.1193372

0.0500413

0.0997428

0.1957737

0.0798755

$\begin{array}{lllll}500 & 2.696 \mathrm{e}-01 & 0.1640928 & 5.246 \mathrm{e}-01 & 0.1346124\end{array}$

$\begin{array}{llllll}500 & 4.909 \mathrm{e}-02 & 0.0370564 & 1.004 \mathrm{e}-01 & 0.0243688\end{array}$

0.1378309

0.0490871

$500-7.520 e-02-0.0304363-2.542 e-02-0.0044766$

$5001.255 e-03-0.0060955 \quad 6.055 e-04-0.0053435$

.3636144

0.0941541

$0.1197060 \quad 0.0004601$

$0.1318902 \quad 0.1014228$

$\begin{array}{lllll}500 & -6.223 e-04 & 0.0587803 & -5.015 e-05 & 0.0519677\end{array}$

$0.0031362-0.0110032$

$\begin{array}{lllll}500 & -6.200 e-05 & 0.0058907 & -1.652 e-05 & 0.0041479\end{array}$

0.0112995

0.0727365

$\begin{array}{lllll}500 & 7.981 \mathrm{e}-02 & 0.0089196 & 8.742 \mathrm{e}-02 & 0.0057118\end{array}$

$0.0007639 \quad 0.0044376$

$\begin{array}{lllll}32 & 5 & 3 & 0.6667\end{array}$

$500 \quad 8.174 \mathrm{e}-03 \quad 0.0005249$

$9.317 e-03$

0.2295232

0.0057754

$\begin{array}{llllll}33 & 5 & 3 & 1.0000 & 100\end{array}$

$\begin{array}{llll}34 & 5 & 3 & 1.0000\end{array}$

$10001.913 e-02$

0.0719979

$1.118 \mathrm{e}-01$

0.0005979

$0.0327445-0.0004954$

$250 \quad 2.444 \mathrm{e}-02 \quad 0.0129532$

$4.412 e-02$

$0.1075626 \quad 0.0061596$

$250-4.661 e-03-0.0003045$

$\begin{array}{lllll}36 & 4 & 2 & 1.0000\end{array}$

$\begin{array}{lll}250 & 1.988 e-03 & 0.0306898\end{array}$

$2.770 e-03$

0.0070907

$0.0300844-0.0081575$

$\begin{array}{llll}37 & 4 & 2 & 1.0000\end{array}$

$000 \quad 1.177 \mathrm{e}-03 \quad 0.0211555$

$2.359 \mathrm{e}-02 \quad 0.0336224$

$0.0155507-0.0066655$

$\begin{array}{llll}38 & 4 & 2 & 0.3333\end{array}$

$250-2.769 e-04$

0.0349276

$5.332 \mathrm{e}-02 \quad 0.0386698$

$0.0848010 \quad 0.0593612$

$0.1650018 \quad 0.1494835$

$\begin{array}{llll}39 & 4 & 2 & 0.3333\end{array}$

$250-1.565 e-03$

0.1122842

$6.568 e-040.0278809$

0.0361631

0.0305796

$\begin{array}{llll}40 & 4 & 2 & 1.0000\end{array}$

250

$2.481 e-02$

0.0342828

$6.472 \mathrm{e}-03 \quad 0.0603346$

0.1604286

0.0743937

41421.00001000

$3.731 \mathrm{e}-02$

$1.827 e-01$

0.0243983

0.2413600

0.0253359

$\begin{array}{llll}42 & 4 & 2 & 1.0000 \quad 250\end{array}$

1. $553 e-01$

0.0142255

$2.865 \mathrm{e}-010.0088516$

0.2804137

0.0682228

43421.00001000

1. $352 e-01$

0.0793144

$2.639 \mathrm{e}-010.0659200$

0.1952579

0.0646610

44521.0000100

$\begin{array}{lllll}45 & 5 & 2 & 1.0000 & 25\end{array}$

$1.213 e-0$

.1350389

$4.624 \mathrm{e}-01 \quad 0.1144852$

0.1098095

0.1037914

$2501.976 \mathrm{e}-01$

0.2117341

$5.973 e-01 \quad 0.1692204$

0.4616741

0.1138866

$3.706 \mathrm{e}-01 \quad 0.0972674$

0.2576898

0.0604635

$\begin{array}{lllllllll}46 & 4 & 2 & 0.3333 & 250 & 7.136 \mathrm{e}-03 & 0.0008641 & 2.205 \mathrm{e}-02 & 0.0008915\end{array}$

$\begin{array}{lllllllll}47 & 4 & 2 & 0.3333 & 1000 & 5.672 \mathrm{e}-02 & 0.0173900 & 5.416 \mathrm{e}-02 & 0.0152593\end{array}$

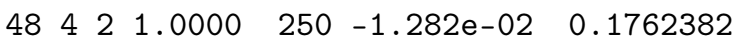

$49 \quad 4 \quad 2 \quad 1.0000 \quad 500-2.638 \mathrm{e}-02$

0.2399888

$5.927 e-02 \quad 0.1358727$

0.1833463

$-0.0002455$

$0.0917034 \quad 0.0135010$

$0.2485566 \quad 0.0901304$

$\begin{array}{llll}3.831 \mathrm{e}-02 & 0.1777767 & 0.3134105 & 0.0873002\end{array}$ $\begin{array}{lllll}50 & 4 & 2 & 1.0000 & 100\end{array}$ $\begin{array}{llll}51 & 4 & 3 & 1.0000\end{array}$

$\begin{array}{llll}52 & 4 & 3 & 1.0000\end{array}$

$53 \quad 4 \quad 3 \quad 1.0000$

0.2860935

$3.176 \mathrm{e}-03 \quad 0.1967130$

0.3255958

0.0511283 $250-5.343 e-04 \quad 0.0023868-1.210 e-04-0.0022015-0.0012113-0.0157336$ $\begin{array}{llllllll}500 & -5.069 e-04 & 0.0008678 & -1.077 e-03 & -0.0087687 & -0.0101262 & -0.0329717\end{array}$ $\begin{array}{llllllll}250 & 7.377 e-05 & -0.0036595 & 2.863 e-02 & -0.0080380 & 0.0559315 & -0.0069071\end{array}$ 54431.0000 $500-8.766 e-04-0.0047209-1.407 e-03-0.0039813-0.0012095-0.0084011$ 
Summary: relative improvement of error quantiles with Fill variant rel.improvf :

d $m$ mix.p

$\begin{array}{llll}1 & 4 & 3 & 0.3333\end{array}$

$\begin{array}{llll}2 & 4 & 2 & 0.3333\end{array}$

$\begin{array}{llll}3 & 4 & 3 & 0.3333\end{array}$

$\begin{array}{llll}4 & 4 & 2 & 0.3333\end{array}$

$\begin{array}{llll}5 & 4 & 3 & 0.3333\end{array}$

$\begin{array}{llll}6 & 4 & 2 & 0.3333\end{array}$

$\begin{array}{llll}7 & 4 & 3 & 0.6667\end{array}$

$\begin{array}{llll}8 & 4 & 2 & 0.6667\end{array}$

$943 \quad 3 \quad 1.0000$

10421.0000

$1143 \quad 31.0000$

12431.0000

13421.0000

14431.0000

15421.0000

16431.0000

$\begin{array}{llll}174 & 2 & 1.0000\end{array}$

$\begin{array}{llll}18 & 3 & 2 & 1.0000\end{array}$

19321.0000

20321.0000

$\begin{array}{llll}21 & 3 & 2 & 1.0000\end{array}$

22321.0000

23321.0000

24321.0000

$\begin{array}{llll}25 & 5 & 2 & 1.0000\end{array}$

$\begin{array}{llll}26 & 5 & 3 & 1.0000\end{array}$

$\begin{array}{llll}27 & 5 & 2 & 0.6667\end{array}$

$\begin{array}{llll}28 & 5 & 3 & 0.6667\end{array}$

$\begin{array}{llll}29 & 5 & 2 & 0.6667\end{array}$

$\begin{array}{llll}30 & 5 & 3 & 0.6667\end{array}$

$\begin{array}{llll}31 & 5 & 2 & 0.6667\end{array}$

$\begin{array}{llll}32 & 5 & 3 & 0.6667\end{array}$

$\begin{array}{llll}33 & 5 & 3 & 1.0000\end{array}$

$\begin{array}{llll}34 & 5 & 3 & 1.0000\end{array}$

$\begin{array}{llll}35 & 4 & 3 & 1.0000\end{array}$

n grid. 50

obs.50 grid.75

obs.75 grid.95

obs. 95

$500-4.600 e-04$

$0.0065579-6.411 e-03$

$2.335 e-03-6.859 e-02$

0.0022439

$500-1.959 e-02$

$0.0649588-6.643 e-02$

$3.934 e-02-1.079 e+00$

0.0440172

$-1.399 e+01$

$0.0004061-9.173 e+00$

$6.291 e-05-6.550 e+06$

$-0.0002471$

$500-1.160 e+04$

$0.0027459-3.888 e+03$

$1.721 e-03-3.067 e+14$

0.0031734

$5009.827 e-04$

$0.0007644 \quad 1.951 \mathrm{e}-05$

$-2.568 e-04-2.826 e-03$

0.0008446

$5006.021 \mathrm{e}-03$

$0.17415477 .287 e-04$

$8.196 \mathrm{e}-02 \quad 6.284 \mathrm{e}-02$

0.1145357

$5008.073 e-04$

$0.00089741 .189 \mathrm{e}-03$

$-2.243 e-04-2.716 e-03$

0.0003218

$500 \quad 1.550 \mathrm{e}-02$

$0.16573292 .504 \mathrm{e}-03$

8.309e-02 4.552e-02

0.1147171

$500 \quad 1.076 \mathrm{e}-03$

$0.00227201 .565 \mathrm{e}-03$

$1.678 e-03 \quad 2.173 e-03$

0.0033365

$500-8.867 e-01$

$500-1.976 e-01$

$0.3168617-1.270 e+00$

$3.677 e-01-6.757 e-01$

0.3515219

$500-2.617 \mathrm{e}-01$

$0.1761395-4.734 e-01$

$1.718 e-01-2.449 e-01$

0.1259928

$500-8.144 e-01$

$0.1002917-5.022 e+01$

$9.682 \mathrm{e}-02-1.329 \mathrm{e}+10$

0.0910333

$2.178 \mathrm{e}-01-1.122 \mathrm{e}+15$

0.2994834

$-2.003 e+02$

$0.0315370-2.827 e+05$

$3.579 e-03-2.836 e+06$

0.0224118

$500-1.805 e+00$

$0.0184925-1.562 e+01$

$5.117 e-02-1.488 e+15$

0.1669600

$500-3.474 e+01$

$1.698 e-02-4.247 e+05$

0.0248494

$500-2.543 e+00$

$0.1123845-2.280 e+06$

$1.026 e-01-2.870 e+17$

0.1781653

$500-2.745 e+00$

$0.0673693-2.094 e+00$

$7.298 e-02-1.039 e+05$

0.1014109

$500-1.717 e+01$

$0444310-1.163 e+00$

$5.524 e-02-4.409 e+00$

0.1023358

$500-6.003 e+00$

$.0085571-5.043 e+02$

$-7.551 e-04-4.679 e+08$

0.0422174

$500 \quad 1.853 e-02$

$0.0447325-1.435 \mathrm{e}+00$

$5.213 e-02-2.967 e+00$

0.0926267

$5002.427 \mathrm{e}-02$

$0.29060525 .808 e-02$

$2.419 e-01 \quad 1.503 e-01$

0.2865112

$500-3.636 e-01$

$0.11151061 .054 \mathrm{e}-02$

$8.790 \mathrm{e}-02 \quad 7.459 \mathrm{e}-02$

0.1522683

$500-7.552 \mathrm{e}+03$

$0.0895953-3.814 e-01$

$4.749 e-02-1.092 e+00$

0.0648791

$500-5.164 \mathrm{e}+01$

$0.2255596-4.661 e+08$

$2.350 e-01-1.918 e+18$

0.3047575

$8.528 e-02-8.533 e+10$

0.0845638

$3.002 e-03-9.525 e-03$

0.1642382

$500-1.229 e-02-0.0049056-4.794 e-03$

$-5.097 e-03-6.760 e-03$

0.0112358

$500-4.957 \mathrm{e}-02$

$0.0695890-1.461 e-01$

$5.281 e-02-2.013 e+00$

0.0838223

$500-7.283 e-03 \quad 0.0087676-2.375 e-02$

$500-1.716 e+09$

$0.0031929-2.377 e+13$

$4.969 e-03-2.364 e-01$

0.0088703

$500-5.526 e+04-0.0007819-2.782 e+06$

$7.005 e-04-1.561 e+23$

0.0033200

$000-9.046 e+01$

$0.1110201-7.476 \mathrm{e}+05$

$0.0447503-6.994 e+02$

$-0.0011976$

$250-1.906 \mathrm{e}+01$

$1.119 e-01-6.829 e+13$

0.1251793

$250-1.403 e-01$

$0.1631826-1.835 \mathrm{e}-01$

$5.600 e-02-1.127 e+09$

0.0475500

0.0918364

$0.3671602-6.925 e-01$

$248 e-01-1.843 e-01$

0.3108264

$000-2.412 e+00$

$0.2654077-1.998 e+00$

. $680 e-01-5.919 e-01$

0.3468042

$0.0378355-6.597 e-02$

.

0.0312826

$250 \quad 3.576 e-04 \quad 0.1308397-2.349 e-02$

$2.369 \mathrm{e}-02-9.244 \mathrm{e}-01$

0.1185749

$0.2406346-1.997 e+02$

$6.312 \mathrm{e}-02-1.320 \mathrm{e}-01$

0.2652797

$\begin{array}{llllll}40 & 4 & 2 & 1.0000 & 250 & -7.377 \mathrm{e}-01\end{array}$

$0.1570527-3.692 e+03$

$2.695 e-01-1.333 e+11$

0.2897565

$0.0927426-5.400 e+03$

$1.433 e-01-2.168 e+17$

0.1600587

$0.1223061-2.937 e+06$

$286 e-02-2.071 e+13$

0.1685462

$0.2498730-3.070 e+10$

0.3463910

$0.1943648-1.067 e+07$

$2.472 e-01-2.087 e+21$

0.2580643

$\begin{array}{llll}45 & 5 & 2 & 1.0000\end{array}$

$250-8.504 e+03$

$-0.0037129-3.010 e+04$

$2.217 e-01-1.141 e+16$

$-0.0025253$

$\begin{array}{llllll}47 & 4 & 2 & 0.3333 & 1000 & -1.179 e+04\end{array}$

$0.0110207-9.264 e+03$

0.0089931

$0.2459490-6.981 e-01$

8. $527 e-03-3.248 e+19$

0.3602301

$\begin{array}{llllll}49 & 4 & 2 & 1.0000 & 500 & -3.605 e-02\end{array}$

$0.3055664-1.901 e-01$

.

0.4245999

$\begin{array}{llllll}50 & 4 & 2 & 1.0000 & 1000 & 4.666 \mathrm{e}-02\end{array}$

$0.34338475 .629 e-02$

$2.454 e-01-2.352 e-01$

0.4479900

$\begin{array}{llll}51 & 4 & 3 & 1.0000\end{array}$

$250 \quad 1.637 \mathrm{e}-02$

$0.0127077-1.676 e-02$

$2.699 e-012.940 e-01$

0.0099689

$\begin{array}{llll}52 & 4 & 3 & 1.0000\end{array}$

$5001.214 \mathrm{e}-02$

$0.0172478-1.972 \mathrm{e}-03$

$9.533 e-03-3.566 e-02$

0.0128858

$\begin{array}{llll}53 & 4 & 3 & 1.0000\end{array}$

$250-1.813 e-01$

$0.0893326-6.598 e+00$

$1.108 e-02-2.081 e-02$

0.0658840

$\begin{array}{lllllll}54 & 4 & 3 & 1.0000 & 500 & -2.634 \mathrm{e}-03\end{array}$

$0.0006553-5.151 e-03$

0.0011444 